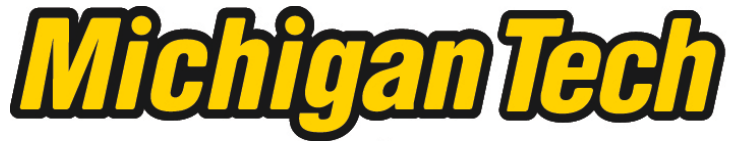 \\ Michigan Technological University Create the Future Digital Commons @ Michigan Tech
}

Dissertations, Master's Theses and Master's Reports - Open

Dissertations, Master's Theses and Master's

Reports

2015

\section{MOLECULAR MODELING OF EPON 862-DETDA / CARBON COMPOSITES}

Cameron Hadden

Michigan Technological University

Follow this and additional works at: https://digitalcommons.mtu.edu/etds

Part of the Mechanical Engineering Commons

Copyright 2015 Cameron Hadden

\section{Recommended Citation}

Hadden, Cameron, "MOLECULAR MODELING OF EPON 862-DETDA / CARBON COMPOSITES",

Dissertation, Michigan Technological University, 2015.

https://doi.org/10.37099/mtu.dc.etds/946

Follow this and additional works at: https://digitalcommons.mtu.edu/etds

Part of the Mechanical Engineering Commons 


\title{
MOLECULAR MODELING OF EPON 862-DETDA / CARBON COMPOSITES
}

\author{
By \\ Cameron Hadden
}

A DISSERTATION

Submitted in partial fulfillment of the requirements for the degree of

\section{DOCTOR OF PHILOSOPHY}

In Mechanical Engineering - Engineering Mechanics

\section{MICHIGAN TECHNOLOGICAL UNIVERSITY}

2015

(c) 2015 Cameron M. Hadden 

This dissertation is hereby approved in partial fulfillment of the requirements for the Degree of DOCTOR OF PHILOSOPHY in Mechanical EngineeringEngineering Mechanics.

Department of Mechanical Engineering - Engineering Mechanics

Dissertation Advisor: $\quad$ Dr. Gregory M. Odegard

Committee Member: Dr. Reza Shahbazian Yassar

Committee Member: Dr. Julia King

Committee Member: Dr. Gowtham

Department Chair: Dr. William Predabon 



\section{Table of Contents}

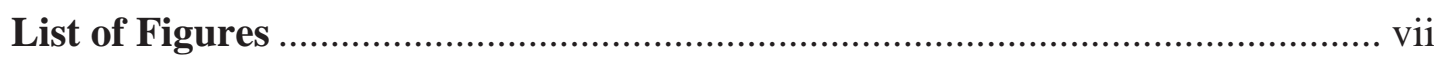

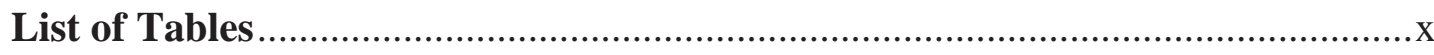

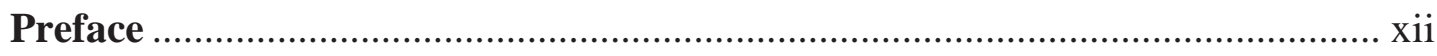

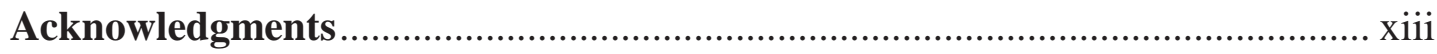

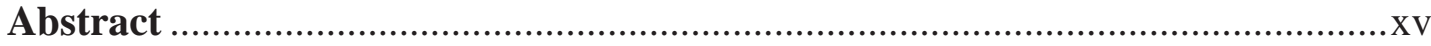

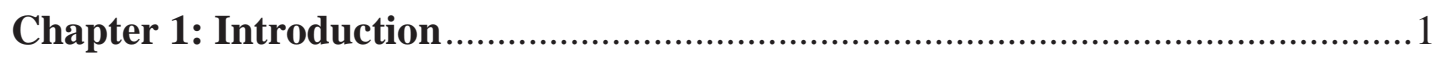

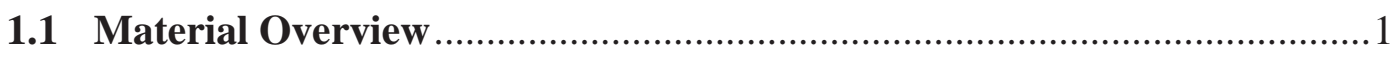

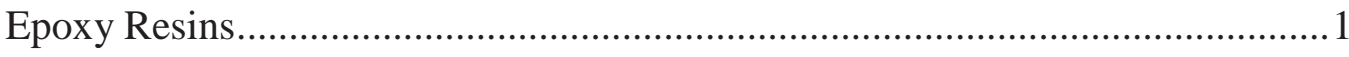

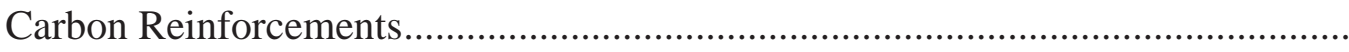

2

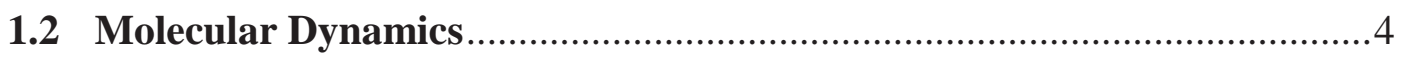

Chapter 2: Molecular Modeling Procedures ......................................................

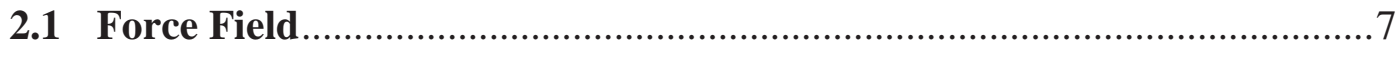

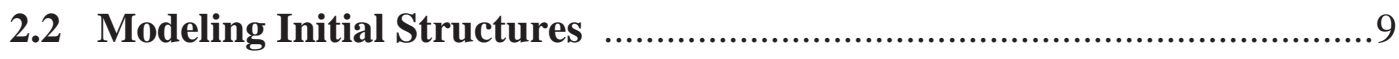

2.3 Crosslinking Procedure ……………………......................................12

Chapter 3: Modeling the Fiber/Matrix Interface ……………...............................16

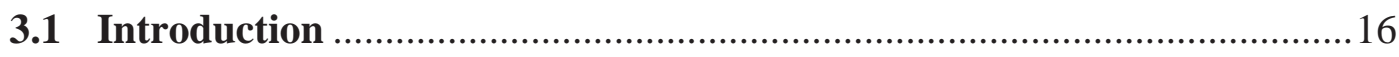

3.2 Modeling Procedure ……………………………................................ 17

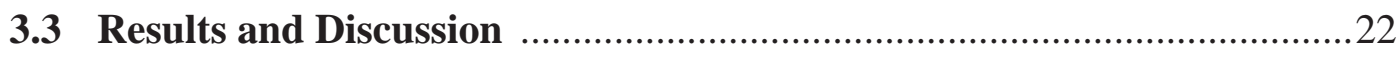


Polymer Mass Density Characteristics ....................................................... 23

Polymer Internal Stress Characteristics ........................................................ 26

Polymer Potential Energy Characteristics ....................................................... 28

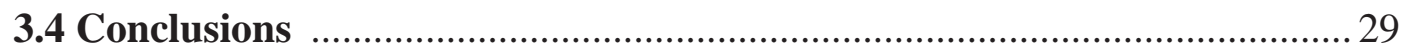

Chapter 4: Multi-Scale Modeling of Graphene Doped Epoxy ........................... 30

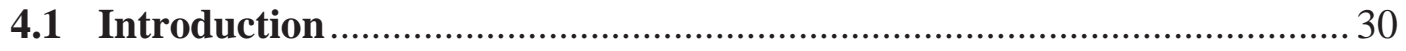

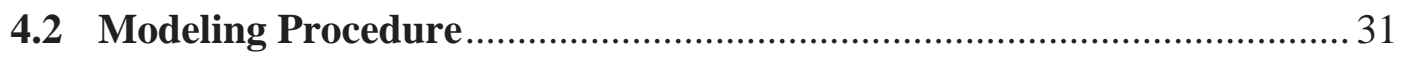

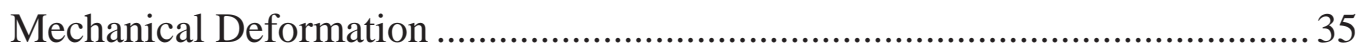

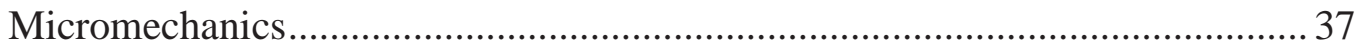

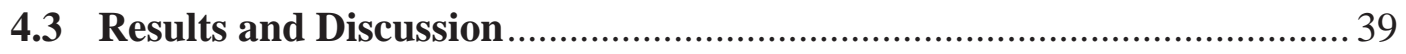

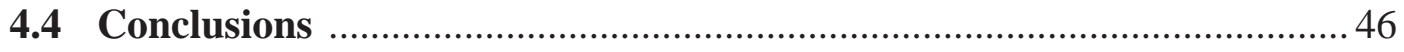

Chapter 5: Multi-Scale Modeling of Graphene Oxide / Epoxy Composites....... 47

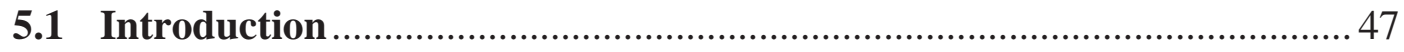

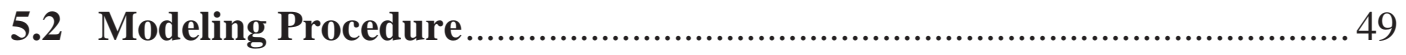

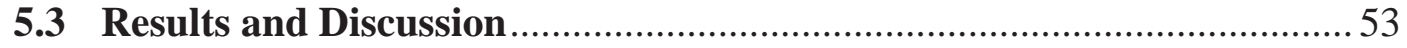

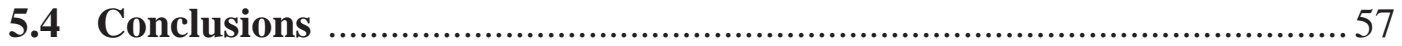

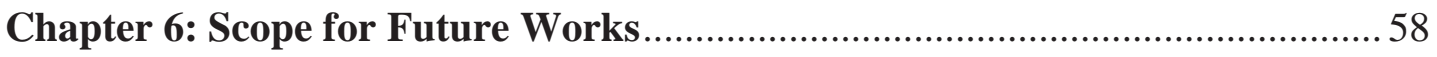

6.1 Improving Modeling Procedures ........................................................ 58

6.2 Modeling Functionalized Graphene and Surface Treatments................59

6.3 Modeling Aging in Composite Structures .......................................... 60 


\section{Appendix A}

\section{LAMMPS scripts for MD simulations, MM simulations and Deformation}

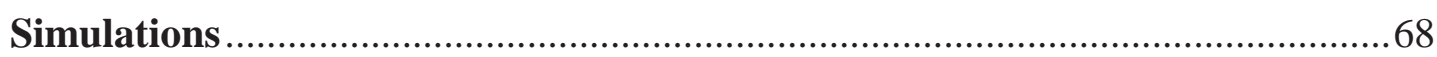

A.1 Molecular Energy Minimization script ...................................................6 68

A.2 MD NVT simulation script for equilibration ............................................69

A.3 MD NPT simulation script for tensile deformation ....................................72

A.4 MD NVT simulation script for shear deformation..................................... 76

A.5 MD OPLS UA parameters from coordinates file..................................... 77

\section{Appendix B}

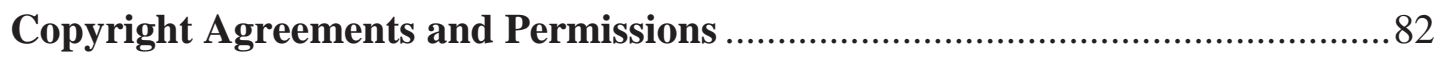

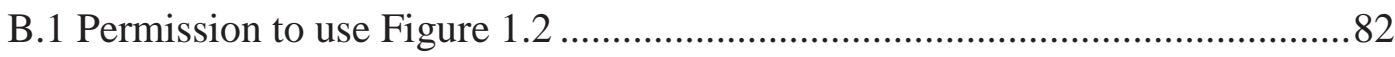

B.2 Copyright agreement for using selected text and Figures in Chapter 2 ..........83 


\section{List of Figures}

Figure 1.1 - Epoxed (a) and Amine (b) moleculuar structures ................................ 2

Figure 1.2 - Predicted internal structure for carbon fibers, surface atom structures,

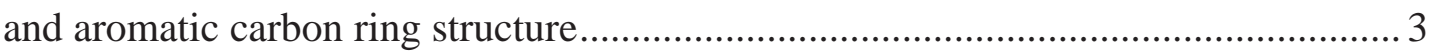

Figure 2.1 - A visual representation of each energy contribution that is considered by

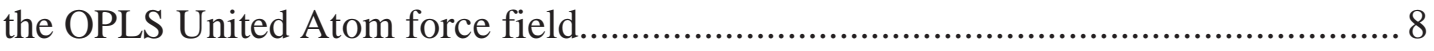

Figure 2.2 - Molecular structures of a) EPON 862 and b) DETDA shown with simulated image

Figure 2.3 - Visual representation of the fully equilibrated 2:1 ratio of EPON

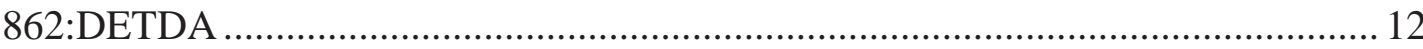

Figure 2.4 - Diagram of crosslinking reaction .................................................. 13

Figure 2.5 - Flowchart describing crosslinking algorithm ............................... 14

Figure 3.1 - Resulting structure after implementing the 'randomly rotate and translate' program for multiplying the initial 2:1 ratio structure ............................ 18

Figure 3.2 - a) Initial planar graphite structure and b) Graphite structure after equilibration

Figure 3.3 - Combining the three separate MD models to form the complete MD model of the composite interface

Figure 3.4 - Crosslink atom mass density along the z-axis for each crosslinked

structure

Figure 3.5 - Mass density profile along Z-axis of simulation box for graphite and polymer molecules

Figure 3.6 - Zoomed in interfacial mass density profile along Z-axis ..................... 24

Figure 3.7 - TEM image of carbon nanofiber embedded in an epoxy resin 25 
Figure 3.8 - EPON 862 and DETDA molecular mass densities along simulation box Z-axis for Non-crosslinked and $80 \%$ crosslinked structures 26

Figure 3.9 - Spatially averaged Von Mises stress for polymer and graphite atoms along Z-axis

Figure 3.10 - Spatially averaged per-atom potential energy along Z-axis. .28

Figure 4.1 - Molecular structures for single layer graphene sheet model highlighting the densification process.

Figure 4.2 - Equilibrated models for varying number of graphene layers before crosslinking.

Figure 4.3 - Crosslink dispersion as a function of z-axis coordinate for $80 \%$ crosslinked systems 35

Figure 4.4 - Simulated deformation characteristics for tension along the z-axis and shear in the $\mathrm{x}-\mathrm{z}$ direction

Figure 4.5 - Tensile and Shear stress vs strain curves for varying number of graphene layers in $80 \%$ crosslinked structures.

Figure 4.6 - Schematic representation for implementing MD results into MACGMC software to obtain data for GNP doped epoxy and GNP epoxy/Carbon Fiber composites 38

Figure 4.7 - MAC/GMC RUC for GNP doped epoxy / carbon fiber model 39

Figure 4.8 - Atom density profile along z-axis for a)1 layer b) 2 layers c) 3 layers and d) 4 layers of graphene

Figure 4.9 - Atomic stress before and after tensile deformation along the y-axis.....41 Figure 4.10 - MAC/GMC data for addition of GNPs with carrying number of layers and interfacial polymer crosslink density.

Figure 4.11 - MAC/GMC tensile modulus for GNP doped epoxy/carbon fiber models for varying GNP volume fractions and atomic thicknesses 44 
Figure 4.12 - Experimental and MAC/GMC tensile modulus for GNP doped epoxy/carbon fiber models.

Figure 4.13 - MAC/GMC transverse modulus for GNP doped epoxy/carbon fiber models for varying GNP volume fractions and atomic thicknesses ....................... 46

Figure 5.1 - Widely accepted models for the structure of graphene oxide............... 48

Figure 5.2 - The most stable confirmations of graphene oxide as found by DFT for a) epoxide oxygen only, b) hydroxyl molecules only, and c) a combination of epoxide and hydroxyl molecules

Figure 5.3 - Single and 4 layer graphene oxide structures containing $12 \%$ bonded oxygen species

Figure 5.4 - Fully equilibrated Single and 4 layer graphene oxide / epoxy structures containing $12 \%$ bonded oxygen species

Figure 5.5 - Polymer mass density profile viewed along the Z-Axis for single layer a) $12 \%$ bonded oxygen b) $24 \%$ bonded oxygen and 4 layer c) $12 \%$ bonded oxygen d) $24 \%$ bonded oxygen 54

Figure 5.6 - Visual representation of per-atom potential energy values (Kcal/molAngstrom) for single and 4 layer graphite oxide structures for varying oxygen species with $12 \%$ oxygen bonding 


\section{List of Tables}

Table 1.1 - Common carbon fibers and their corresponding mechanical properties.. 3

Table 4.1 - MAC/GMC Input Parameters for HexTow ${ }^{\circledR}$ AS4-GP/3K Fibers ...........39

Table 4.2 - Resulting data from MD simulations............................................... 41

Table 4.3 - Experimental results for epoxy/carbon fiber composites with varying

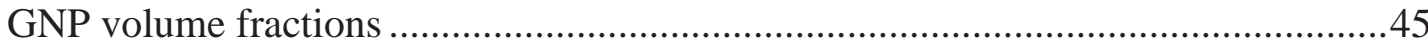

Table 5.1 - Mechanical properties derived from MD for oxidized graphene models along with those for $75 \%$ crosslinked pristine graphene MD models from chapter 4

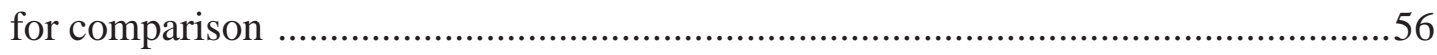

Table 5.2 - MAC/GMC results for 75\% corsslinked pristine and epoxide oxidized

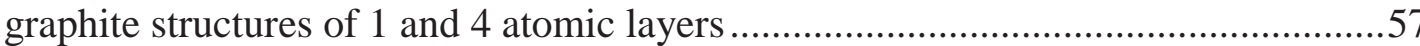




\section{PREFACE}

The work outlined in this dissertation was carried out in the department of Mechanical Engineering - Engineering Mechanics at Michigan Technological University over the period of August 2009 to December 2014. Portions of Chapters 2 and 3 have been presented in the following publication:

C.M. Hadden, B.D. Jensen, A. Bandyopadhyay, G.M.Odegard, A.Koo, R.Liang Composite Science and Technology 2013, 76, 92-99. (C) Elsevier Ltd

Portions of chapter 4 have been submitted for publication, and the information outlined in Chapter 5 could potentially be used for publication in the near future.

All of the writing in this dissertation was performed by yours truly, as well as all of the Molecular Dynamics research, which includes creating and deforming the models for extraction of material properties.

For help from other parties, I would first like to thank Dr. Gregory Odegard for his help with writing and formatting journal article submissions. I would like to thank Ananyo Bandyopadhyay for his molecular modelling contributions in Chapter 3, as well as Ben Jensen for help with programming. Also from Chapter 3, I would like to thank A. Koo and R. Liang for the use of their TEM image, which helped to reinforce my research. I would like to thank Dr. Julie King and Danielle Klimek for their contributions to Chapter 4, which include fabrication and mechanical testing of carbon composites for comparison to simulation results. Finally, I would like to thank Dr. Evan Pineda from NASA research for developing specific modelling scripts that would enable simulation data from Chapters 4 and 5 to be input into micromechanics software. 


\section{ACKNOWLEDGMENTS}

Obtaining my Ph.D. has been a great experience. I have been able to learn a lot about mechanical engineering, specifically mechanics of composite materials. I have also learned to do some pretty intricate programming and taken a few courses in materials science. I am very thankful for having such a great advisor in Dr. Gregory Odegard. He has helped to steer me through my doctorate and put me in a great position to obtain the job I wanted when accepting the challenge of obtaining my Ph.D.

I would also like to thank Dr. Reza Shahbazian-Yassar, Dr. Tolou Shokuhfar, Dr. Ranjit Pati, Dr. Gowtham Shankara, and Dr. Julia King for agreeing to be on my Ph.D. committee. The members of my committee changed from my initial proposal defense to my final defense, though all the aforementioned names have supported my research and provided valuable insight. During my Ph.D. I was able to travel and work with several research groups. From Dayton Air Force base I would like to thank Dr. Ajit Roy and Dr. Vikas Varshney. From NASA Langley I would like to thank Dr. Kris Wise. From NASA Glenn I would like to thank Dr. Evan Pineda and

Dr. Brett Bednarcyk. All of these gentleman have put in a lot of hard work in relation to Molecular Dynamics and micromechanics, without their guidance things would have been much more difficult for me.

This research would not have been possible without the funding and support from both NASA and the Air Force. Funding was provided by NASA under the Aircraft Aging and Durability Project (Grant NNX07AU58A) and the Revolutionary Technology Challenges Program (Grant NNX09AM50A); and by the Air Force Office of Scientific Research under the Low Density Materials Program (Grant FA9550-09-1-0375).

From Michigan Technologicaly University, I would like to thank the other members of my research group. Ananyo Bandyopadhyay helped me tremendously in getting a grasp on Molecular Dynamics and setting up my initial systems. Ben Jensen provided me with a lot of help with the various programs I had to write to create my models. I'd also like to thank Dr. Gowtham Shankara for all of his help with the cluster and his programming classes. All of those previously mentioned have been both great co-workers and great friends.

This has truly been an amazing experience for me. Houghton has not only provided me with a superior education, but has enabled me to enjoy the extra-curricular activities that make me who I am. I have been blessed to snowboard in the deepest powder, wakeboard on the flattest water, and surf some amazing waves on Lake Superior. I'd like to thank all of those outside of the university who were there to share in the excitement and for all of the high fives. I have been able to maintain the perfect balance of work and play, and I've had great people by my side for all of it. 
Finally, I would like to thank my family and friends that have been there from the beginning. My parents were the driving force behind me choosing Michigan Tech. They supported me throughout all the decisions I made while I was here, including that first summer spent away from home. My brother and sister have always encouraged me to do my best, and have been quick to tell others how proud they are of me. I will never forget that. Last but not least, I would like to say thank you to my lovely girlfriend, Jaime LeDuc, who has been there through all the ups and downs. She has been my most powerful inspiration for success.

Cameron Hadden

August 2014

Houghton, MI

USA 


\begin{abstract}
The thermoset epoxy resin EPON 862, coupled with the DETDA hardening agent, are utilized as the polymer matrix component in many graphite (carbon fiber) composites. Because it is difficult to experimentally characterize the interfacial region, computational molecular modeling is a necessary tool for understanding the influence of the interfacial molecular structure on bulk-level material properties. The purpose of this research is to investigate the many possible variables that may influence the interfacial structure and the effect they will have on the mechanical behavior of the bulk level composite. Molecular models are established for EPON 862-DETDA polymer in the presence of a graphite surface. Material characteristics such as polymer mass-density, residual stresses, and molecular potential energy are investigated near the polymer/fiber interface. Because the exact degree of crosslinking in these thermoset systems is not known, many different crosslink densities (degrees of curing) are investigated. It is determined that a region exists near the carbon fiber surface in which the polymer mass density is different than that of the bulk mass density. These surface effects extend $\sim 10 \AA$ into the polymer from the center of the outermost graphite layer. Early simulations predict polymer residual stress levels to be higher near the graphite surface. It is also seen that the molecular potential energy in polymer atoms decreases with increasing crosslink density.

New models are then established in order to investigate the interface between EPON 862-DETDA polymer and graphene nanoplatelets (GNPs) of various atomic thicknesses. Mechanical properties are extracted from the models using Molecular Dynamics techniques. These properties are then implemented into micromechanics software that utilizes the generalized method of cells to create representations of macro-scale composites. Micromechanics models are created representing GNP doped epoxy with varying number of graphene layers and interfacial polymer crosslink densities. The initial micromechanics results for the GNP doped epoxy are then taken to represent the matrix component and are re-run through the micromechanics software with the addition of a carbon fiber to simulate a GNP doped epoxy/carbon fiber composite. Micromechanics results agree well with experimental data, and indicate GNPs of 1 to 2 atomic layers to be highly favorable.

The effect of oxygen bonded to the surface of the GNPs is lastly investigated. Molecular Models are created for systems with varying graphene atomic thickness, along with different amounts of oxygen species attached to them. Models are created for graphene containing hydroxyl groups only, epoxide groups only, and a combination of epoxide and hydroxyl groups. Results show models of oxidized graphene to decrease in both tensile and shear modulus. Attaching only epoxide groups gives the best results for mechanical properties, though pristine graphene is still favored
\end{abstract}





\section{CHAPTER 1: INTRODUCTION}

\subsection{Material Overview}

Thermoset epoxy/carbon fiber composites have long been one of the primary material choices for modern aerospace applications. These materials exhibit an excellent strength to weight ratio and can be easily manufactured to have specific performance characteristics. The overall material properties of epoxy composites depend not only on the individual properties of both the carbon fiber and epoxy matrix, but also the physical and chemical conditions of the fiber/matrix interface. ${ }^{1} \mathrm{~A}$ good bond between the fiber and matrix enables improved load transfer from the matrix to the fibers which results in greater composite stiffness and strength, but lower toughness because of the absence of crack deflection mechanisms at the interface. In contrast, a weak bond between fiber and matrix will provide lower composite strength with a higher toughness. ${ }^{2}$ The molecular behavior of the epoxy matrix and carbon fiber surface can provide valuable insight to the interface, and thereby the mechanical behavior of the entire composite.

\section{Epoxy Resins}

Thermoset epoxy resins derive their name from the 3-membered epoxide ring found in their molecular structure, as shown in Figure 1.1(a). The epoxide ring is a high energy molecular structure and this energy is reduced by reacting to form bonds with different molecules, commonly known as hardeners. Initially both the epoxy and the hardener components are a viscous liquid, and when mixed they polymerize and harden. This process is often referred to as curing. It is important to note that the extent of curing in thermoset epoxies is unknown, and will be an important variable for the research performed herein. Different epoxy resins can have different amounts of epoxide groups per molecule, and different epoxy molecules will produce a polymer with unique mechanical properties. Hardeners often contain a reactive 
hydrogen group such as an amine, shown in Figure 1.1(b), or acid. Different hardeners will also produce a polymer with unique properties. ${ }^{3}$
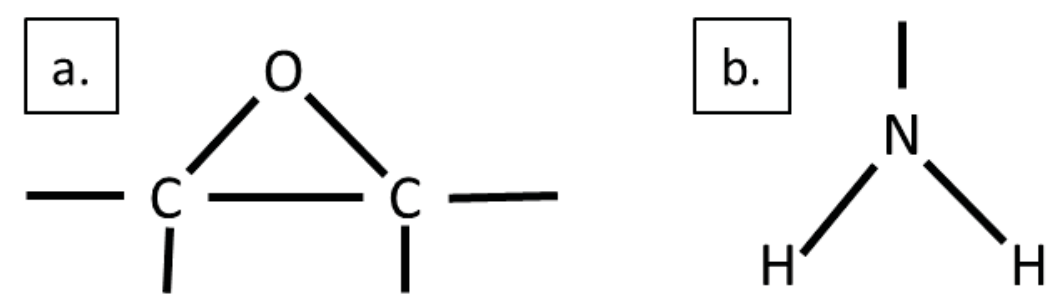

Figure 1.1 a) Epoxide ring containing two reactive carbon atoms and one oxygen atom. b) Amine group containing two reactive hydrogen atoms and one nitrogen atom.

Fully cured epoxy resins have many desirable properties such as: excellent adhesion to many materials, good mechanical and electrical properties, high heat resistance, and resistance to chemicals and moisture. ${ }^{3}$ Because of this they have many applications, from aerospace composites to house-hold adhesives.

\section{Carbon Reinforcements}

The most common form of carbon reinforcement used in modern composites are carbon fibers. These fibers are usually manufactured by the oxidation/carbonization of polyacrylonitrile (PAN) fibers. ${ }^{1}$ Carbon fibers can be manufactured to exhibit an array of material properties, and are commonly categorized as either high modulus (Type I) or high strength fibers (Type II). ${ }^{4}$ High modulus fibers require heat treatment during manufacturing of $>2000^{\circ} \mathrm{C}$, while high strength fibers are manufactured at $\sim 1500{ }^{\circ} \mathrm{C} .{ }^{4}$ The mechanical properties for carbon fibers depend strongly on the manufacturing process and the test method used to determine the desired property. A list of common carbon fibers and there corresponding mechanical properties is provided in Table 1.1. Carbon fibers are used in composites by weaving individual fibers into a desirable pattern and combining them with a polymer matrix. Figure 1.2 depicts a highly accepted structure of a carbon fiber. It can be seen that the surface molecules are much more organized that that of the core, and the molecules on the surface are overlapping sheets composed of aromatic carbon rings. 
Table 1.1 Common carbon fibers and their corresponding mechanical properties ${ }^{4}$

\begin{tabular}{|c|c|c|c|c|}
\hline Fiber Type & Modulus (Gpa) & Tensile Strength (Gpa) & Tensile Elongation (\%) & Examples \\
\hline Ultra High Modulus & 524 & 2.1 & 0.4 & P75(a) \\
\hline High Modulus (Type I) & $350-360$ & 2.5 & 0.7 & $\begin{array}{l}\text { HMS-4(b) } \\
\text { G50 (c) }\end{array}$ \\
\hline Intermediate Modulus & $280-310$ & $4.8-5.5$ & $1.8-1.95$ & $\begin{array}{c}\text { IM-6 (b) } \\
\text { IM-7 (b) } \\
\text { HiTex } 42(d) \\
\text { Toray } 40 \text { (e) }\end{array}$ \\
\hline High Elongation & $240-260$ & $4.5-4.8$ & $1.9-2.0$ & $\begin{array}{l}\text { AS-6 (b) } \\
\text { Toray } 700 \\
\text { (e) }\end{array}$ \\
\hline $\begin{array}{l}\text { Intermediate Strength } \\
\text { (Type II) }\end{array}$ & 235 & 4.1 & $1.5-1.7$ & $\begin{array}{l}\text { AS-4 (b) } \\
\text { T300 (a) }\end{array}$ \\
\hline
\end{tabular}

(a) Amoco Chemical Corp.

(b) Hercules Inc.

(c) BASF Fibers

(d) Owens Corning Fiberglass Corp.

(e) Toray Industries Inc., Osaka Japan
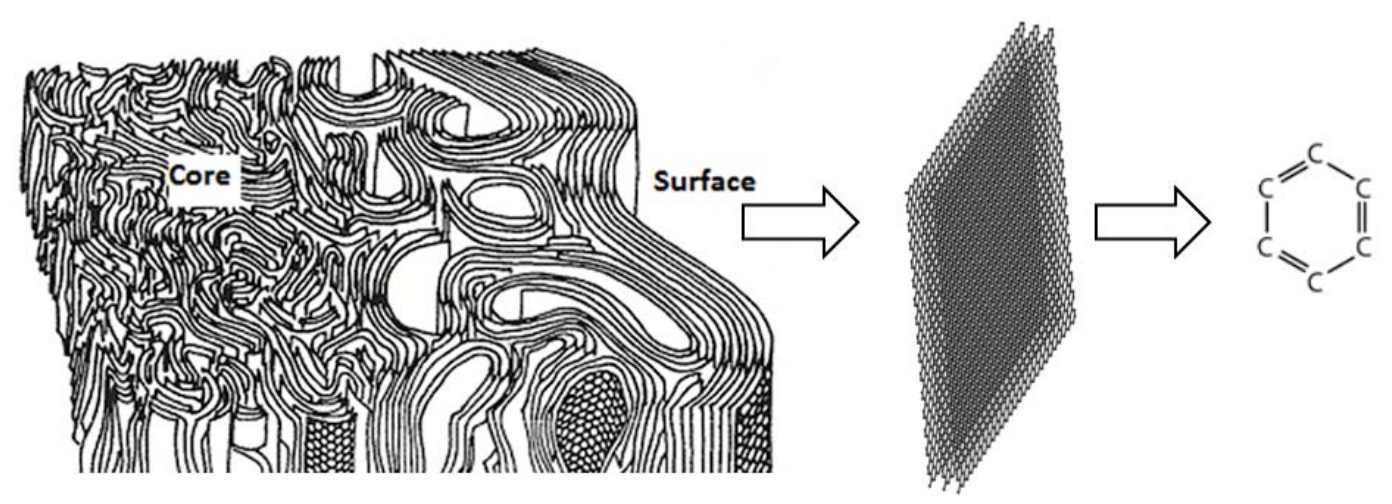

Figure 1.2 Predicted internal structure for carbon fibers, surface atom structures, and aromatic carbon ring structure. Image courtesy of NASA Report 4084 @ L.T. Drzal

Surface treatments are often applied to carbon fibers to improve bonding to matrix polymers. ${ }^{5}$ Chemical treatments can increase the surface energy of carbon fibers and promote bonding to matrix polymers by adding reactive species that are 
able to bond to the certain groups within the matrix polymer molecules. Fibers can also be coated with 'sizings' to protect them during the fabrication process, and in some cases increase bonding. ${ }^{11}$ Carbon nanostructures such as nanotubes ${ }^{6,7}$ and graphene nanoplatelets (GNPs) $)^{8,9}$ can also be used within a polymer matrix to create nano-composites. They have also been shown to increase mechanical properties when added to carbon fiber/epoxy composites. ${ }^{7,10}$

\subsection{Molecular Dynamics}

Molecular Dynamics (MD) can be defined as a form of computer simulation in which atoms and molecules interact for a defined period of time by approximations of known physics, thus describing the motion of the atoms. MD is

based on statistical mechanics, and requires a lot of computational power. There are four types of MD simulations most commonly used: ${ }^{12}$

1. Canonical ensemble (NVT ensemble)- The NVT ensemble does not allow the system to undergo changes in number of moles $(\mathrm{N})$, volume $(\mathrm{V})$, or temperature (T). A thermostat is needed to help steer the exchange of exothermic and endothermic processes within the system. Different thermostats are available to remove energy from the system in a manner 'realistic' for specific applications.

2. Isothermal-Isobaric (NPT ensemble)- The NPT ensemble does not allow for change to number of moles $(\mathrm{N})$, pressure $(\mathrm{P})$, or temperature $(\mathrm{T})$. A thermostat and barostat are necessary to help control temperature and pressure, respectively. The volume is allowed to fluctuate based on the temperature and pressure of the system. 
3. Microcanonical ensemble (NVE ensemble)- The NVE ensemble does not allow the system to undergo changes in number of moles $(\mathrm{N})$, volume $(\mathrm{V})$, or energy (E). By keeping the energy term constant, it creates an adiabatic process and does not allow for heat exchange. Overall energy is kept constant and is continuously changing between kinetic and potential energies.

4. Isoenthalpic-Isobaric (NPH ensemble)- The NPH ensemble does not allow change to number of moles $(\mathrm{N})$, pressure $(\mathrm{P})$, or enthalpy $(\mathrm{H})$. This ensemble denotes volume $(\mathrm{V})$ as a dynamic variable relating to the coordinates of all the atoms in the system. Kinetic energy is defined as PV. Enthalpy can then be defined as $\mathrm{H}=\mathrm{E}+\mathrm{PV}$, where $\mathrm{H}$ is kept constant and internal energy (E) and kinetic energy PV are allowed to change in response to one another.

Energy minimization, or molecular minimization (MM), simulations are used in conjunction with MD to reduce the overall energy of a system. MM simulations make small adjustments to molecular geometries searching for low energy confirmations. The ultimate goal in equilibrating an MD model is to search for the lowest possible energy confirmation, as that is what occurs in nature based on the laws of thermodynamics.

The use of computational methods for studying material behavior has really taken off in the last decade. MD gives researchers insight to the molecular behavior of a material, something that experimental techniques have yet to explain. The most disputed aspect of MD is the timescale for which the simulations are conducted. Most MD simulations occur over a few hundred picoseconds, while longer simulations may span a few nanoseconds. Because of this, they are difficult for comparison with experimental data. As MD modeling techniques continue to improve, they will enable researchers to develop new materials outside of the lab setting and will provide information that will steer future experimental research. By teaming up with experimental researchers, $\mathrm{MD}$ can help lessen time in developing new materials and the money associated with experimental techniques. 
Much research has been done using MD for thermoset epoxies. Early MD methods for studying thermoset epoxies utilized bead-spring models ${ }^{13,14}$ and MonteCarlo simulations based on bond fluctuation models ${ }^{15-17}$. More recent techniques for studying epoxies utilize detailed molecular structures and produce models of much larger size (more atoms). Recent models have also focused heavily on the crosslinking mechanisms that occur during curing. ${ }^{18-21}$ These studies have lead the way to developing models for many epoxy/carbon composite systems. There has been extensive research performed using MD models to investigate thermoset polymers containing nanotubes ${ }^{22-28}$, nanoparticles ${ }^{29-31}$, and in the presence of a graphite surface (representing either graphene nanoparticles or the surface of a carbon fiber). ${ }^{33-37}$ The interface between epoxy and carbon reinforcement is investigated in nearly all of the aforementioned references. Mechanical properties have been derived from MD models for a variety of epoxy/carbon systems. ${ }^{36-40}$ Although these studies have given valuable information regarding the physical nature of the interfacial region, including an estimate of material properties on the molecular level, there has been little effort to implement this molecular data into a larger scale model that will enable comparison to experimental results for similar systems.

The objective of this research was to first establish an efficient and accurate procedure for developing large scale models of EPON 862-DETDA epoxy in the presence of a carbon fiber surface. The interfacial characteristics of the polymer were analyzed for multiple crosslink densities (degrees of curing). The initial models were validated with experimental TEM images for similar systems. The methodology used to establish the initial model would then be used to create new models of EPON 862DETDA epoxy with embedded graphene nanoplatelets (GNPs). Mechanical properties were extracted using MD techniques and the effects of GNP atomic thickness and polymer crosslink density were investigated. The mechanical 
properties derived from the MD models were then implemented into a micromechanics model based on the generalized method of cells. This micromechanics model would enable comparison to experimental results for both a GNP doped epoxy specimen, as well as a GNP doped epoxy / carbon fiber composite. Lastly, the effect of adding oxygen functional groups to the surface of graphene nanoplatelets was investigated. MD models were created with GNPs of multiple atomic thicknesses with a variety of oxygen species attached in multiple concentrations. For this final study, polymer crosslink density was kept at a constant. Again, micromechanics models were used for comparison to experimental results.

\section{CHAPTER 2: MOLECULAR MODELING PROCEDURES ${ }^{1}$}

This section describes the procedures used for establishing molecular models. The Force Field chosen for this research is highlighted, followed by the procedure for building the initial model of a 2:1 stoichiometric ratio of EPON 862:DETDA that would be used as the building block for all larger models. The crosslinking procedure used throughout this research will lastly be discussed.

\subsection{Force Field and Simulation Parameters}

The force field used in this research was the OPLS United Atom force field developed by Jorgensen and co-workers. ${ }^{41-44}$ This force field calculates the total energy of the system as the sum of all the individual energies associated bond, angle, dihedral and 12-6 Lennard-Jones interactions. A visual representation for each energy contribution can be seen in Figure

${ }^{1}$ Portions of text in this chapter have been reprinted with permission from C.M. Hadden, B.D. Jensen, A. Bandyopadhyay, G.M.Odegard, A.Koo, R.Liang Composite Science and Technology 2013, 76, 9299. (C) Elsevier Ltd. Please refer Appendix B for copyright information. 

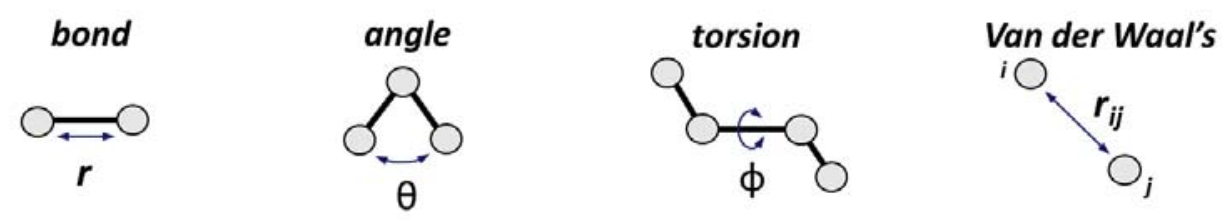

Figure 2.1 A visual representation of each energy contribution that is considered by the OPLS United Atom force field.

The equations for each energy contribution are given as follows. The bond energy contribution is a simple quadratic equation given as:

$$
E_{\text {bonds }}=\sum_{\text {bonds }} K_{r}\left(r-r_{0}\right)^{2}
$$

$K_{r}$ is a force constant, $r$ is the distance between the two bonded atoms, and $r_{0}$ is the equilibrium bond distance for the two bonded atoms. The energy associated with bond-angle bending is also a quadratic equation and is given as:

$$
E_{\text {angles }}=\sum_{\text {angles }} K_{\theta}\left(\theta-\theta_{0}\right)^{2}
$$

$K_{\theta}$ is a force constant, $\theta$ is the angle between the bonds, and $\theta_{0}$ is the equilibrium bond angle. The dihedral energy is calculated as:

$$
\begin{gathered}
E_{\text {dihedrals }}=\frac{V_{1}}{2}[1+\cos \emptyset]+\frac{V_{2}}{2}[1-\cos (2 \varnothing)]+\frac{V_{3}}{2}[1+\cos 3 \emptyset]+\frac{V_{4}}{2}[1- \\
\cos 4 \varnothing]
\end{gathered}
$$

where $V_{1}, V_{2}, V_{3}$ and $V_{4}$ are coefficients of a Fourier series ${ }^{42,44}, \Phi$ is the dihedral angle. The van der Waal's interactions are represented by the 12-6 Lennard Jones equation:

$$
E_{i j}=4 \varepsilon_{i j}\left[\left(\frac{\sigma_{i j}}{r_{i j}}\right)^{12}-\left(\frac{\sigma_{i j}}{r_{i j}}\right)^{6}\right]
$$

Where $\sigma$ is the equilibrium spacing parameter, and was considered to be the arithmetic mean of the individual parameters of atoms $i$ and $j . \varepsilon$ is the well depth parameter and was considered to be the geometric mean of the values given for atoms $i$ and $j$. Here, $r$ denotes the distance between the non-bonded atoms $i$ and $j$. 
Charges were not considered in this work, as they require a lot of computational power to calculate. Also graphite structures are known to be neutral of charge and will be the main influence on interfacial atomic structure, thereby deeming charges unnecessary for this particular research.

\subsection{Modeling Initial Structures}

This section describes the procedure for establishing the 2:1 stiochiometric model of EPON 862 monomer: DETDA hardener. The procedure for multiplying this initial small structure into a much larger system is different depending on the desired simulation data, and hence will be described in the appropriate chapters. The initial 2:1 ratio was produced in a similar matter for all models, utilizing the LAMMPS (Large Scale Atomic/Molecular Massively Parallel Simulator) ${ }^{12,45}$ software package for all of the MM and MD simulations described in the following.

The initial uncrosslinked polymer molecular structure was established using a procedure similar to that of Bandyopadhyay et al. ${ }^{20}$, consisting of the EPON 862 (Diglycidyl ether of Bisphenol-F) monomer and the DETDA (Diethylene Toluene Diamine) hardener shown in Figure 2.2. A stoichiometric ratio of 2 molecules of EPON 862 and 1 molecule of DETDA was placed in a 10 x 10 x $10 \AA$ MD simulation box with periodic boundary conditions. The 2:1 stoichiometric ratio was chosen due to the fact that if all possible crosslinks were formed, the polymer would achieve $100 \%$ crosslink density (full curing) allowing the polymer to exhibit the best possible material properties. The initial atomic coordinates file was written in the native LAMMPS format with the OPLS United Atom force field, as previously described. The non-bonded van der Waal's interactions were modeled with in interaction cutoff radius of $10 \AA$. The use of the OPLS United Atom force field allows for modeling of $\mathrm{CH}_{3}, \mathrm{CH}_{2}, \mathrm{CH}$, and alkyl groups as single united atoms with their corresponding masses. The described polymer model utilizes united atoms 
structures for all applicable groups, except for the $\mathrm{C}$ and $\mathrm{H}$ atoms in the phenyl rings for both monomer and hardener molecules along with one $\mathrm{CH}_{3}$ group directly connected to the phenyl ring of the DETDA molecule. Thus, the use of united atoms reduced the 2:1 structure from 117 atoms to 83 atoms. The location of each united atom is show in Figure 2.2, with 31 total atoms in the molecule of EPON 862 and 21 in the molecule of DETDA.
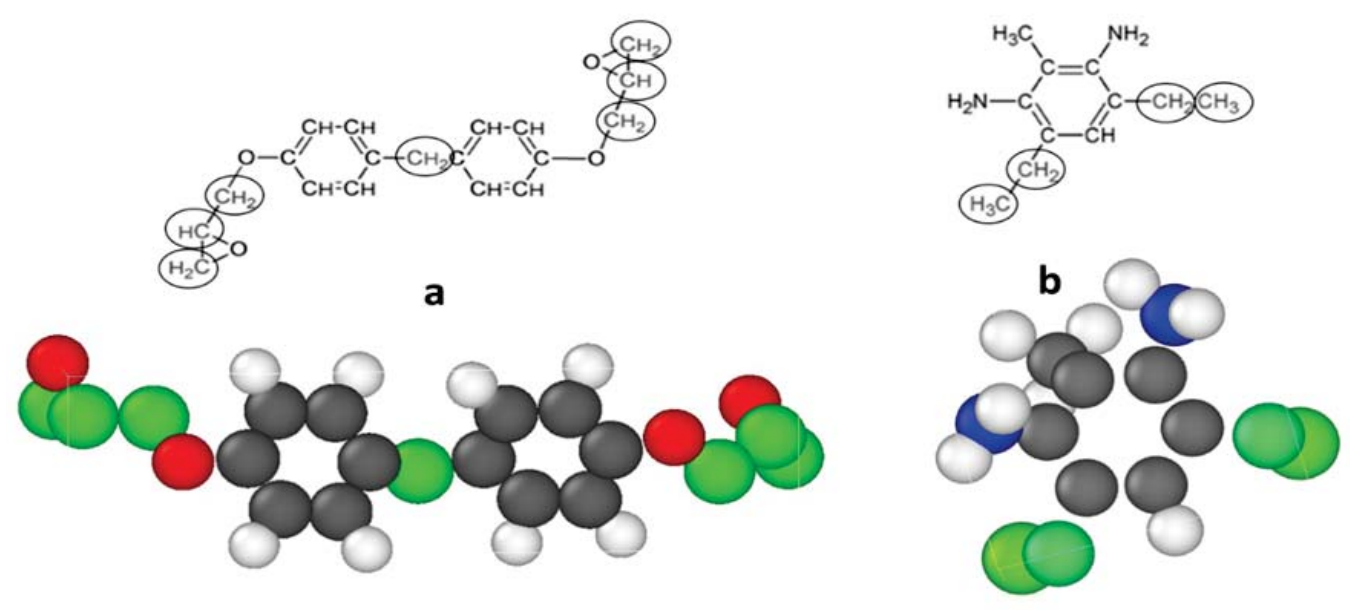

Figure 2.2 Molecular structures of a) EPON 862 and b) DETDA shown with simulated image. Top chemical structures show united atoms to be circled, corresponding to the green beads in the simulated image.

Creating a coordinates file requires defining specific atom types, which were easily located in the OPLS United Atom parameters manual. Bonds were defined according to the atom types for each bonded atom, again these were easily obtained. All OPLS United Atom force field parameters used in this work are given in Appendix A.5. Angle and Dihedral parameters are minimally defined in literature, and therefor required several assumptions to be made.

\section{Assumptions for angle types:}

1. $\mathrm{C}-\mathrm{C}-\mathrm{N}$ was given the same parameters as $\mathrm{C}-\mathrm{C}-\mathrm{C}$

2. $\mathrm{CH}_{2}{ }^{-} \mathrm{CH}-\mathrm{CH}_{2}, \mathrm{CH}_{3}{ }^{-} \mathrm{CH}-\mathrm{CH}_{2}, \mathrm{CH}_{3}{ }^{-} \mathrm{CH}-\mathrm{CH}_{3}, \mathrm{CH}^{-} \mathrm{CH}-\mathrm{CH}_{3}$, and $\mathrm{CH}^{-} \mathrm{CH}-\mathrm{CH}$ all utilized the same parameters 
3. $\mathrm{CH}_{3}{ }^{-} \mathrm{CH}_{2}-\mathrm{C}$ was given the same parameters as $\mathrm{CH}-\mathrm{CH}_{2}-\mathrm{CH}$

4. $\mathrm{CH}-\mathrm{CH}_{2}-\mathrm{O}$ was given the same parameters as $\mathrm{CH}_{2}-\mathrm{CH}-\mathrm{O}$

5. $\mathrm{CH}_{2}-\mathrm{O}-\mathrm{CH}, \mathrm{C}-\mathrm{O}-\mathrm{CH}_{2}, \mathrm{CH}_{2}-\mathrm{O}-\mathrm{CH}_{3}, \mathrm{CH}_{2}-\mathrm{O}-\mathrm{CH}_{2}$, and $\mathrm{CH}-\mathrm{O}-\mathrm{CH}$ all utilized the same parameter set

\section{Assumptions for dihedral types:}

1. $\mathrm{C}_{\mathrm{a}}-\mathrm{C}_{\mathrm{a}}-\mathrm{CH}_{2}-\mathrm{C}_{\mathrm{a}}$ and $\mathrm{CH}_{3}-\mathrm{CH}_{2}-\mathrm{C}_{\mathrm{a}}-\mathrm{C}_{\mathrm{a}}$ were given same parameters as $\mathrm{C}_{\mathrm{a}^{-}}-\mathrm{C}_{\mathrm{a}^{-}}$ $\mathrm{C}_{\mathrm{a}^{-}} \mathrm{C}_{\mathrm{a}}$, where $\mathrm{C}_{\mathrm{a}}=$ aromatic carbon

2. All dihedral $\mathrm{N}$ atoms in DETDA were considered as $\mathrm{C}$ atoms

3. C-O-CH $2-\mathrm{CH}, \mathrm{CH}-\mathrm{O}-\mathrm{CH}-\mathrm{CH}$, and $\mathrm{CH}_{2}-\mathrm{O}-\mathrm{CH}-\mathrm{CH}_{2}$ were given same parameters

4. $\mathrm{CH}_{2}-\mathrm{CH}-\mathrm{CH}_{2}-\mathrm{O}, \mathrm{C}-\mathrm{CH}-\mathrm{CH}_{2}-\mathrm{O}$ and $\mathrm{CH}_{2}-\mathrm{CH}-\mathrm{CH}_{2}-\mathrm{O}$ were given same parameters

The main contribution to the potential energy of the system is caused by bond energy, as more energy is required to alter bond distances than to distort an angle or dihedral angle from equilibrium. In saying this, the assumptions made should not significantly affect the overall potential energy of the model.

After establishing an atomic coordinates file, the initial 2:1 structure was subjected to four MM minimizations and three MD simulations in order to minimize internal forces and reduce internal residual stresses created by the initial construction of bonds, bond angles, and bond dihedrals. The MM simulations were run with a conjugate gradient stopping criterion which utilizes stored information from successive line searches to steer the atomic configuration to a minimal energy confirmation. ${ }^{12}$ At each iteration the force gradient is combined with information from the previous iteration to compute a new search direction perpendicular (conjugate) to the previous search direction. The minimization ceases when no moves can be made to further reduce the energy of the system. The MD simulations utilize the NVT ensemble with each simulation lasting $100 \mathrm{ps}$ at $300 \mathrm{~K}$. A timestep of 0.2 femtoseconds was utilized for initial equilibration steps and all other MD simulations described in this work. A Nose/Hoover thermostat and barostat was 
implemented for temperature and pressure control, respectively. ${ }^{46}$ After equilibrating the initial 2:1 structure, it was ready to be inserted into a program that would randomly rotate and translate it to fill a desired volume. The equilibrated 2:1 structure can be seen in Figure 2.3

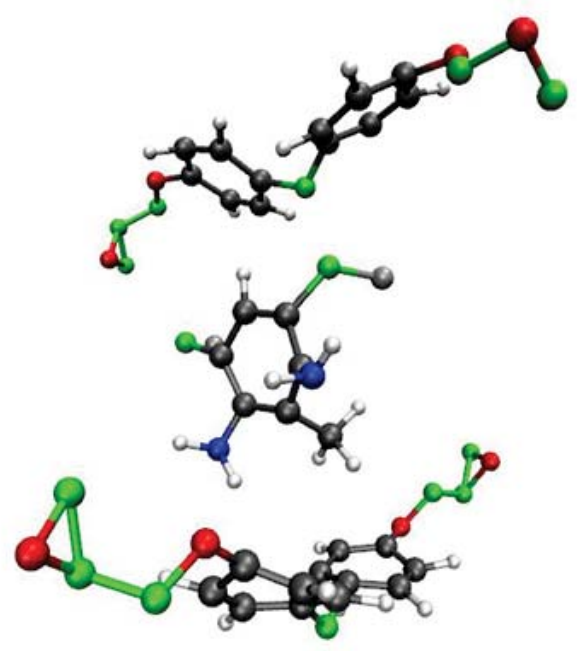

Figure 2.3 A visual representation of the fully equilibrated 2:1 ratio of EPON 862:DETDA.

\subsection{Crosslinking Procedure}

The equilibrated model was crosslinked based on the root mean square (RMS) distance between the $\mathrm{CH} 2$ groups of the EPON 862 and the $\mathrm{N}$ atoms of DETDA molecules, similar to the method used by Yarovsky and Evans ${ }^{47}$ and Bandyopadhyay et al. ${ }^{20}$ The modeled crosslinking reaction process is shown in Figure 2.4. Simultaneous breaking of the $\mathrm{CH} 2-\mathrm{O}$ bonds in the epoxide ends of the EPON 862 molecules and N-H bonds of the DETDA molecules enable the activated $\mathrm{CH} 2$ ends available to form crosslinks with activated $\mathrm{N}$ atoms of the DETDA molecules. A particular activated $\mathrm{N}$ can form a crosslink with the activated $\mathrm{CH} 2$ for any adjacent EPON 862 molecule within a specified cut-off distance. Three assumptions were made for the crosslinking process: 
1) Both primary amines in DETDA were assumed to have the same reactivity

2) The $\mathrm{CH} 2-\mathrm{O}$ and $\mathrm{N}-\mathrm{H}$ bonds were broken simultaneously (Figure $2.4 \mathrm{~A}$ )

3) The newly formed $\mathrm{CH} 2-\mathrm{N}$ and $\mathrm{O}-\mathrm{H}$ bonds were created simultaneously (Figure 2.4 B)
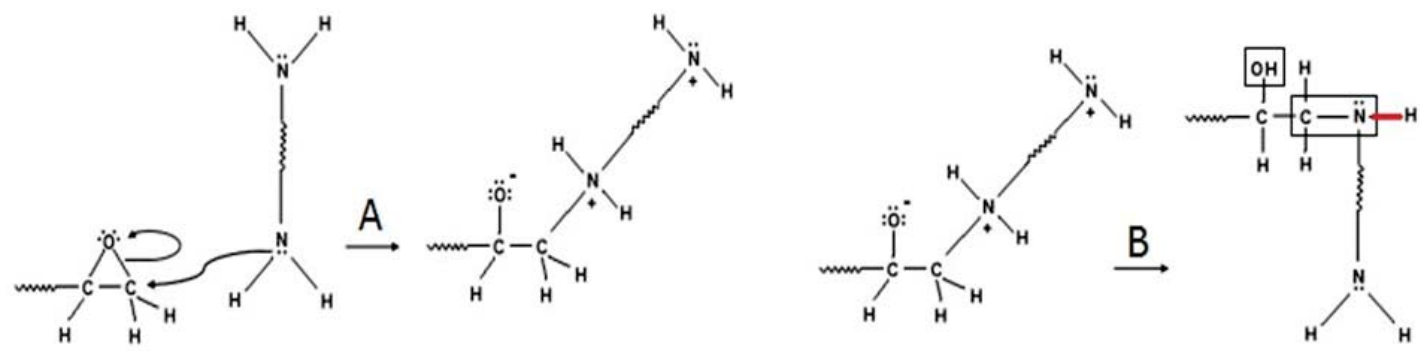

Figure 2.4. Crosslinking reaction: (A) Formation of primary C-N bond, leaving a negative charge on the oxygen and a positive charge on the nitrogen. (B) The negatively charged oxygen abstracts a proton from the neighboring amine, resulting in an alcohol group and an amine group. The reacted nitrogen is capable of forming another crosslink by the same reaction, breaking the $\mathrm{N}-\mathrm{H}$ bond shown in red.

A flow chart describing the modeled crosslink reaction procedure is shown in Figure 2.5. After selecting an RMS cut-off distance, which affects the ultimate crosslink density of the MD model and overall energy of the model (larger bond distances produce higher energy), the RMS distances were calculated between each pair of $\mathrm{N}$ atoms and $\mathrm{CH} 2$ united atoms selecting atoms within a specified cut-off distance. If multiple pairs (for a particular $\mathrm{N}$ or $\mathrm{CH} 2$ ) were found within the cut-off, the closest pair was chosen for the crosslink reaction. Pairs outside the cut-off were not considered. The covalent bonds corresponding to these crosslink reactions were inserted into the MD model. The next step of the process was to identify and form the appropriate secondary bonds between the $\mathrm{H}$ atoms from the broken $\mathrm{NH} 2$ groups and the $\mathrm{O}$ - atoms of the broken epoxide ends. This bond was formed based on the closest RMS distances between the $\mathrm{O}$ - and $\mathrm{H}+$ atoms from the previous step. 
Because the newly formed bonds were capable of forming at distances greater than that of equilibrium, a multistep bond relaxation process was implemented (as shown in Figure 2.5) to avoid excessively high energies within the system. Newly formed bonds were run through a minimization and quick NVT simulation with a relaxed bond force constant for cut-off distances $>5 \AA$.

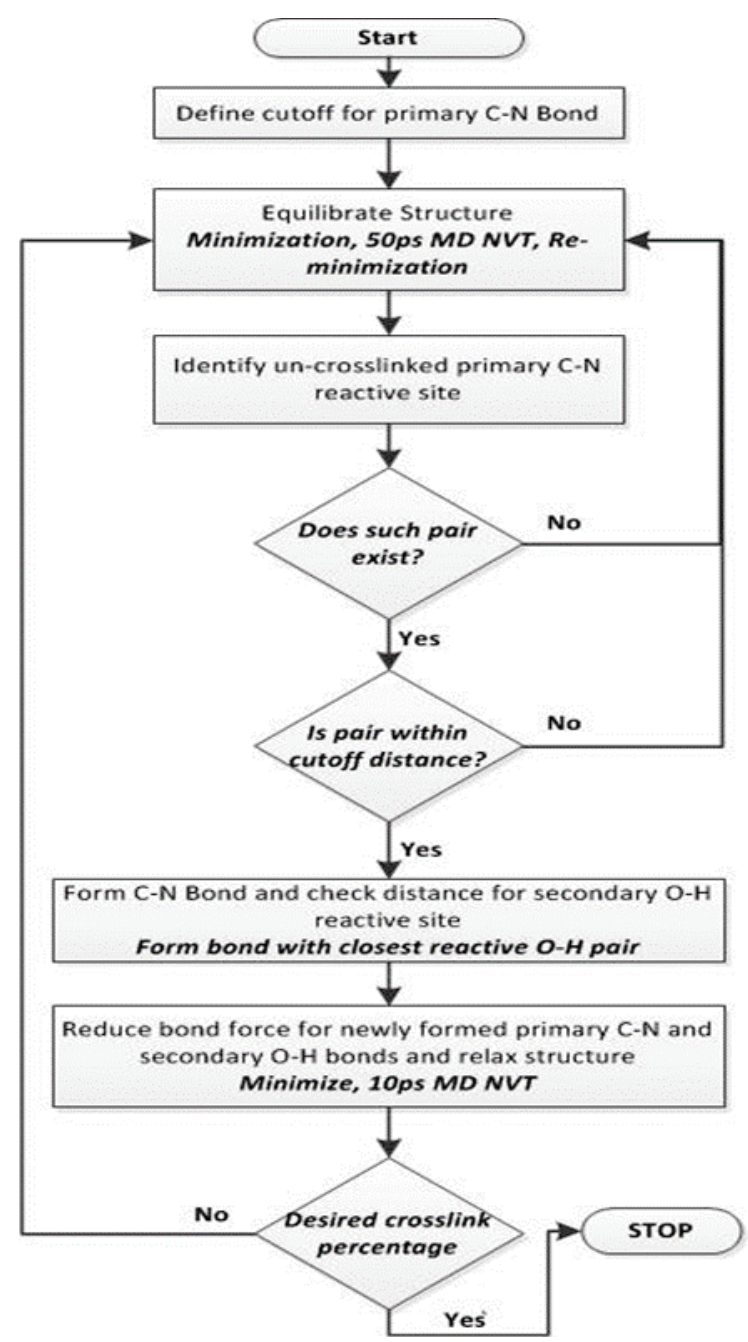

Figure 2.5. Flowchart describing crosslinking algorithm.

Initial runs of this crosslinking method enabled for high amounts of crosslinks to be formed for low cut-off distances. The term crosslink density is used to describe the ratio of the total number of crosslinks that were formed in relation to the maximum number of crosslinks that could be formed. For example, an epoxy network having 10 out of 20 crosslinks would have a crosslink density of $50 \%$. For 
all models described in this work, an initial cut-off of $3.5 \AA$ was incorporated which achieved an average of $\sim 6 \%$ crosslink density in each model. For each iteration thereafter, the cut-off distance was slightly increased in order to achieve increases in crosslink density around that original value of $\sim 6 \%$ per run. In order to maintain a realistic crosslinking mechanism, cutoffs did not exceed $6 \AA$. By not increasing crosslink density by large amounts per run, the system was able to move more freely from iteration to iteration. It was discovered early on that by crosslinking large amounts of polymer (>10\% crosslink density per run), the molecules within the system would become very restricted and successive crosslinking simulations could not achieve nearly as high of crosslink density. Also, by not having cut-off distances greater than $6 \AA$, it significantly reduced the chances of creating a bond through an aromatic ring. This is known as 'ring spearing', and theoretically will not occur in nature. It is also important to note that as crosslink density increased (>50\% crosslink density), the duration of the NVT simulation for equilibration would increase. Initially systems were equilibrated for 50ps between runs. For later crosslink iterations, the NVT equilibration time was increased up to 300ps in order to better 'stir' up the molecules and achieve higher crosslink densities per run. By using this method, the highest crosslink density achieved was $85 \%$ which required $20+$ runs of the crosslinking program and required $\sim 5 \mathrm{~ns}$ of simulation time. Higher crosslink densities could have been achieved by further increasing the cut-off distance, though larger cut-off distances become less representative of a 'realistic' crosslinking mechanism.

Many different crosslink densities were investigated in this work, as the exact extent to which a thermoset epoxy crosslinks is unknown. Because of this, it is important to look at the influence of crosslink density on both molecular behavior and mechanical properties. Crosslink densities used in each model will be described in more detail in upcoming chapters. 


\section{CHAPTER 3: MODELING THE FIBER / MATRIX INTERFACE ${ }^{1}$}

This chapter describes the first models that were created in order to investigate the interaction between EPON 862-DETDA polymer atoms and a carbon fiber surface. The effects of crosslink density were taken into consideration. This initial study utilized a unique method for equilibrating the models to the appropriate density. All modeling details are highlighted in the following.

\subsection{Introduction}

The interface between a polymer matrix and carbon reinforcement has long been a topic of interest. It was recognized early on that a region exists near the reinforcement surface in which polymer atoms behave differently than bulk. ${ }^{2-4}$ In relation to MD, Stevens ${ }^{33}$ used a course-grained model to simulate interfacial fracture between a highly crosslinked polymer network and a solid surface. Mansfield and Theodorou ${ }^{32}$ investigated the interface between graphite and a glassy polymer and determined that a $10 \AA$ thick interfacial region existed in the polymer that was structurally different from that of the bulk polymer. Recently, Chunyu Li et al. ${ }^{37}$ used MD simulations to observe the interface of a crosslinked thermoset polymer in the presence of a graphite surface. Although these studies have provided valuable information regarding the physical nature of the interfacial region in composites materials, they have not addressed the influence of crosslink density on the molecular structure of graphite/epoxy composite interfaces.

The purpose of this study was to determine the effect of crosslink density on the molecular structure of the fiber/matrix in a graphite/epoxy (EPON 862/DETDA) composite material. MD models were constructed for a wide range of crosslink

${ }^{1}$ Portions of text in this chapter have been reprinted with permission from C.M. Hadden, B.D. Jensen, A. Bandyopadhyay, G.M.Odegard, A.Koo, R.Liang Composite Science and Technology 2013, 76, 9299. (C) Elsevier Ltd. Please refer Appendix B for copyright information. 
densities and the molecular structure of the interface corresponding to each crosslink density was determined. The simulations predicted a polymer molecular structure at the interface that is different than that of the bulk polymer and is dependent on the crosslink density.

\subsection{Modeling Procedures}

The initial un-crosslinked polymer model began as the 2:1 stoichiometric ratio of the EPON 862 monomer and the DETDA hardener outlined in section 2.2 (page 9). The initial 2:1 structure was then implemented into a program that randomly rotated the molecular coordinates, and then translated these randomized coordinates along the $\mathrm{x}, \mathrm{y}$, and $\mathrm{z}$ axes to fill a specified volume. The resulting structure consisted of many randomly oriented clusters of the small 2:1 ratio stacked loosely together in a manner much like that of a simple cubic crystal structure, as seen in figure 3.1. The 2:1 coordinates file was multiplied into a low density structure consisting of 15,936 polymer atoms with a ratio of EPON 862:DETDA of 394:192 and a simulation box size of 64 × 66 × $115 \AA$, equating to a density of $\sim 0.5$ $\mathrm{g} / \mathrm{cc}$. The larger polymer model was then allowed to equilibrate into a uniform lowdensity liquid using a slow relaxation procedure performed over a cycle of $6 \mathrm{MM}$ and 3 MD simulations. All MD simulations were conducted with the NVT ensemble for $100 \mathrm{ps}$ at $300 \mathrm{~K}$. This equilibration was done before the addition of a simulated graphite surface. 


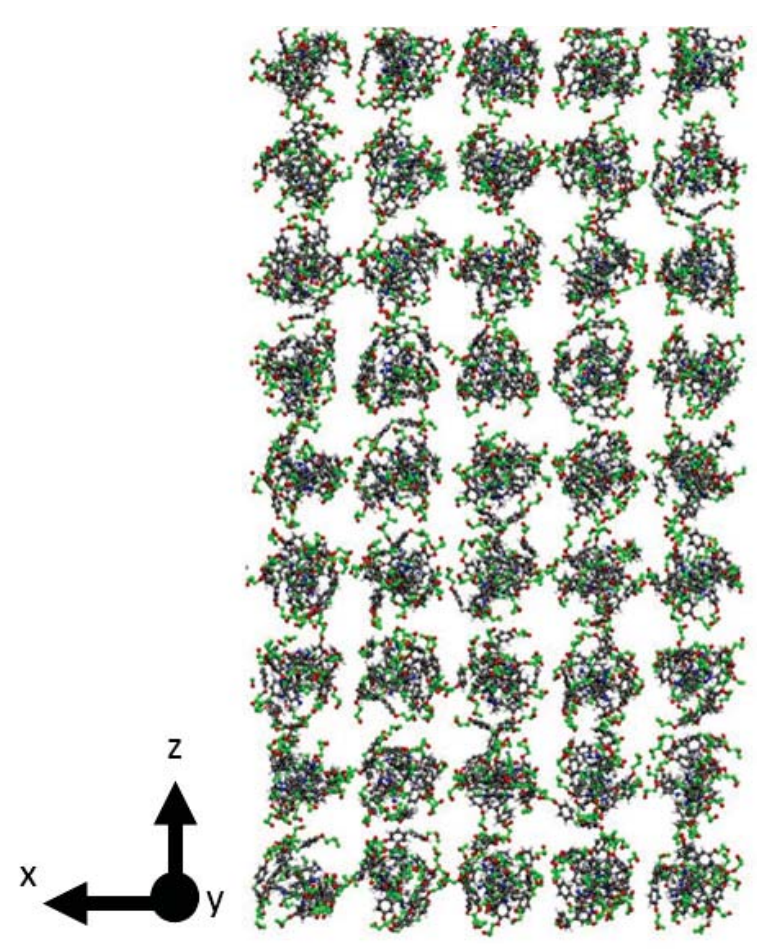

Figure 3.1 Resulting structure after implementing the 'randomly rotate and translate' program for multiplying the initial 2:1 ratio structure.

For the creation of the graphite surface, a program was written to create sheets of carbon based on the aromatic pattern for which graphite surfaces are well known. By assigning atom IDs in a specific pattern, sections of code were easily written to identify the appropriate bonds, angles, and dihedrals for a given sheet of aromatic carbon geometry. The simulated graphite surface was constructed from 3 sheets of stacked graphene, each sheet containing 1,728 carbon atoms for a total of 5,184 atoms. The graphene sheets were oriented along the $\mathrm{x}-\mathrm{y}$ plane, with periodic boundary conditions in the $\mathrm{x}$ and $\mathrm{y}$-direction, and had an interlayer spacing of $3.35 \AA$. Sheets were initially planar and contained no terminal hydrogens, surface imperfections, nor surface treatments. The graphite structures were relaxed using a series of MM and MD simulations, similar to that described above for the polymer structure. This initial equilibration step was performed without the presence of the polymer molecules. While equilibrating the graphite, the $\mathrm{z}$-direction box coordinate was chosen to implement interlayer spacing for periodic boundary conditions. Thus the top surface was influenced by the bottom surface and visa-versa, representing 
many layers of bulk graphite. Initial and relaxed graphite structures are shown in Figure 3.2. Upon relaxation, the graphite structure was positioned to line up with the 64 x $66 \AA$ dimension of the polymer structure.

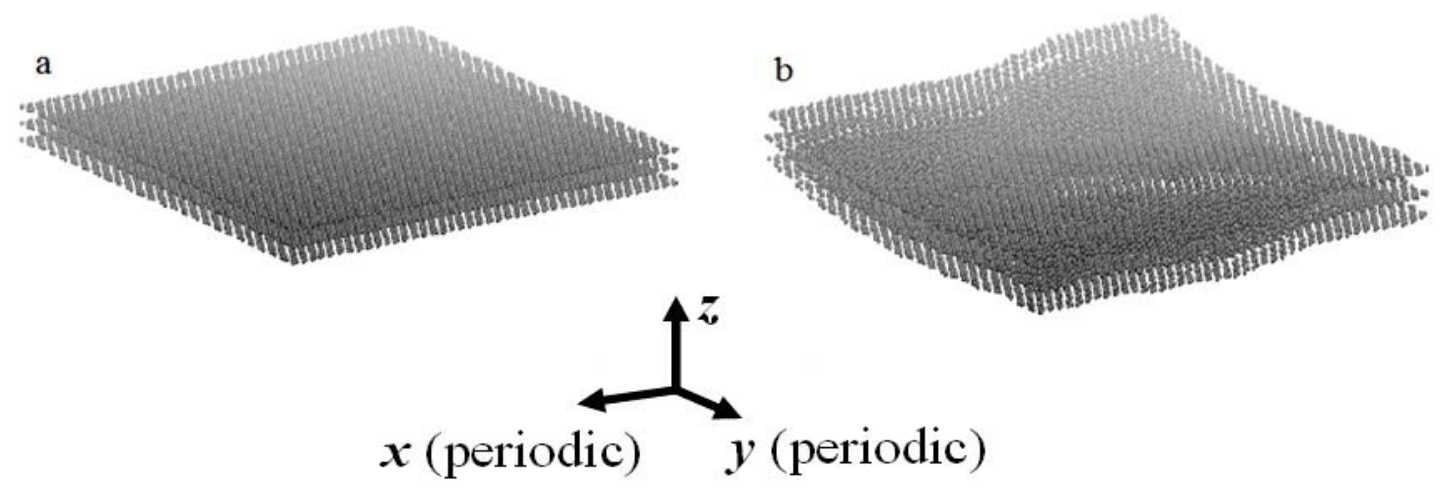

Figure 3.2 a) Initial planar graphite structure and b) Graphite structure after equilibration

For the next step in the procedure it was desired to simulate the noncrosslinked, low-density epoxy liquid resin in a parallelepiped simulation box surrounded on the bottom side (lower z-coordinate) by the graphite structure, on the opposite side (upper z-coordinate) by the bulk epoxy resin, and on the remaining four sides ( $\mathrm{x}$ and $\mathrm{y}$ ) by replicate images of the low-density liquid resin. This is shown on the left side of Figure 3.3 where the simulated polymer (labeled as "polymer") is situated above the graphite molecules along the z-axis and below a bulk polymer molecular structure. While the graphite and low-density polymer structures were established as described above, the bulk polymer structure is a fully crosslinked model of $76 \%$ crosslinked EPON 862/DETDA resin with a bulk density of $1.2 \mathrm{~g} / \mathrm{cc}$ and 17,928 united atoms that was obtained previously by fellow researcher, A. Bandyophadyay. ${ }^{20}$ Periodic boundary conditions were assumed along the $\mathrm{x}$ - and $\mathrm{y}$ axes. Therefore, the molecular model effectively simulated an infinitely large, flat interface. Although fiber surfaces are round, the radius of curvature at the molecular level is large enough to effectively model it as a flat surface. An initial gap of $3.35 \AA$ (interlayer spacing of graphite) was placed between the graphite and polymer. The entire molecular model was then allowed to partially equilibrate using two MM simulations and one 100 ps MD simulation, so that molecules from each individual 
structure (polymer, graphite, bulk) could react to one another The entire model contained a total of 39,048 united atoms.

Following this initial equilibration step, the atoms of the bulk polymer and the graphite sheets were fixed (no movement) and only non-bonded interactions between them and the polymer were considered for the remaining simulation processes. This step was performed to reduce computation time and to focus the simulations on the influence of the presence of the graphite sheet on the adjacent polymer molecular structure.

During the next step of establishing the composite interface molecular model, the simulated polymer molecules were condensed to achieve the desired polymer density. The non-bonded van der Waals parameters were initially scaled down to prevent large increases in the energy of the system. In order to reach the appropriate polymer density, the atoms of the bulk polymer were displaced in small incremental amounts using the following equation,

$z_{i}^{\text {new }}=z_{i}^{\text {current }}-S\left(z_{i}^{\text {current }}-z^{\text {bottom }}\right)$

where $z_{i}^{\text {new }}$ is the new $\mathrm{z}$-coordinate for polymer atom $i, z_{i}^{\text {current }}$ is the current $\mathrm{z}$ coordinate of polymer atom $i, z^{\text {bottom }}$ is the lower-most polymer atom z-coordinate, and $S$ is a scaling factor for the amount of displacement desired. For this study, $S$ varied between 0.02 and 0.05 , becoming smaller as the system became closer to the target density and the non-bonded van der Waals parameters were gradually scaled up to normal values. Under this constraint, the polymer atom with the lowest $\mathrm{z}$ coordinate value (nearest to the graphite) was not displaced and the polymer atom with the highest z-coordinate (nearest to the bulk polymer) was displaced the most for each incremental step. The bulk polymer molecules were uniformly displaced the exact amount as the polymer atom with the highest $\mathrm{z}$-coordinate, thereby continually condensing the polymer atoms. Each displacement was followed by a MM minimization and a 50ps MD simulation, followed lastly by another MM minimization. If a particular displacement step resulted in total energy values that 
were relatively high, the previous equilibration step was repeated before further condensation steps were taken. This process continued until achieving a polymer density of $1.16 \mathrm{~g} / \mathrm{cc}$, totaling over $1 \mathrm{~ns}$ of MD simulation time. The resulting molecular model is shown on the right side of Figure 3.3. It should be noted here that this initial condensation procedure was implanted before the discovery of the fix/deform command in the LAMMPS software, which allows the user to deform the simulation box along any specified axis during an MD simulation. The fix/deform command was implemented in later models.

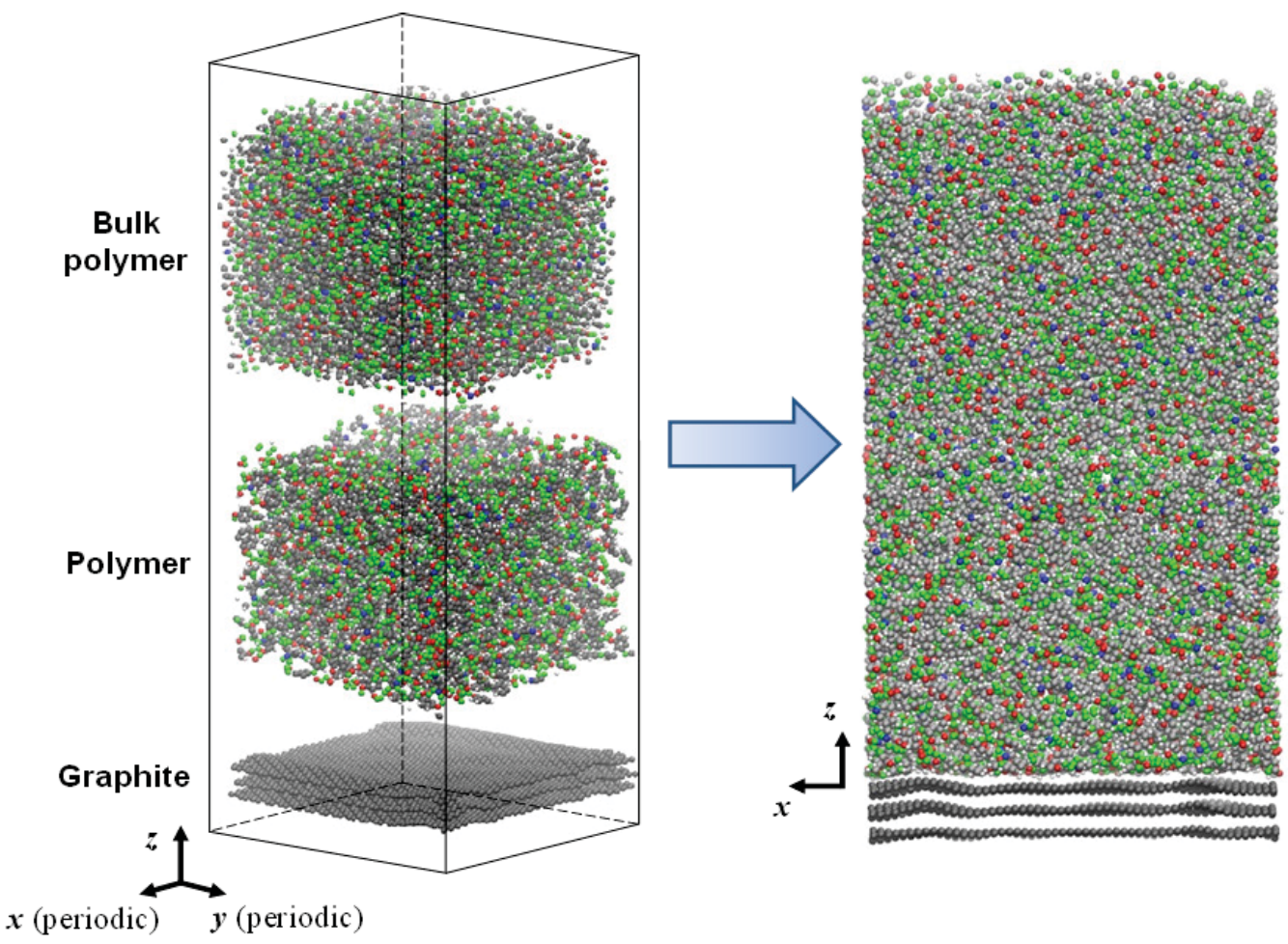

Figure 3.3 Combining the three separate MD models (left) to form the complete MD model of the composite interface (right).

After condensing the model to the desired density, crosslinking was performed using the method described in section 2.3 (page 12). A total of six molecular systems were established, each having a unique crosslink density. The chosen crosslink densities were 57\%, 65\%, 70\%, 75\%, 80\%, and 85\%. After crosslinking to the desired density, each structure was allowed to equilibrate using 3 MM minimizations and 2 MD simulations of 1 nanosecond. The density of formed 
crosslink atoms (C-N and $\mathrm{O}-\mathrm{H})$ as a function of the $\mathrm{z}$-axis can be seen in Figure 3.4, where the origin of the z-axis lies in the center of the top graphene sheet, as shown on the right side of Figure 3.3. The figure shows good dispersion of crosslinks throughout the polymer structure with unique profiles for each crosslink density. Therefore, the crosslink distributions are relatively uniform and independent of crosslink density. All density calculations were performed using the fix/ave spatial command in the LAMMPS software. ${ }^{12}$

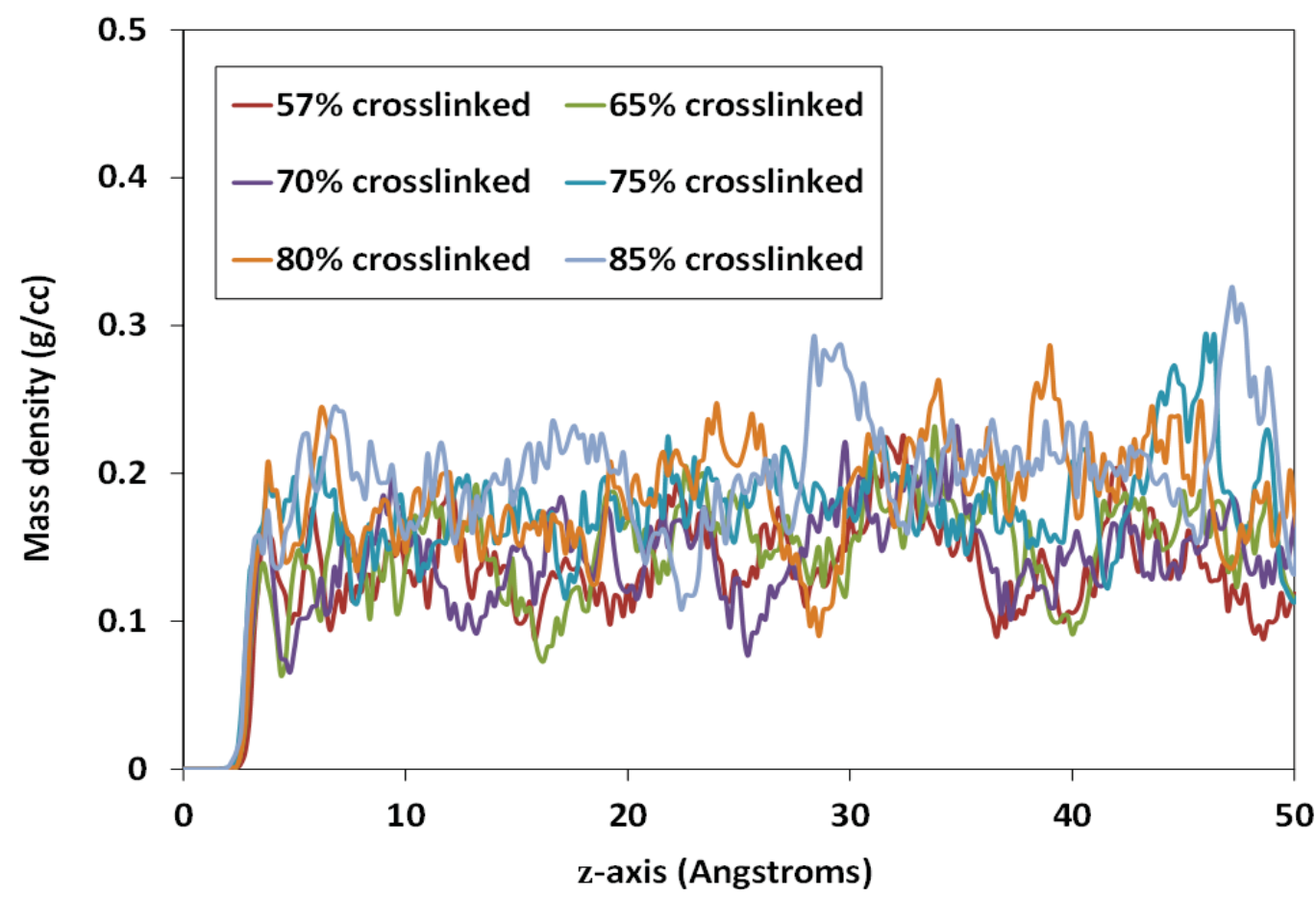

Figure 3.4 Crosslink atom mass density along the z-axis for each crosslinked structure. Z-axis origin is taken from the center of mass of the closest graphene sheet.

\subsection{Results and Discussion}

After the models were equilibrated, they were used to examine the effects of crosslink density on the overall polymer mass density at the polymer-graphite 
interface, the internal stress concentrations in the polymer at the interface, and the different potential energy components in the polymer.

\section{Polymer Mass Density Characteristics}

Mass densities were computed using the "fix/ave" spatial command in LAMMPS $^{12}$, which sums the per-atom densities and averages them for slices of a specified thickness along a specified axis. A slice thickness of $0.2 \AA$ stacking along the $\mathrm{z}$-axis was chosen to observe the structural change moving away from graphite surface oriented in the $x-y$ plane. Figure 3.5 shows the overall mass density profile for the three graphite sheets and polymer molecules at the interface. The finite width of the graphite sheet peaks is due to their slightly non-planar shape (Figure 2). The mass density of the polymer near the surface differs from that in the bulk with a profile similar to those reported for polymer systems with graphite 27,33 , carbon nanotube ${ }^{25}$ and nanoparticle ${ }^{29,30}$ reinforcement. Common interfacial characteristics include (moving along the positive z-axis) an initial peak above bulk mass density, followed by a trough below bulk density, ending with a small peak above bulk density before leveling off.

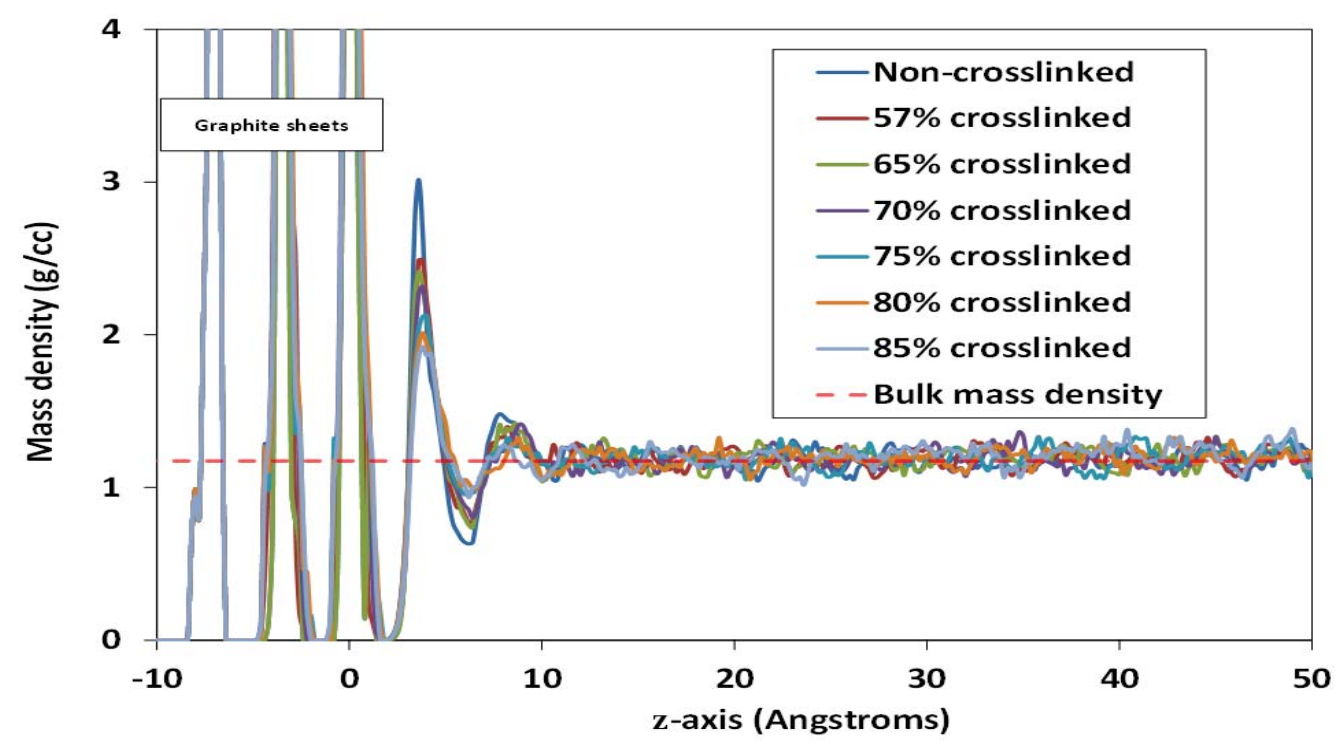

Figure 3.5 Mass density profile along Z-axis of simulation box for graphite and polymer molecules. Z-axis zer value is taken as the center of mass for the outermost graphite layer. Polymer bulk density is shown by the red line $(\sim 1.16 \mathrm{~g} / \mathrm{cc})$. 


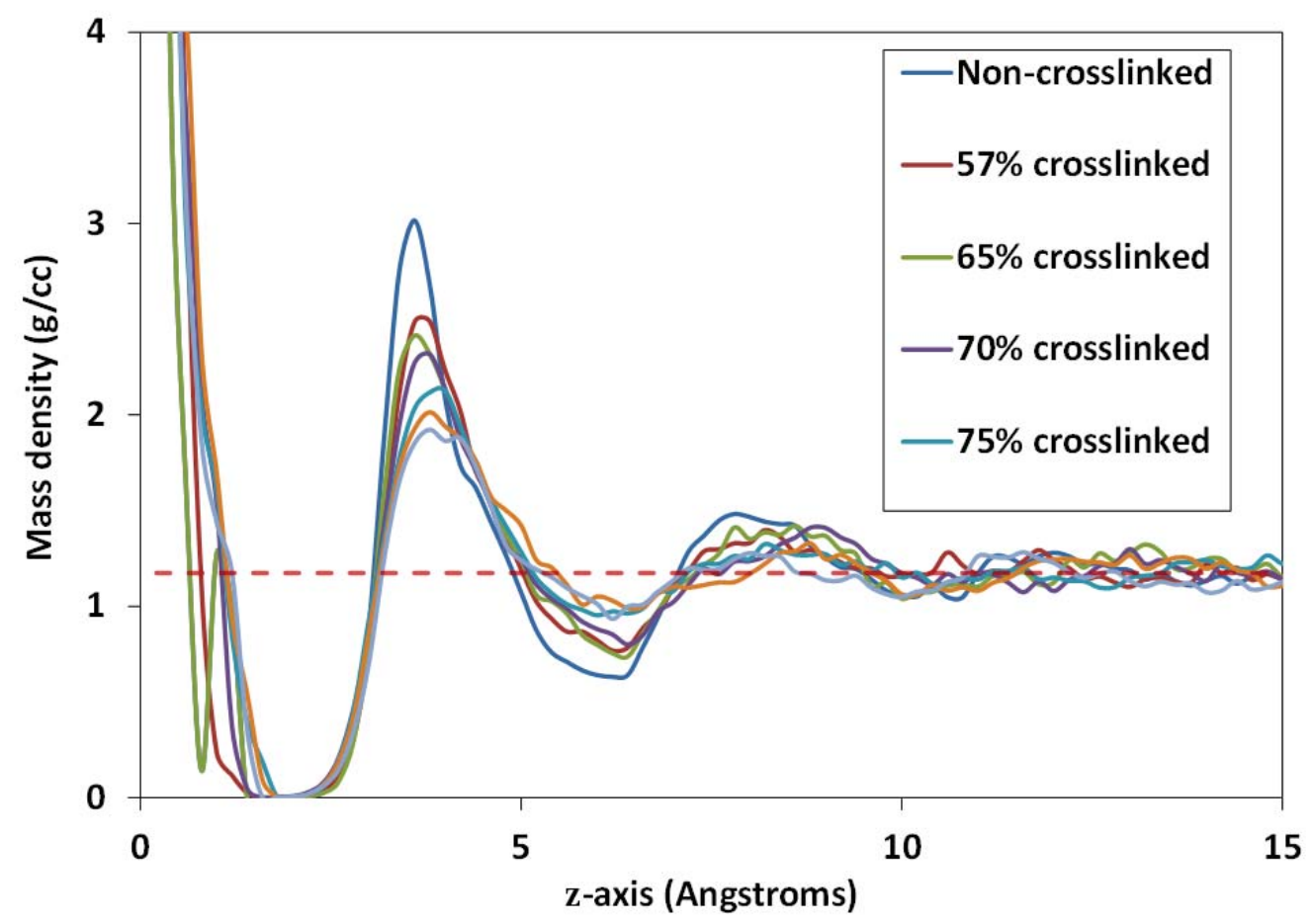

Figure 3.6 Interfacial mass density profile along Z-axis of simulation box (zoomed in from Figure $3.5)$

Figure 3.6 shows the same data shown in Figure 3.5, but focused on the interfacial region within $15 \AA$ of the graphene sheet. The fluctuation in mass density is observed for about $10 \AA$ from the center of the nearest graphite sheet. Therefore, the effective surface thickness of a polymer in the vicinity of graphite is about $10 \AA$. Figure 3.6 also demonstrates that the surface effects are reduced with increasing crosslink densities. As the polymer approaches higher crosslink densities, the mass density peak amplitudes reduce and the structure more closely resembles the bulk mass density distribution. This behavior is most likely due to the tendency of the crosslinks to hold the network together in a more spatially consistent manner.

The predictions shown in Figures 3.5 and 3.6 are supported by the transmission electron microscopy (TEM) image shown in Figure 3.7. The image shows a portion of an open-ended carbon nanofiber reinforcing a crosslinked epoxy matrix. The surface of the carbon nanofiber and the structure of the epoxy system are similar to those modeled herein. The bulk epoxy matrix is amorphous; however, a small band of 
structured epoxy can be seen on the surface of the nanofiber, as indicated in the figure. Although the thickness of this interface appears to vary in the image, the magnitude of the thickness is close to the predicted value, $10 \AA$. This favorable comparison serves as a validation of the modeling strategy described herein.

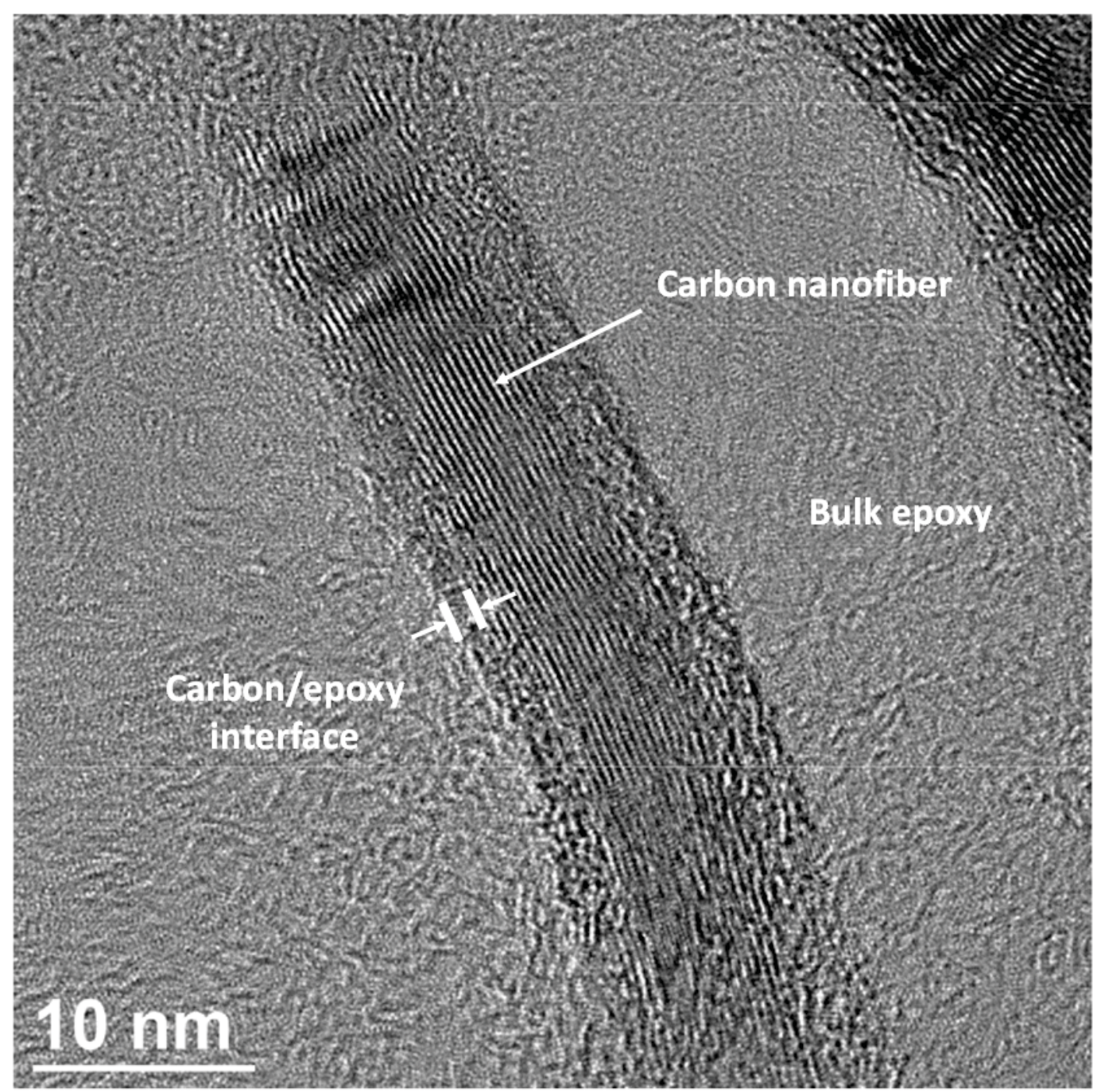

Figure 3.7 TEM image of carbon nanofiber embedded in a crosslinked epoxy resin. Image courtesy of Z. Liang et. al, High-Performance Materials Institute, Florida State University 
Figure 3.8 shows the mass density profiles for the EPON 862 and DETDA molecules for the non-crosslinked and $80 \%$ crosslinked systems. In general, the densities of EPON 862 are larger than those of DETDA, which is mostly because there are two EPON 862 molecules for every DETDA molecule. For both levels of crosslinking, larger concentrations of EPON are observed near the surface. Large concentrations of DETDA are only present at the surface for the non-crosslinked system. Therefore, before crosslinking occurs, both molecules are concentrated at the surface. During the process of crosslinking, the concentrated DETDA molecules are pulled away from the surface so that the DETDA mass density profile is relatively uniform.

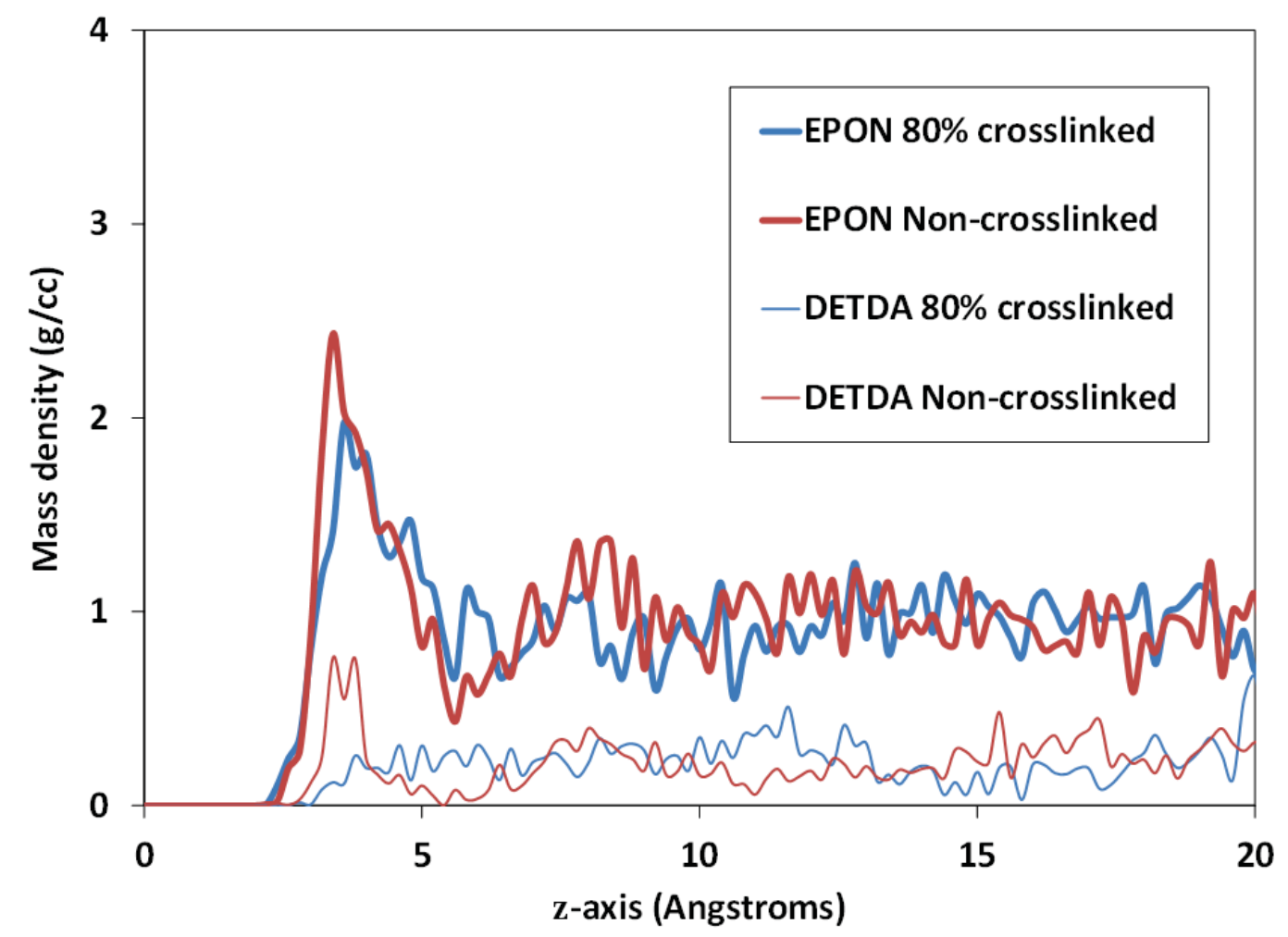

Figure 3.8 EPON 862 and DETDA molecular mass densities along simulation box Z-axis for Noncrosslinked and $80 \%$ crosslinked structures.

\section{Polymer Internal Stress Characteristics}

Stress components were computed using the LAMMPS "fix/ave" spatial command in a similar manner as described above for the mass density profiles. Each 
normal and shear stress component was computed for each individual atom and averaged in slices of $0.1 \AA$ along the $\mathrm{z}$-axis of the simulation box. The individual stress components were then used to calculate the Von Mises stress using the following equation:

$\sigma_{V M}=\frac{1}{\sqrt{2}} \sqrt{\left(\sigma_{x}-\sigma_{y}\right)^{2}+\left(\sigma_{y}-\sigma_{z}\right)^{2}+\left(\sigma_{z}-\sigma_{x}\right)^{2}+6\left(\tau_{x y}^{2}+\tau_{y z}^{2}+\tau_{z x}^{2}\right)}$

The Von Mises stress along the z-axis for a range of crosslink densities is shown in Figure 3.9. The stress peak nearest to the origin is due to stresses in the graphite sheet. Residual stresses are observed for each of the crosslink densities with the largest values at the interface. Despite the large amount of scatter in the data, there appears to be no influence of the crosslink density on the location and magnitude of residual stresses. The largest amount of stress in the polymer is considerably low for all crosslink densities, well below the expected tensile strength of EPON-862, which is $70-95 \mathrm{MPa}$ at room temperature. ${ }^{48}$ The presence of a small concentration of residual stress near the interface indicates that upon significant loading of an epoxy/graphite composite, failure of the resin matrix would likely occur first near the fiber/matrix interface.

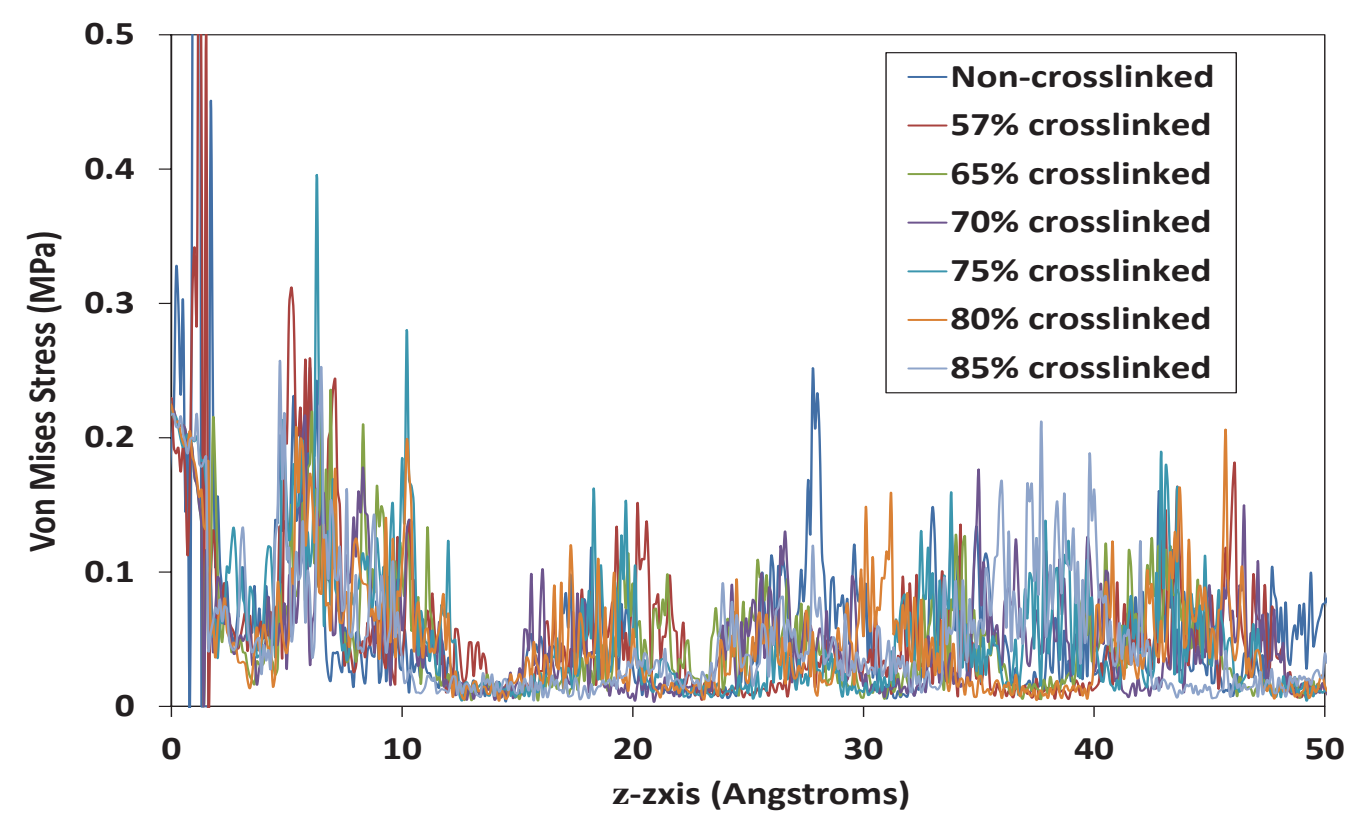

Figure 3.9 Spatially averaged Von Mises stress for polymer and graphite atoms along Z-axis. 


\section{Polymer Potential Energy Characterisitics}

Potential energy values for bonds, angles, dihedrals, and non-bonded interactions were computed with LAMMPS using the same fix/ave spatial command previously described. The bin size was $0.1 \AA$ along the z-axis. Figure 3.10 shows the total potential energy (the sum of the individual energy terms) for each system. There appears to be an increased level of potential energy near the fiber/matrix interface, which is consistent with the increase in stress at the interface shown in Figure 11. Also, the potential energy generally decreases with increasing crosslink density. The largest contributing factor to the total potential energy was found to be angle energy, which decreases with increasing crosslink density. This is likely due to the elimination of the high-energy epoxide rings on the EPON 862 molecule during the crosslinking reaction. The decreases in potential energy with increasing crosslink density shown in Figure 12 indicate that crosslinking is energetically favorable for the modeled system and force field.

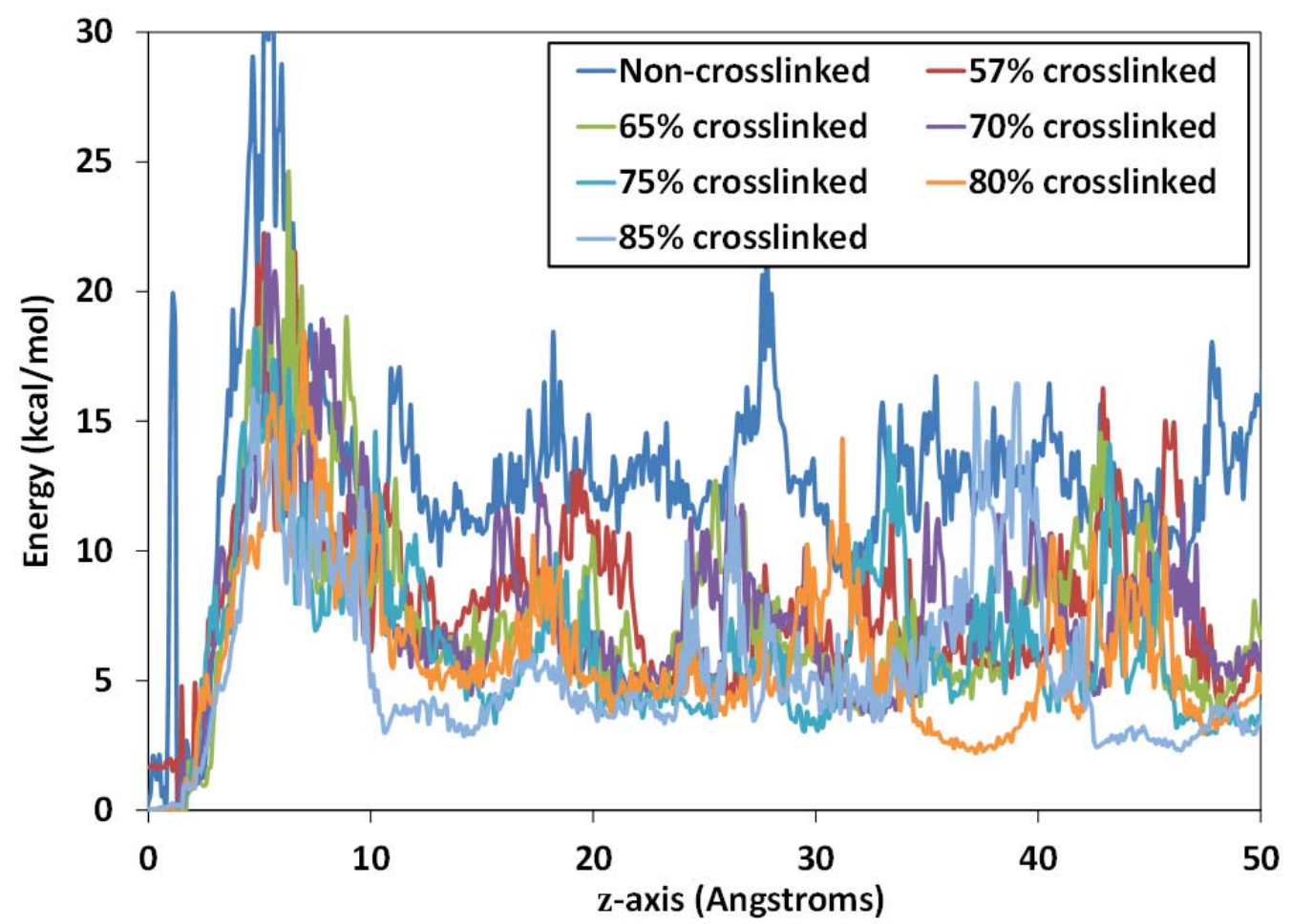

Figure 3.10 Spatially averaged per-atom potential energy. Potential energy values are the sum of bond, angle, dihedral, and non-bonded van der Waal's energies. 


\subsection{Conclusions}

In this chapter, the influence of crosslink density on the molecular structure of the graphite fiber/epoxy matrix interface was examined using MD techniques. The modeled epoxy resin was an EPON 862/DETDA system. It was determined that the mass density of the polymer within $10 \AA$ of the center of the nearest graphene sheet was perturbed from the bulk level of $1.17 \mathrm{~g} / \mathrm{cc}$. This observation agrees with predictions in the literature and our own TEM images (Figure 3.8). Although the amount of crosslink density did not influence this effective surface thickness of the epoxy near the graphite interface, increasing levels of crosslinking reduced the magnitude of mass density fluctuations within the effective surface thickness. Analysis of the mass density of polymer molecules also reveals that before crosslinking, the presence of surface effects is due to both the perturbed mass densities of the EPON 862 monomer and DETDA hardener molecules in the surface region. Local Von Mises stresses were determined at the interface of the composite, and the MD simulations demonstrated that the internal stresses were slightly higher in the surface region than in the bulk epoxy, albeit at levels far below the ultimate strength of the neat resin. The internal stresses near the interface are not influenced by crosslink density. Finally, the MD simulations predict elevated levels of molecular potential energy in the surface region of the polymer, with the potential energy magnitude decreasing with increasing crosslink levels. It can be said, with confidence, that the methods described in this

chapter are sufficient for producing accurate models of EPON 862-DETDA epoxy in the presence of a graphite surface. 


\section{CHAPTER 4: MULTI-SCALE MODELING OF GRAPHENE DOPED EPOXY ${ }^{1}$}

Chapter 4 utlizes MD to investigate the effects of graphene thickness along with varying cross-link density on the molecular scale mechanical properties. The properties derived from MD are then implemented into a Micromechanical Analysis Code with the Generalized Method of Cells (MAC/GMC) software for comparison to larger scale experimental results for materials similar to the models.

\subsection{Introduction}

The use of graphene nano-platelets (GNPs) within the epoxy matrix has been shown to enhance both mechanical and electrical properties within the composite. The effects of GNPs on the composite is governed by the amount of GNPs added to the matrix component and the dispersion of the GNPs within the matrix ${ }^{9-10,49-50}$. The interface between GNP and surrounding epoxy matrix is difficult to characterize with experimental techniques, and MD can provide valuable insight to the material behavior at the molecular level. MD can also provide information for GNPs of different sizes (atomic thicknesses), as GNPs vary significantly in size depending on fabrication techniques.

Recently, Chunyu Li. et. al. utilized MD techniques to model a multi-layer graphene epoxy composites. Graphene orientation was investigated, though the number of atomic layers and crosslink density of the polymer remained constant. Mechanical properties were also derived, though no experimental comparison was incorporated ${ }^{37}$. Jing-Suei Gao et. al. investigate the mechanical properties of polyimide polymer near a graphene sheet of 5 atomic layers thick. Interfacial effects were

\footnotetext{
${ }^{1}$ The information from this chapter will be considered for publication in the near future.
} 
investigated and mechanical properties were derived. Again, the results did not allow for comparison to larger scale experimental data. ${ }^{36}$ Although these studies have given valuable information regarding the physical nature of the interfacial region, including an estimate of the mechanical properties on a molecular level, there has been little effort to implement this molecular data into a larger scale model that will enable comparison to experimental results for similar systems.

The purpose of this study was to use mechanical data provided by MD for epoxy/graphene systems, and implement this data into a larger scale model using the principals derived from the Generalized Method of Cells (GMC) ${ }^{51}$ MD models were constructed for a wide range of epoxy crosslink densities (curing ranges) with graphene structures of varying atomic thickness from a single atomic layer up to 4 layers thick. Mechanical properties were extracted from each model and then inserted into Micromechanics Analysis Code with Generarlized Method of Cells (MAC/GMC) software ${ }^{51-53}$ to calculate properties for an epoxy/GNP composite with varying GNP concentration and dispersion at a fixed polymer cross-link density (explained in detail in section 4.3). The results for a GNP doped epoxy matrix for a specified GNP concentration and dispersion can be re-introduced to the MAC/GMC software in the presence of a carbon fiber to yield properties for a GNP doped epoxy/carbon fiber composite. MAC/GMC results for the GNP doped epoxy matrix calculation, along with that for a GNP doped epoxy/carbon fiber composite are in good agreement with experimental data. Composite properties increase with an increase in GNP concentration. Results are best for single layered GNPs, which also represent the highest amount of dispersion in the GMC models.

\subsection{Modeling Procedures}

The initial un-crosslinked polymer model began as the 2:1 stoichiometric ratio of the EPON 862 monomer and the DETDA hardener outlined in section 2.2 (page 9). The 2:1 molecular model was subjected to four MM minimizations and 
three 100 ps MD simulations. MM simulations utilized the conjugate gradient stopping criterion, and MD simulations were performed using the NVT ensemble at $300 \mathrm{~K} .{ }^{12}$ This process minimized internal forces and thus reduced internal residual stresses which were created from the initial construction of bonds, bond angles, and bond dihedrals. After the structure stabilized to a relatively low energy value, the initial 2:1 stoichiometric structure was replicated, and the replicated models were randomly rotated and then translated along the $\mathrm{x}, \mathrm{y}$, and $\mathrm{z}$ axes and combined into a much larger structure with an EPON 862:DETDA ratio of 250:125, containing 10,375 total united atoms. Therefore, the resulting system consisted of 250 randomly oriented clusters of the small 2:1 ratio cluster stacked loosely together in a manner much like that of a simple cubic crystal structure. This larger polymer model was then mirrored about a graphene structure positioned in the $\mathrm{x}-\mathrm{y}$ plane central to the z-axis. As a result, each system contains a 500:250 ratio of EPON 862:DETDA totaling 20,750 polymer atoms. The centralized graphene structures varied in atomic thickness from 1 atomic layer up to 4 layers thick, each layer containing 4200 carbon atoms. The largest system, comprised of a 4 layer graphene sheet, contained 37,550 total atoms and the initial box size was $101 \times 104$ x $210 \AA$. All models employed 3D periodic boundary conditions, which is necessary for deformation simulations. The initial box size produced a polymer density approximately equal to half of a fully cured solid EPON 862 epoxy ( $\sim .5 \mathrm{~g} / \mathrm{cc}$ in all 4 systems). Unlike the previous model, the mirrored epoxy structures and the graphene structures were all combined in one step and allowed to equilibrate in the presence of one another for $200 \mathrm{ps.} \mathrm{In} \mathrm{order} \mathrm{to}$ achieve the desired polymer density of $1.17 \mathrm{~g} / \mathrm{cc}$, the 4 separate models were subjected to 12 cycles of deformation along the z-axis. Each cycle included a MM followed by a 100ps MD NVT simulation in which the $\mathrm{z}$ coordinate was reduced in equal amounts from both the positive and negative z-coordinate boundaries using the LAMMPS fix/deform tool. A Nose/Hoover thermostat and barostat was implemented for temperature and pressure control, respectively. ${ }^{46}$ The amount of deformation decreased with each cycle as the models became closer to the desired density. This was done to avoid large energy increases to the system by packing the 
molecules together too quickly. This entire densification process was done over a total of $1.2 \mathrm{~ns}$ for each of the 4 systems. The final z-coordinate boundary enabled for polymer atoms to extend $\sim 13 \AA$ from the graphene surface, to ensure that the interfacial region was fully captured, and show a minimal influence from the bulk polymer characteristics during deformation. A visual representation for the densification process can been seen for the single graphene sheet system in Figure 4.1. Fully equilibrated, non crosslinked, structures for all 4 systems can be seen in Figure 4.2.

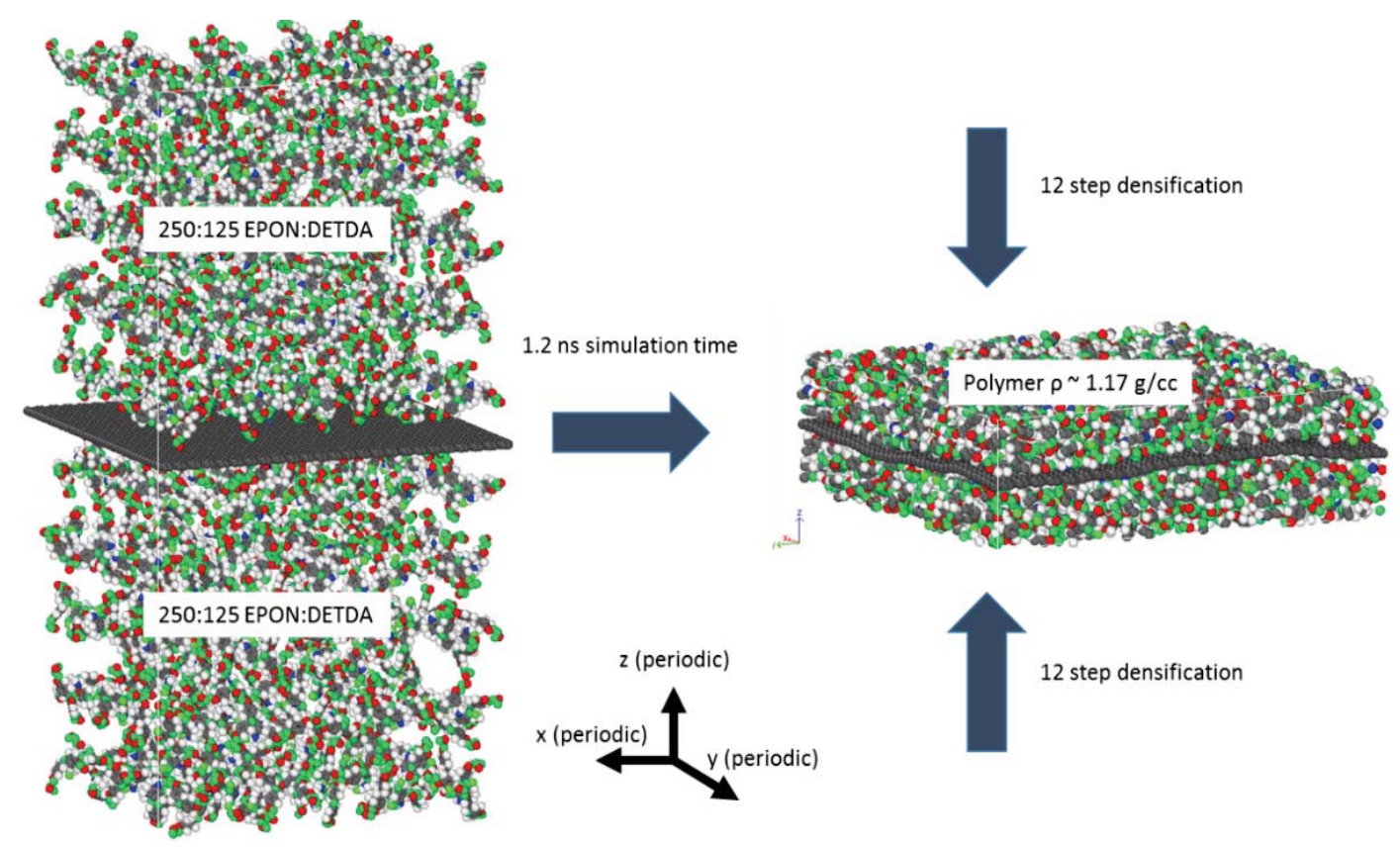

Figure 4.1 Molecular structures for single layer graphene sheet model highlighting the densification process. 


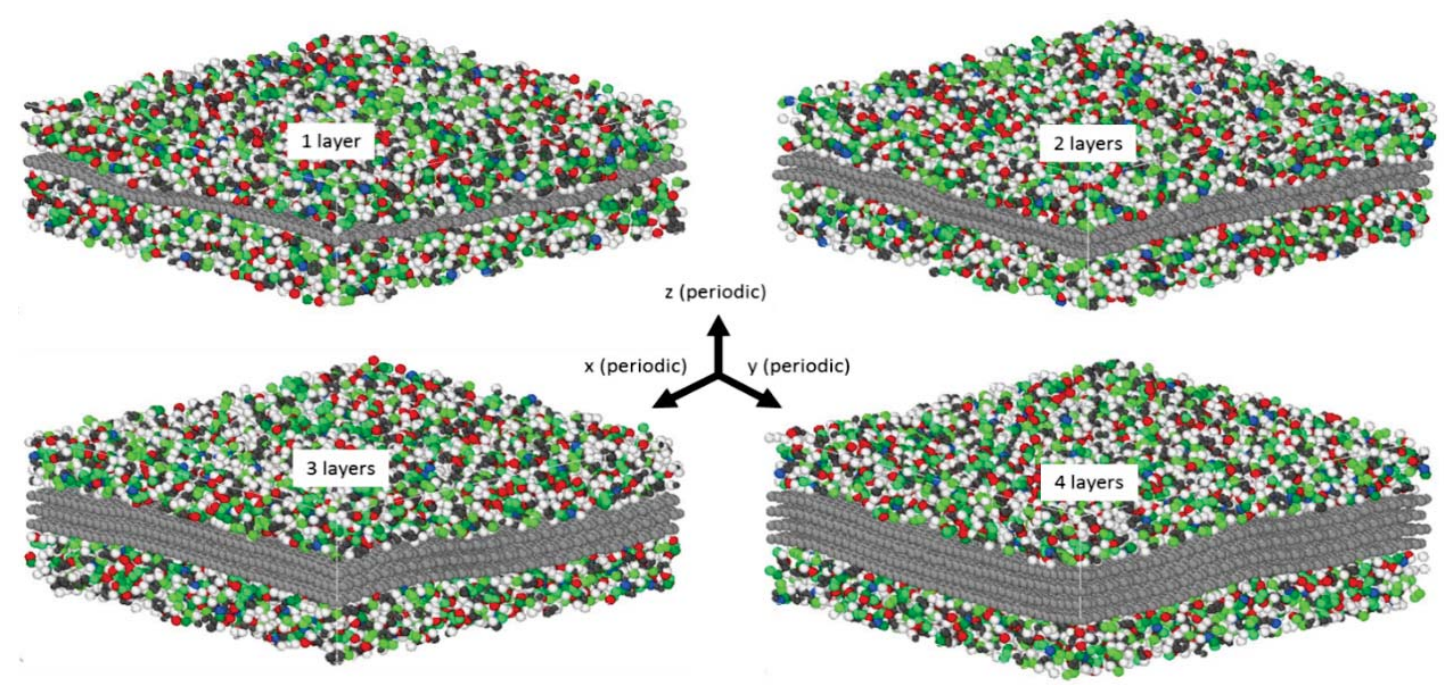

Figure 4.2 Equilibrated models for varying number of graphene layers before crosslinking. Polymer density $\sim 1.17 \mathrm{~g} / \mathrm{cc}$ for each model.

After condensing the models to the desired density, crosslinking was performed as previously described in section 2.3. For this study, multiple crosslink densities were considered in conjunction with varying graphene thickness. The chosen crosslink densities were $65 \%, 70 \%, 75 \%$, and $80 \%$. Figure 4.3 shows the density profile along the z-axis for the $80 \%$ crosslinked models. Again, there is good dispersion of crosslinks throughout the models with unique profiles for each model with a varying number of graphene atomic layers. Crosslink density profiles for $65 \%$, $70 \%$, and $75 \%$ produce similar results. 


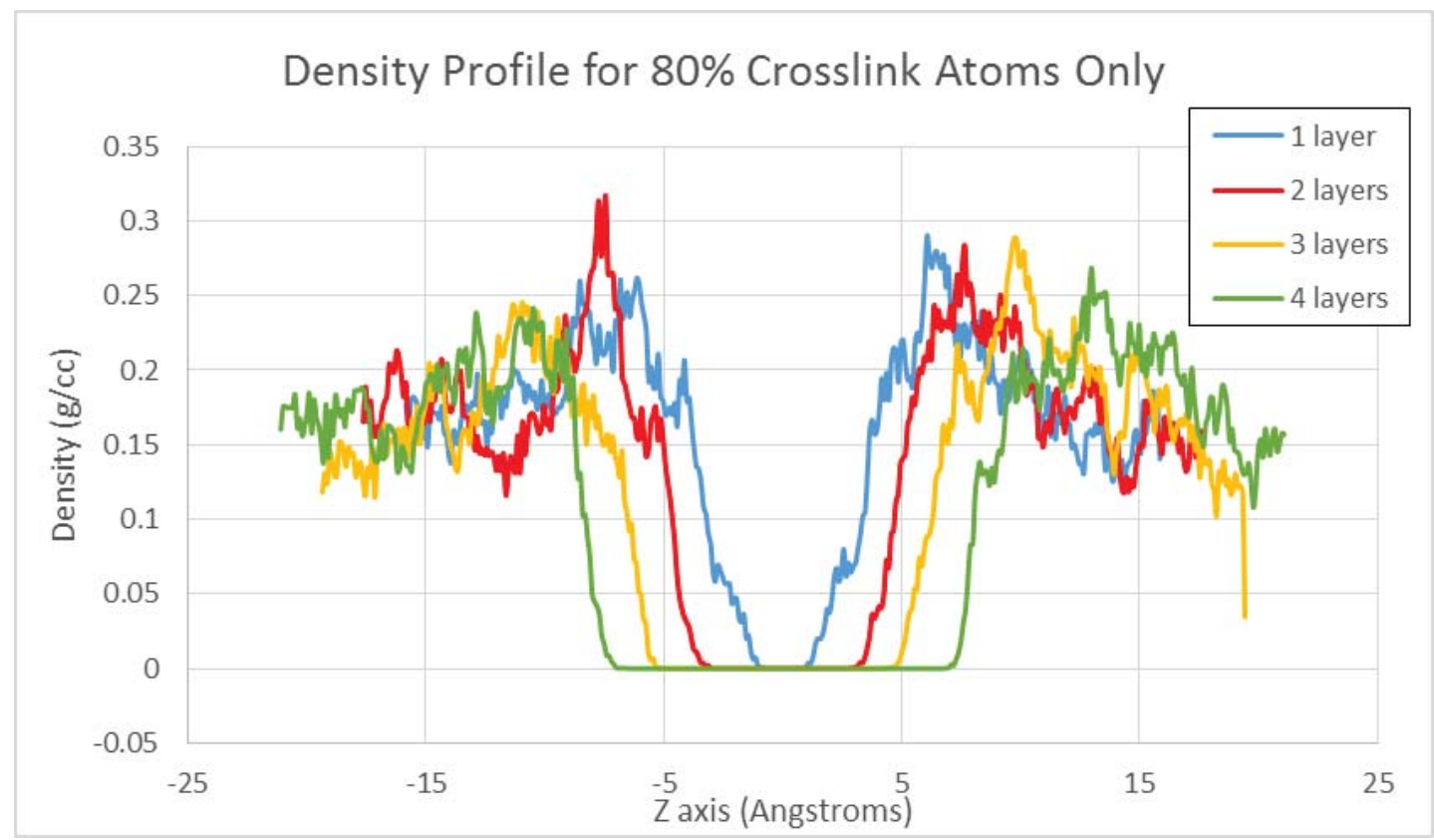

Figure 4.3 Crosslink dispersion as a function of $\mathrm{z}$-axis coordinate for $80 \%$ crosslinked systems.

\section{Mechanical Deformation}

Molecular models were assumed to be transversely isotropic in the $\mathrm{x}$ and $\mathrm{y}$ directions. In order to develop Stiffness matrices for each of the 16 systems, models were deformed in both tension and shear. Tensile deformations of 5\% were performed separately along the $\mathrm{x}, \mathrm{y}$, and $\mathrm{z}$ axes over the period of $1 \mathrm{~ns}$ for each model. Tensile deformations aloud for Poisson's contractions in the transverse directions for calculation of Poisson's ratio. In order to accomplish this, it is necessary to utilize the NPT ensemble to allow for volume adjustments. Shear deformations of $5 \%$ were performed separately along the $\mathrm{x}-\mathrm{y}, \mathrm{y}-\mathrm{z}$, and $\mathrm{x}-\mathrm{z}$ plane over the period of $1 \mathrm{~ns}$ for each model. Shear deformations do not allow for contraction, and thus incorporate the NVT ensemble. A visual representation of tensile deformation along the $\mathrm{z}$-axis, and shear deformation in the $\mathrm{x}-\mathrm{z}$ direction is shown in Figure 4.4. Resulting stress vs. strain curves for tensile deformation along the $\mathrm{x}$-axis and shear in the xy-plane are shown for the $80 \%$ crosslinked models in Figure 4.5. Data shows very little scatter, indicating an acceptable strain-rate. All mechanical data derived from MD models can be seen in Table 4.2 shown later in this chapter. 

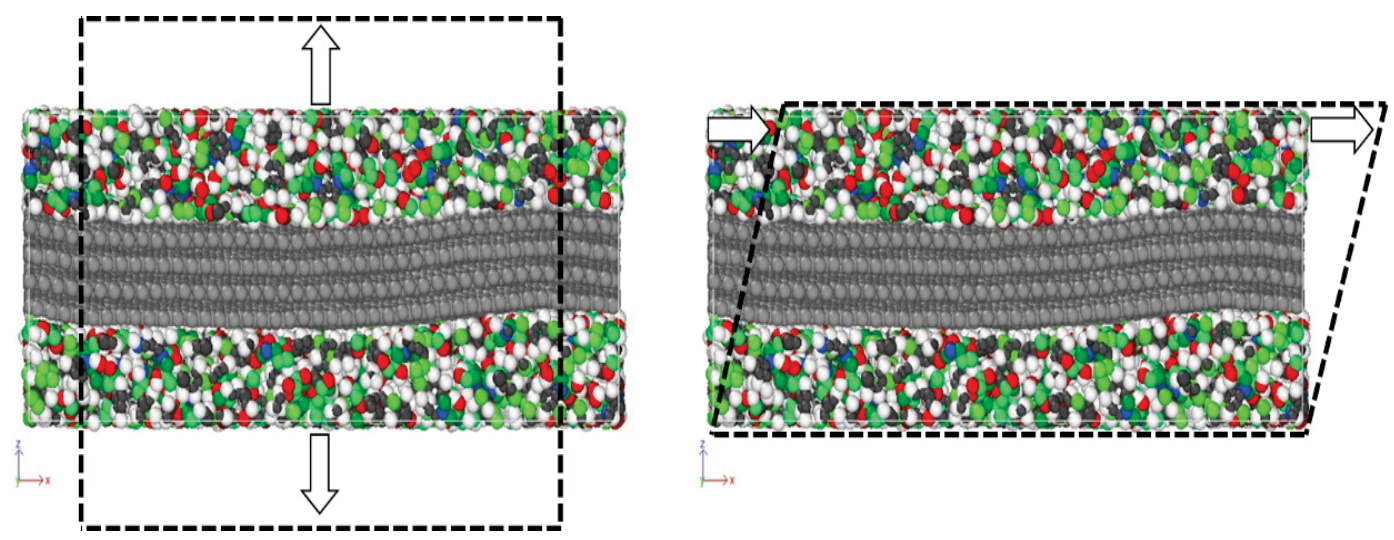

Figure 4.4 Simulated deformation characteristics for tension along the z-axis (left) and shear in the $\mathrm{x}$ $\mathrm{z}$ direction (right)

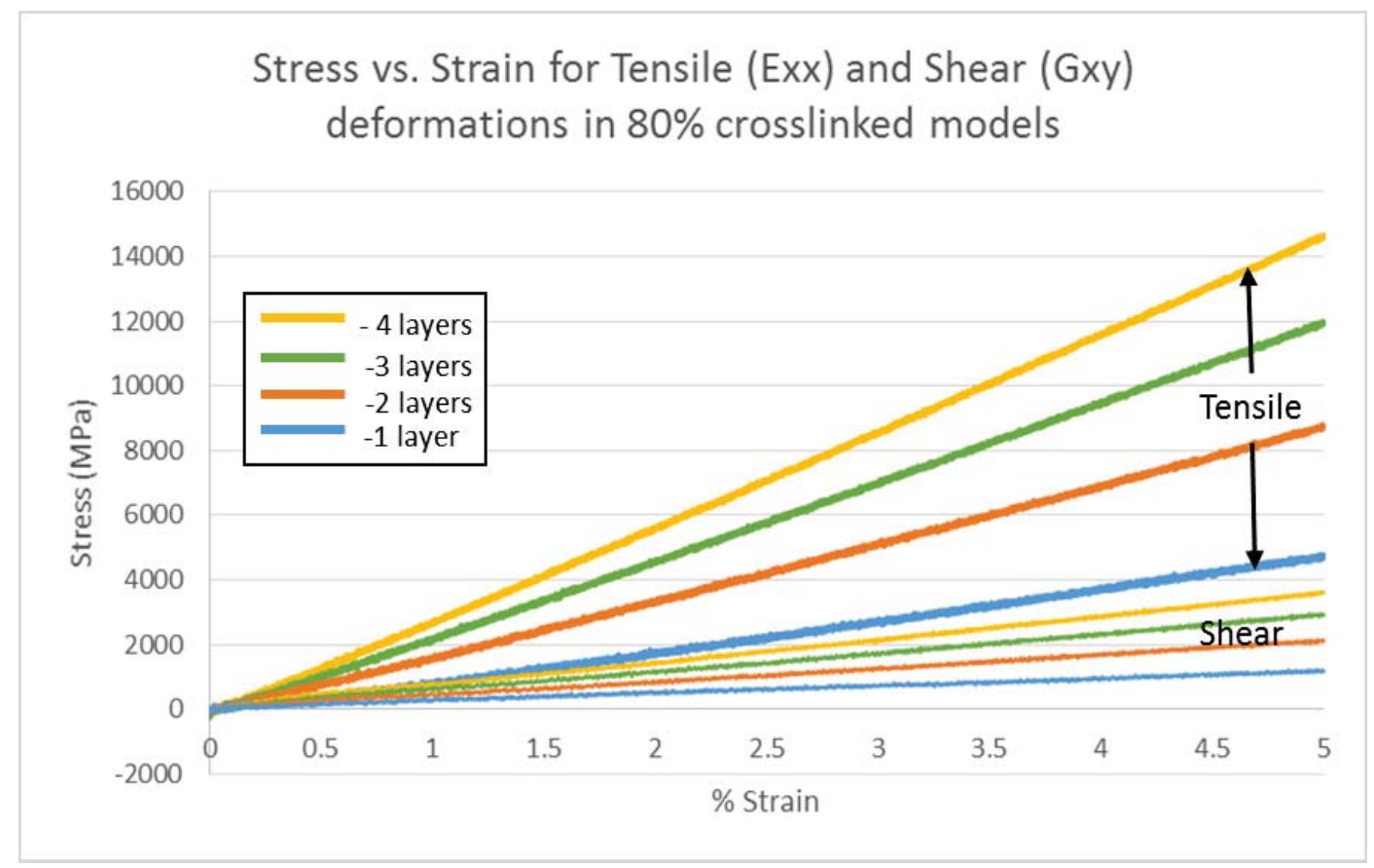

Figure 4.5 Tensile and Shear stress vs strain curves for varying number of graphene layers in $80 \%$ crosslinked structures. 


\section{Micromechanics}

Larger scale models were developed using MAC/GMC software created by Brett Bednarcyk et. al. ${ }^{51-53}$ The software utilizes the concepts of the Generalized Method of Cells (GMC) from micromechanics. ${ }^{51}$ GMC estimates macro-scale material properties by investigating a very small representative unit cell (RUC) that can be multiplied in a periodic array into a larger scale composite. The RUC commonly contains volumes representing a polymer matrix and a carbon reinforcement, where the material properties from each component determine that of the entire composite.

\section{GNP Doped Epoxy Model}

In order to develop a model for a GNP doped EPON 862 epoxy, the mechanical properties derived from each MD model were implemented into a small volume portion of the RUC. The surrounding volume in the RUC represented that of fully cured bulk EPON 862-DETDA epoxy. This 3D RUC was then randomly rotated and translated in the $\mathrm{x}, \mathrm{y}$, and $\mathrm{z}$ directions to create a large cube with many randomly oriented graphene structures, as seen in Figure 4.6. The GMC model allows for control of the graphene volume concentration for the overall composite, and the randomly oriented RUC was multiplied extensively to ensure a good representation of an isotropic GNP doped epoxy matrix. As the number of graphene atomic layers increases in the MD models, so does the graphene volume fraction for each RUC. Therefore, if the graphene volume within the RUC is kept constant, the model containing data from 1 atomic layer of graphene would have to be nearly 3 times as large as that for the model containing data from the 4 atomic layer graphene structure in order to achieve the same graphene concentration in the GMC models. Also, the graphene concentration in the GMC model is governed by the graphene volume fraction in the MD model. For the MD model with a single atomic layer of graphene the volume fraction is 0.11 , the GMC model will not be able simulate a higher composite graphene concentration than this. When thinking of the amount of GNPs within a composite, a single atomic layer of graphene can be thought to represent excellent dispersion of 
GNPs within the epoxy matrix, while the 4 atomic layer graphene structures will represent agglomerated GNPs within the matrix component. Volume fractions of graphene can be seen for each of the MD structures in Table 4.2.

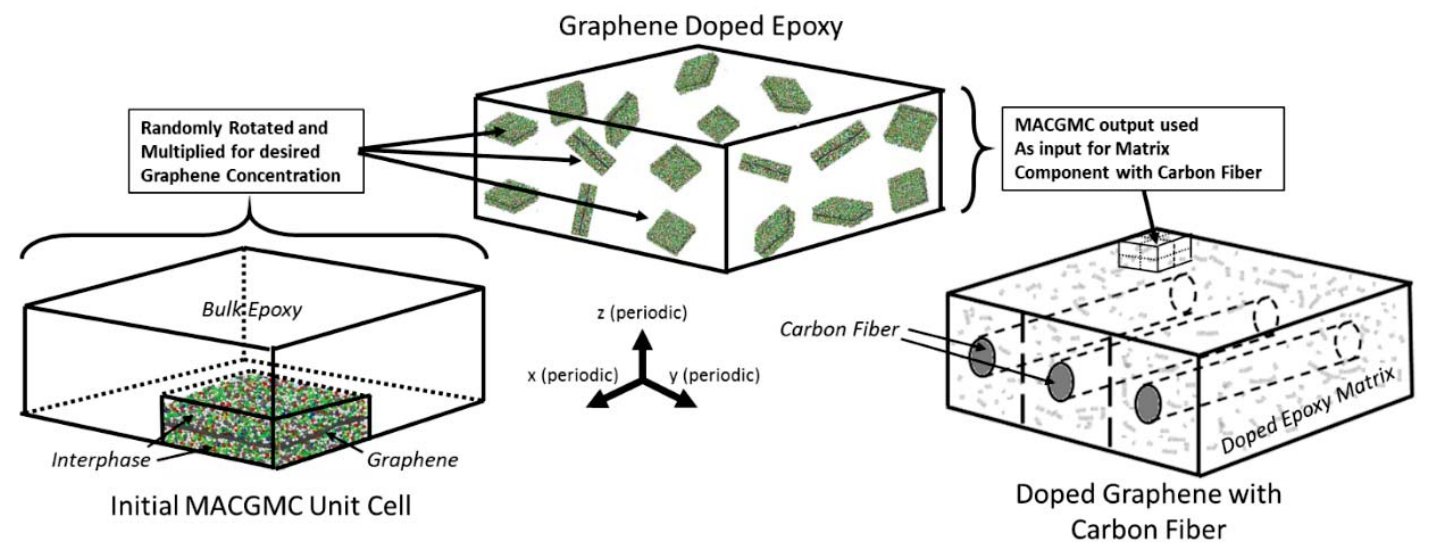

Figure 4.6 Schematic representation for implementing MD results into MACGMC software to obtain data for GNP doped epoxy and GNP epoxy/Carbon Fiber composites

\section{GNP Epoxy / Carbon Fiber Composite}

After developing micromechanics models for isotropic GNP doped epoxy specimens, the data was re-implemented into MAC/GMC in the presence of a carbon fiber. This step is shown on the right side of Figure 4.6. MAC/GMC has the ability to consider many different unit cell architectures for a carbon fiber. For this study, the fiber architecture ID (ARCHID) was chosen as a 26x26 circular array seen Figure 4.7. This figure represents the RUC used for composite data calculations. The outer portion (green) represents the GNP doped epoxy, and utilized input parameters derived from the techniques described in the previous section. The circle in the center of the RUC is the chosen geometry to represent the carbon fiber. Input parameters for the carbon fiber were chosen to accurately represent the fibers used in related experiments, and are shown in Table $4.1^{10,54}$ 
Table 4.1 MAC/GMC Input Parameters for HexTow® AS4-GP/3K Carbon Fibers

$\begin{array}{lc}\text { Tensile Modulus }\left(\mathrm{E}_{11}\right) & 231 \mathrm{GPa} \\ \text { Transverse Modulus }\left(\mathrm{E}_{22}\right) & 9.6 \mathrm{GPa} \\ \text { Shear Modulus }\left(\mathrm{G}_{12}\right) & 112 \mathrm{Gpa} \\ \text { Poisson's Ratio }\left(v_{12}\right) & 0.3 \\ \text { Fiber Volumer Fraction } & 58 \%\end{array}$

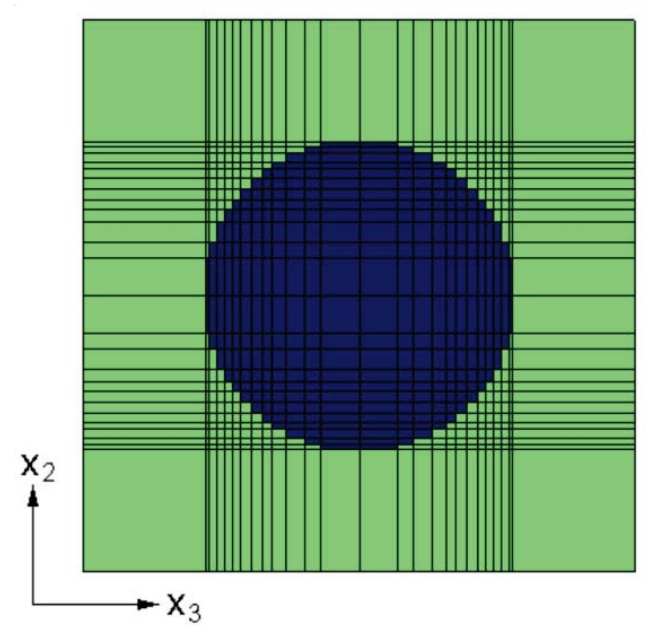

Figure 4.7 MAC/GMC RUC for GNP doped epoxy / carbon fiber model. (ARCHID = 13 for MAC/GMC version 4.0)

\subsection{Results and Discussion}

MD models were used to examine material behavior at the interface. Upon validating each model with past simulation results, MD was used to deform the simulation box and extract specific material properties. These properties were then implemented in to MAC/GMC software to estimate macroscopic material properties for both a graphene doped epoxy matrix with varying graphene concentrations, and a graphene doped epoxy matrix with unidirectional carbon fibers with volume fractions similar to experimental data. 


\section{Molecular Dynamics}

Polymer mass density profiles follow a similar trend to previous works, showing peak values near the graphene surface then fluctuating and eventually becoming stable at $\sim 10 \AA$ from the surface. Figure 4.8 shows the density vs. z-axis coordinate for varying number of graphene layers and crosslink densities. The larger spikes in the center of the density profile are from the graphene carbon atoms. These peaks are not perfectly symmetrical because the graphene is able to wave and contort during MD. Exact values for overall polymer density at the interface are given in Table 4.2. These values are $\sim .04 \mathrm{~g} / \mathrm{cc}$ less than the target value of $1.17 \mathrm{~g} / \mathrm{cc}$, likely caused by the final 1.2ns NPT relaxation simulation described above.
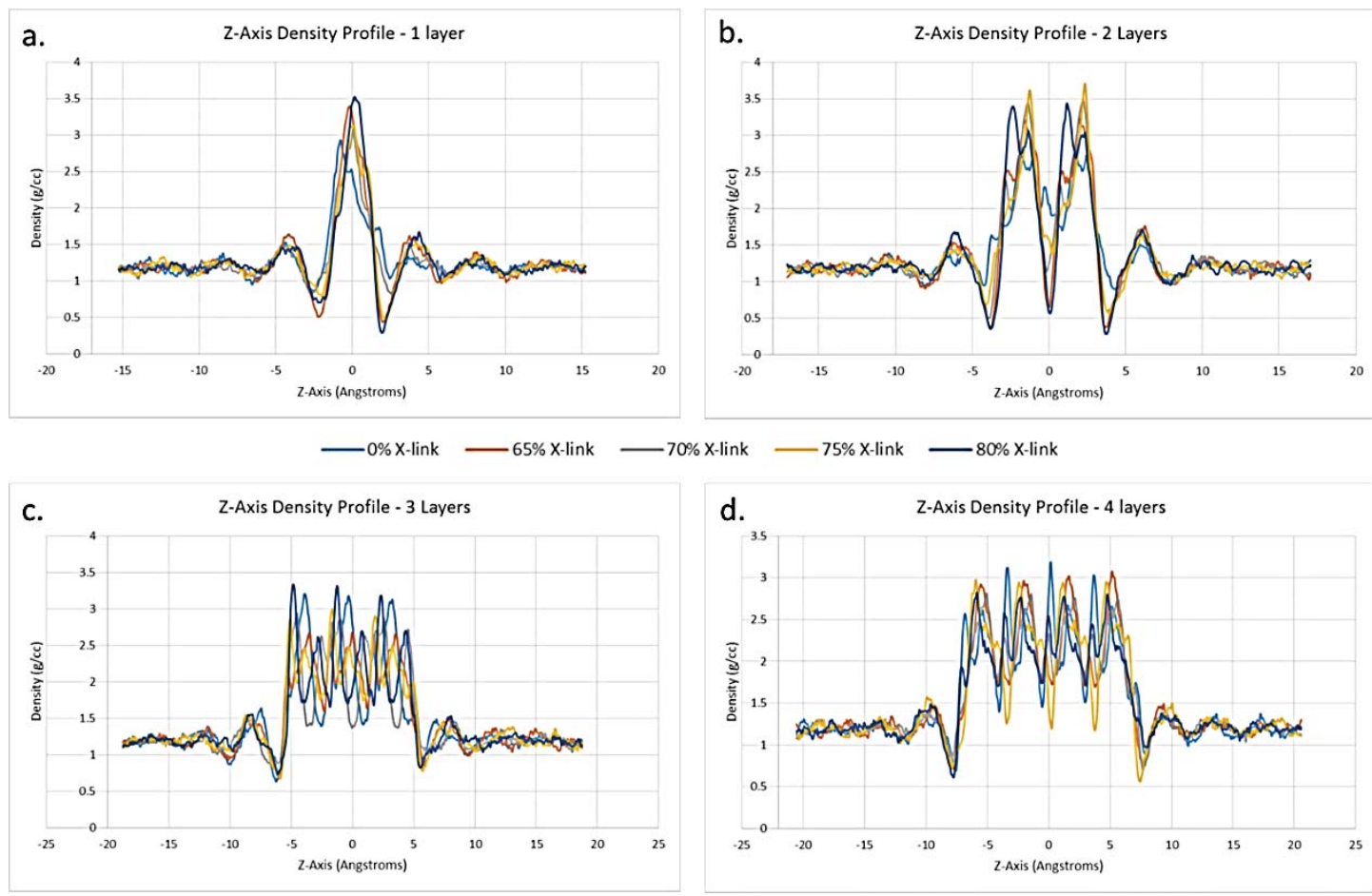

Figure 4.8 Atom density profile along z-axis for a)1 layer b) 2 layers c) 3 layers and d) 4 layers of graphene

Mechanical deformation results for each system can be seen in Table 4.2. Young's modulus increases with increasing layers of graphene but shows little influence from cross-link density. Deformation simulations in the $x-y$ plane produced stress vs. strain curves with very little scatter due to the fact that most of the force 
was placed upon the graphene structures. A visual representation of this can be seen in Figure 4.9, showing atomic stress values for tensile deformation parallel to the graphene surface. Shear values for $\mathrm{x}-\mathrm{z}$ and $\mathrm{y}-\mathrm{z}$ plane deformation along with Poisson's ratios for Z-axis deformation show a lot of scatter because van der waals attraction between polymer and graphene atoms allows them to easily slide past one another. Therefore, shear values for Gxz $=$ Gyz were very close to zero and were given a value of $1 \mathrm{MPa}$ for all models. In order to say that the models were truly transversely isotropic (i.e. Exx = Eyy, Vxy = Vyx, etc.), which requires less input for the MAC/GMC software, all applicable isotropic values were taken to be the average between the two.

Table 4.2 Resulting data from MD simulations

\begin{tabular}{|c|c|c|c|c|c|c|c|c|c|c|c|c|}
\hline MD Models & Polymer $\rho$ (g/cc) & $V_{f}$ graphene & Exx (Gpa) & Eyy (Gpa) & Ezz (Gpa) & Gxy (Gpa) & Vxy & Vyx & Vxz & Vyz & $\mathbf{V z x}$ & Vzy \\
\hline $65 \% 1$ layer & 1.124 & 0.111 & 94.035 & 94.235 & 2.397 & 0.242 & 0.132 & 0.148 & 0.636 & 0.59 & 0.0178 & 0.004 \\
\hline $65 \% 2$ layers & 1.127 & 0.187 & 177.434 & 175.749 & 2.846 & 0.433 & 0.153 & 0.152 & 0.615 & 0.584 & 0.012 & 0.071 \\
\hline 65\% 3 layers & 1.127 & 0.271 & 240.457 & 238.053 & 2.855 & 0.58 & 0.155 & 0.15 & 0.49 & 0.479 & 0.002 & 0.033 \\
\hline 65\% 4 layers & 1.126 & 0.33 & 294.361 & 291.922 & 3.218 & 0.705 & 0.153 & 0.153 & 0.489 & 0.501 & 0.007 & 0.073 \\
\hline $70 \% 1$ layer & 1.127 & 0.111 & 93.288 & 93.006 & 2.59 & 0.29 & 0.142 & 0.146 & 0.491 & 0.5 & 0.042 & 0.083 \\
\hline $70 \% 2$ layers & 129 & 0.187 & 170.408 & 170.695 & 3.008 & 0.424 & 0.146 & 0.159 & 0.468 & 0.462 & 0.042 & 0.007 \\
\hline $70 \% 3$ layers & 1.131 & 0.271 & 239.95 & 236.773 & 2.815 & 0.483 & 0.153 & 0.151 & 0.5 & 0.49 & 0.01 & 0.023 \\
\hline $70 \% 4$ layers & 13 & 33 & 294.502 & 295.313 & 3.294 & 0.542 & 0.153 & 0.155 & 0.507 & 0.454 & 0.011 & 0.035 \\
\hline $75 \% 1$ layer & 1.121 & 0.111 & 91.939 & 93.826 & 2.684 & 0.234 & 0.144 & 0.157 & 0.516 & 0.564 & 0.095 & 0.049 \\
\hline $75 \% 2$ layers & 13 & 0.187 & 174.797 & 175.239 & 2.768 & 0.429 & 0.154 & 0.163 & 0.55 & 0.516 & 0.0173 & 0.018 \\
\hline $75 \% 3$ layers & 1.129 & 0.271 & 238.646 & 238.057 & 3.034 & 0.579 & 0.154 & 0.151 & 0.514 & 0.493 & 0.02 & 0.004 \\
\hline $75 \% 4$ layers & 1.131 & 0.33 & 293.501 & 293.373 & 3.244 & 0.713 & 0.163 & 0.154 & 0.483 & 0.535 & 0.007 & 0.01 \\
\hline $80 \% 1$ layer & 1.133 & 0.111 & 93.377 & 94.826 & 2.432 & 0.243 & 0.13 & 0.158 & 0.46 & 0.471 & 0.109 & 0.025 \\
\hline $80 \% 2$ layers & 1.135 & 0.187 & 174.638 & 172.456 & 2.731 & 0.424 & 0.168 & 0.153 & 0.437 & 0.524 & 0.056 & 0.015 \\
\hline $80 \% 3$ layers & 1.132 & 0.271 & 239.449 & 238.336 & 3.005 & 0.582 & 0.152 & 0.151 & 0.44 & 0.446 & 0.01 & 0.034 \\
\hline $80 \% 4$ layers & 1.135 & 0.33 & 293.106 & 295.502 & 3.251 & 0.725 & 0.159 & 0.156 & 0.455 & 0.452 & 0.011 & 0.009 \\
\hline
\end{tabular}

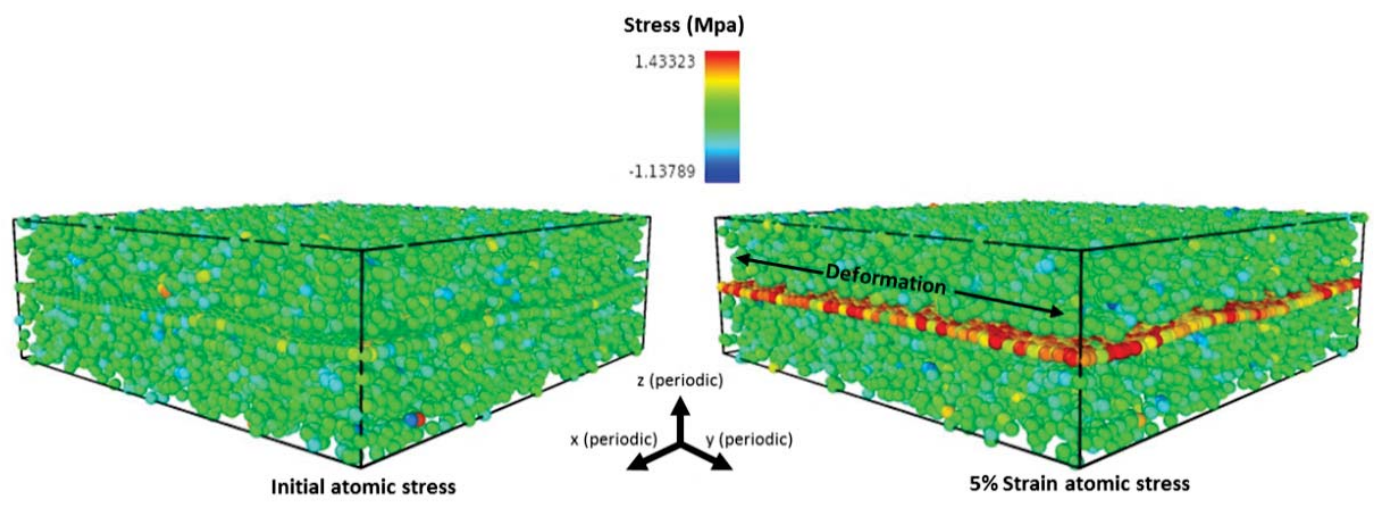

Figure 4.9 Atomic stress before and after tensile deformation along the y-axis. 


\section{Micromechanics using MAC/GMC software}

The mechanical properties derived from MD were implanted into the MACGMC software as a representation of a GNP with varying layers surrounded by interfacial polymer atoms. The amount of GNP that would contribute to the micromechanics calculations was controlled by volume fraction of graphene. Again, this calculation would incorporate the graphene volume fractions from MD for varying number of layers. The elastic modulus for bulk EPON 862 at room temperature was taken to be $2.72 \mathrm{GPa}^{10}$. Using the micromechanics software, representations of GNP doped EPON 862 were created with up to 5\% volume fraction of GNPs for varying number of graphene layers and crosslink densities. Results are shown in Figure 4.10, and indicate that GNPs with fewer layers will have a greater effect on the mechanical properties of this particular system. The single layer graphene systems were also the only systems to show any influence from increasing number of crosslinks (curing), likely because the MACGMC calculations for GNP volume fraction would need to incorporate more contributions from MD models for a single layer of graphene when compared to MD contributions for multi-layer models. Because crosslink density had little effect on the macroscopic properties predicted by MAC/GMC, only the data for

$80 \%$ crosslinked structures would be considered for the construction of the GNP doped epoxy/carbon fiber micromechanics models described later. 


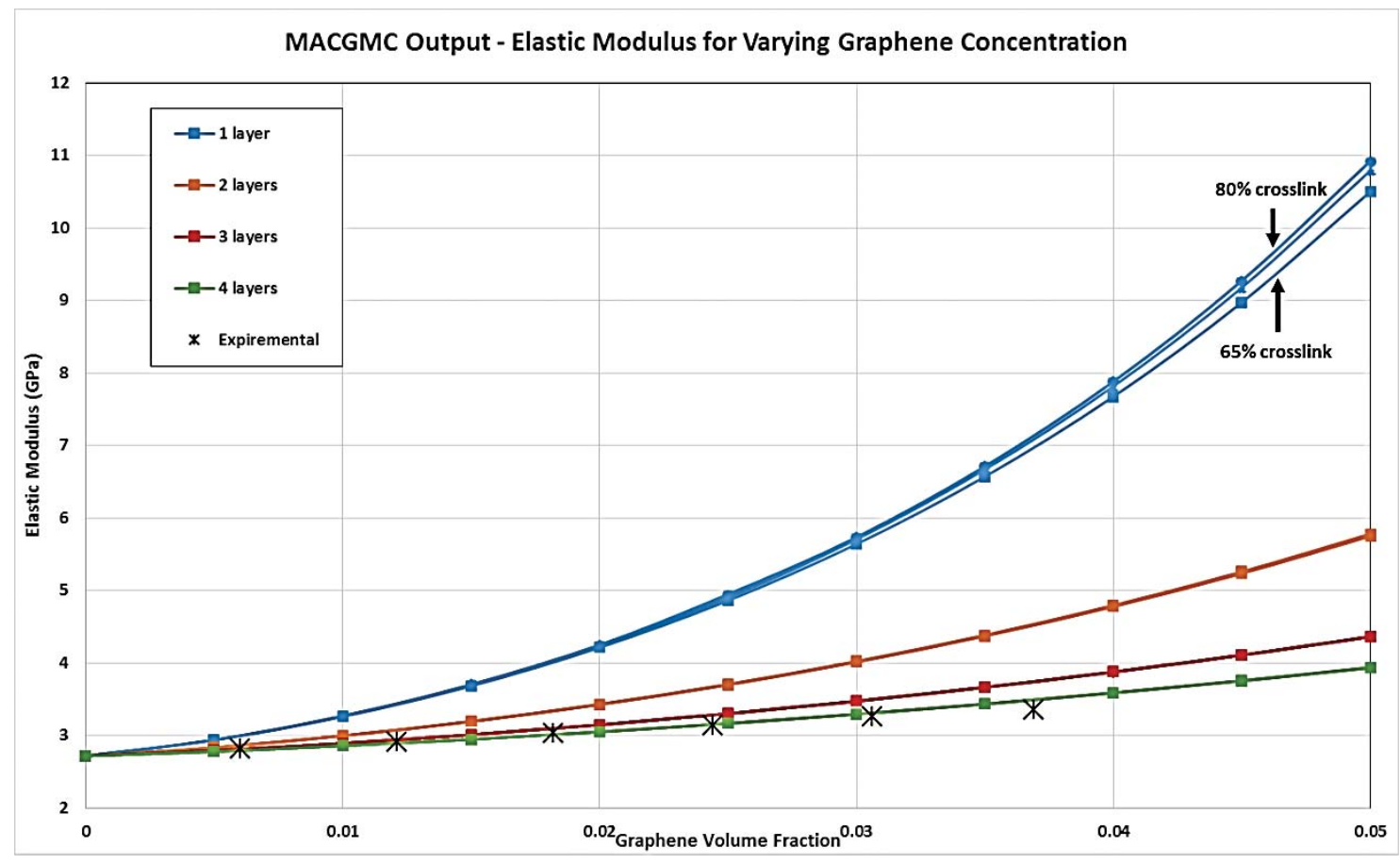

Figure 4.10 MAC/GMC data for addition of GNPs with carrying number of layers and interfacial polymer crosslink density.

The experimental data used for comparison was taken from Dr. J. King et al. ${ }^{10}$ where tensile test specimens were constructed with EPON 862 / DETDA epoxy and $\mathrm{xGnP}^{\circledR}{ }_{-} \mathrm{C}-300 \mathrm{GNPs}$ from XG sciences. ${ }^{54}$ The average GNP thickness was $2 \mathrm{~nm}$ before mixing, which would equate to 7-9 layers of graphene. The mixing process may also further break down the GNPs and reduce the overall particle thickness. Experimental results agree well with the data generated from the MD models with 3 or more layers of graphene, as seen in Figure 4.10. Results for the 1 and 2 layer GNPs produce significant increases in Elastic Modulus, though fabrication techniques for producing GNPs with a thicknesses less than $1 \mathrm{~nm}$ have not yet been developed.

Results for the MAC/GMC models considering GNP doped epoxy/carbon fiber composites are shown in Figure 4.11, and show a slight increase in modulus values for increasing GNP volume fractions. As expected, results for GNPs of a single atomic layer produce the greatest increases in modulus values. Experimental results for comparison were taken from Dr. King et al. for test specimens constructed of HexTow ${ }^{\circledR}$ AS4-GP/3K carbon fibers with the same $\mathrm{xGnP}^{\circledR}-\mathrm{C}-300$ GNPs used in the 
epoxy/GNP specimens previously mentioned. Experimental results are shown in Table 4.3, and indicate a decrease in tensile modulus with increasing GNP volume fractions. Though the model data and the experimental data observe different trends, the model data falls within the standard deviation of the experimental results for all GNP volume fractions, as shown in Figure 4.12. Transverse modulus results from MAC/GMC observe a similar trend as tensile modulus, increasing with higher GNP volume fractions and the largest increases are seen in the single layer model. Transverse modulus values are shown in Figure 4.13.

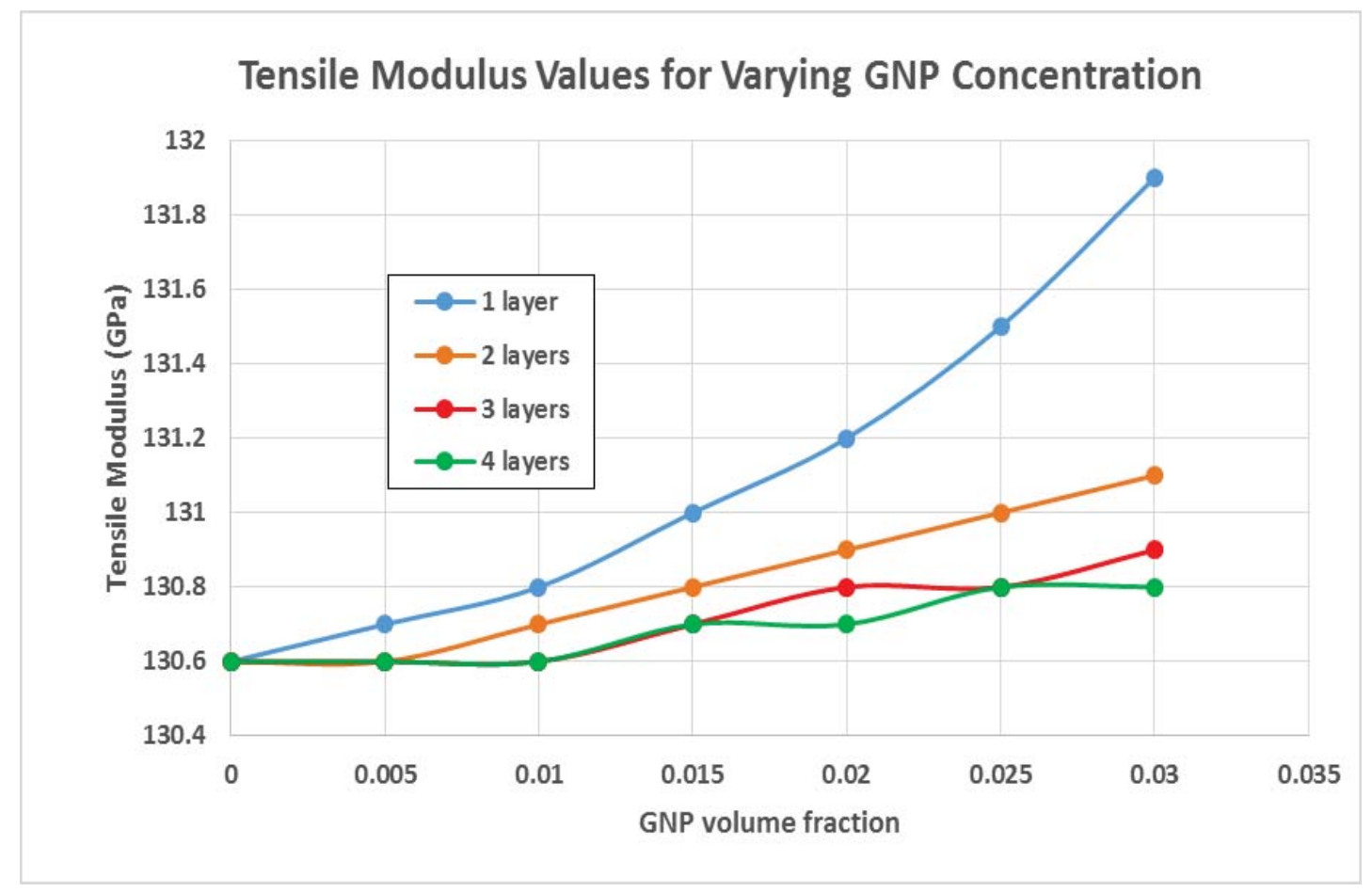

Figure 4.11 MAC/GMC tensile modulus for GNP doped epoxy/carbon fiber models for varying GNP volume fractions and atomic thicknesses. 
Table 4.3 Experimental results for epoxy/carbon fiber composites with varying GNP volume fractions

\begin{tabular}{cccc}
$\begin{array}{c}\text { GNP volume } \\
\text { fraction }\end{array}$ & Sample Size & $\begin{array}{c}\text { Tensile Modulus } \\
(\mathrm{GPa})\end{array}$ & $\begin{array}{c}\text { Standard } \\
\text { Deviation }\end{array}$ \\
\hline 0 & 8 & 158.9 & 9.09 \\
0 & 5 & 157.5 & 33.84 \\
0 & 6 & 136.6 & 25.33 \\
\hline 0.008 & 6 & 155.6 & 62.13 \\
0.008 & 7 & 137.6 & 10.92 \\
0.008 & 7 & 149 & 21.01 \\
\hline 0.016 & 6 & 152.6 & 15.03 \\
0.016 & 7 & 133.4 & 17.84 \\
0.016 & 6 & 129.1 & 13.35 \\
\hline 0.023 & 6 & 121 & 16 \\
0.023 & 6 & 128 & 6.27 \\
0.023 & 5 & 133 & 8.19 \\
\hline
\end{tabular}

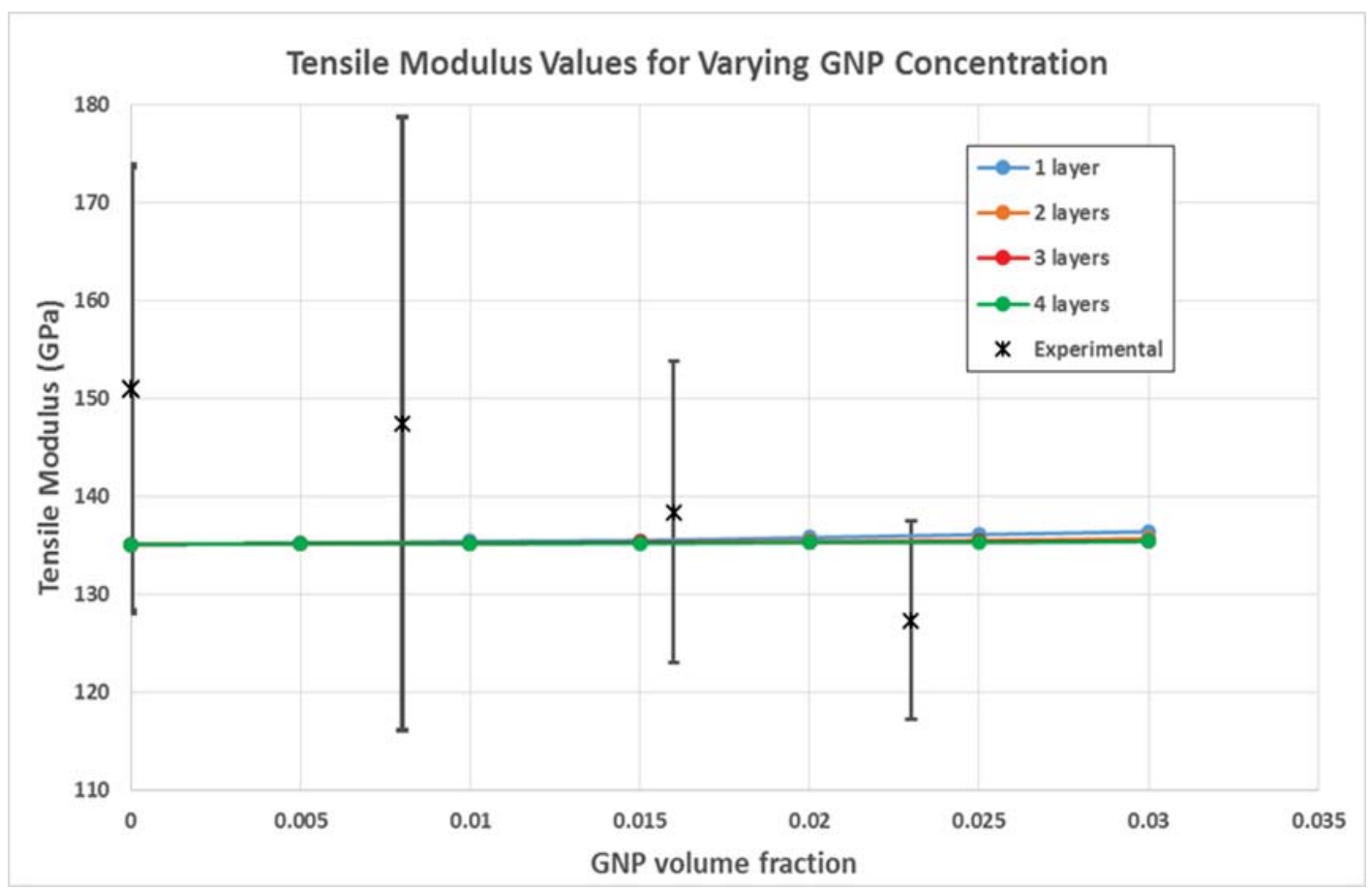

Figure 4.12 Experimental and MAC/GMC tensile modulus for GNP doped epoxy/carbon fiber models for varying GNP volume fractions and atomic thicknesses. 


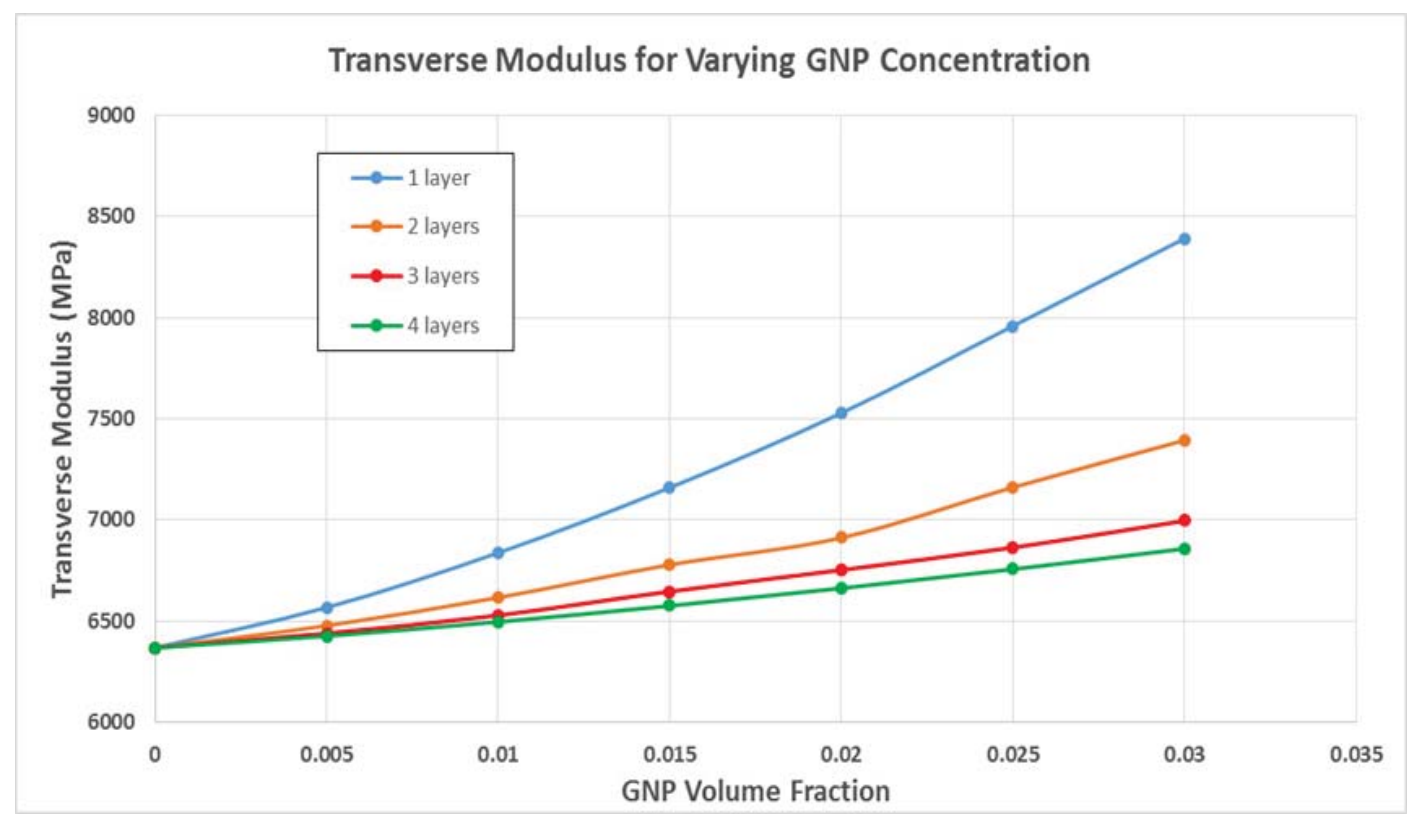

Figure 4.13 MAC/GMC transverse modulus for GNP doped epoxy/carbon fiber models for varying GNP volume fractions and atomic thicknesses.

\subsection{Conclusions}

In this study, MD techniques were utilized to determine the influence of crosslink density and GNP atomic thickness on molecular level mechanical properties. The models were designed to focus on the interfacial polymer surrounding an embedded graphene structure with different numbers of atomic layers. Models show an interfacial region of $\sim 10 \AA$ in which the mass density profile behaves differently than the bulk polymer, further validating previous works. Mechanical properties derived from MD models such as tensile modulus and shear modulus increase with increasing number of graphene atomic layers. Varying crosslink density shows no significant effect on mechanical properties for the range investigated by MD models. Micromechanics models were created for GNP doped epoxy specimens using MAC/GMC software, with inputs from MD results. The micromechanics analysis was designed to create a fully isotropic representation of a GNP doped epoxy 
specimen with GNPs of varying atomic thickness and GNP volume fraction. Results show increases in Elastic Modulus for increasing GNP volume fractions, with the greatest increases in modulus coming from GNPs of a single atomic layer and decreasing as more layers are considered. Results from MAC/GMC for GNP doped epoxy specimens are in good agreement with experimental data, especially for modelled GNPs of 3 or more atomic layers. MAC/GMC models were next created for GNP doped epoxy/carbon fiber composites. These composite models show slight increases in both tensile and transverse modulus values with increasing GNP volume fractions in the surrounding matrix epoxy. Again, GNPs of a single atomic layer produced the most significant increase. Experimental comparison shows the composite models to fall within the Standard Deviation for all sampled GNP volume fractions and further validate this method for utilizing MD data to develop larger scale models of composite materials.

\section{CHAPTER 5: MULTI-SCALE MODELING OF GRAPHENE OXIDE / EPOXY COMPOSITES ${ }^{2}$}

The fabrication of carbon fibers and graphene nanoplatelets (GNPs) will often leave oxygen species bonded to the surface of the carbon structures. In this chapter, different oxygen species are attached in different amounts to a single layer graphene surface and a 4 layer graphene surface. The results are compared to previous data for pristine graphite structures.

\subsection{Introduction}

The surface of graphite structures often contains small amounts of oxygen and even smaller amounts of nitrogen that are deposited during the fabrication process. ${ }^{55}$ These groups can influence the behavior of the interfacial region between

\footnotetext{
${ }^{2}$ The information from this chapter will be considered for publication in the near future.
} 
the surface of the graphite structure and the surrounding epoxy matrix. There are many accepted models for the structure of graphene oxide, as shown in Figure 5.1. ${ }^{56}$ Experimental techniques such as X-ray photoelectron spectroscopy (XPS) can help reveal the exact oxygen species present, along with a rough estimate of their atomic composition for the tested graphite structure. ${ }^{57}$ The use of molecular dynamics (MD), coupled with knowledge gained from experimental data, can be a powerful tool in discovering the effects oxygen on the surface of a carbon structure. MD is able to provide insight into: the effects of adding specific oxygen species, the effects of varying the amount of these species on the graphite surface, the likely pattern or confirmation of which these oxygen species will attach to the surface of a graphite structure. All of which are still topics of relative uncertainty and or unpredictability.
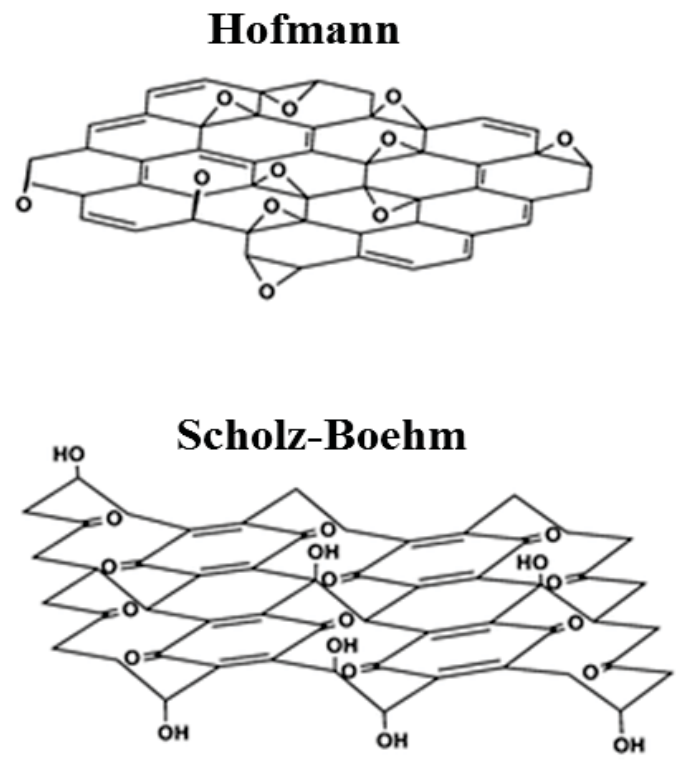

\section{Ruess}
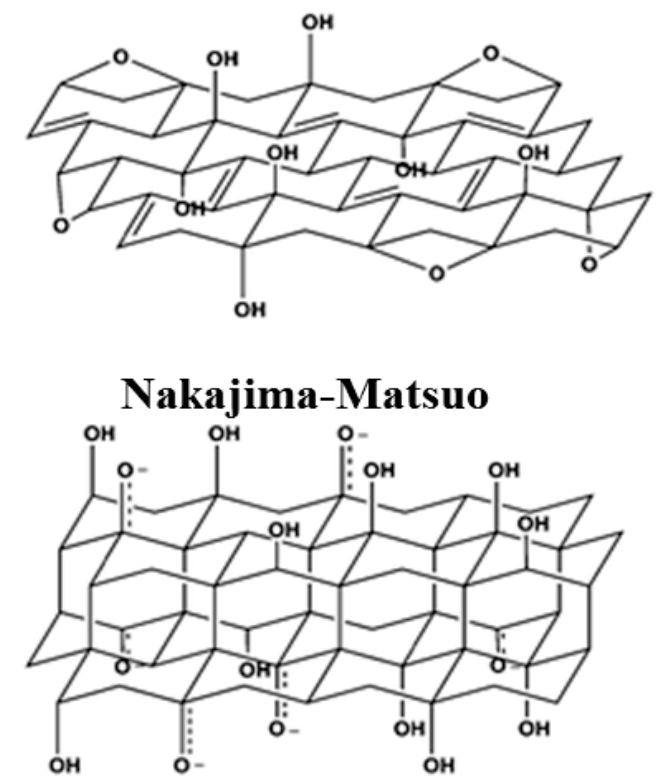

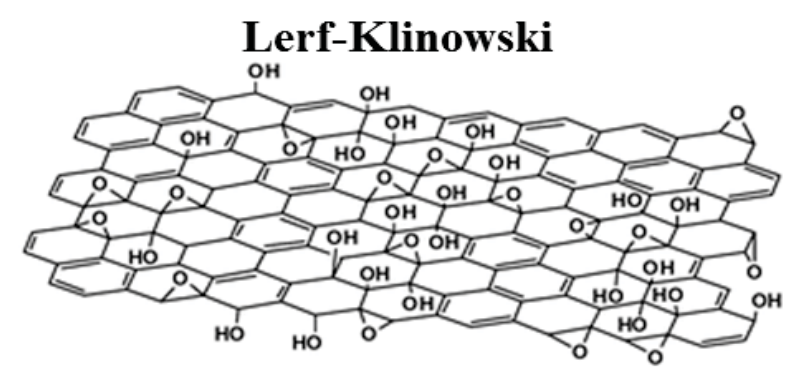

Figure 5.1 Widely accepted models for the structure of graphene oxide. 
The use of MD in relation to graphite oxide has been explored by several researchers. ${ }^{58-64}$ Early models by Allington et. al. incorporate regularly spaced oxygen species $(\mathrm{OH}, \mathrm{CO}, \mathrm{COOH})$ on a graphitic surface and report their interaction energies with small molecules ${ }^{58,59}$ I.S. Awan et. al. utilized MD to construct models of oxidized carbon fiber surfaces, varying concentrations of various oxygen species and reporting the changes is surface energy. These studies have provided valuable information regarding the molecular structure of oxidized graphite, though there has been little effort to investigate the effects on mechanical properties.

The purpose of this study was to create models of oxidized graphene surfaces surrounded on both sides by EPON 862/DETDA epoxy. The data provided from referenced MD research would become the basis for deciding which oxygen species to add, the concentration of oxygen species upon the graphite surface, and the bonding arrangement on the graphite surfaces. Models were created for graphene oxide with the addition of epoxide oxygen species, hydroxyl oxygen species, and a combination of epoxide and hydroxyl oxygen species. These three models were created for graphene structures of 1 and 4 atomic layers. The amount of oxygen present on the surface was also varied for amounts of $12 \%$ and $24 \%$ oxygen. The influences of these 3 variables are investigated within the MD models. Mechanical

properties are then derived from the MD models and compared to results for pristine graphene models from Chapter 4. Results show oxygen groups to cause a decline in modulus values in all cases. The addition of epoxide oxygen species show the least amount of reduction when compared to pristine graphene structures.

\subsection{Modeling Procedures}

The molecular models described in this chapter were created nearly exactly the same as those from Chapter 4. The initial 2:1 Stoichiometric ratio of EPON 862:DETDA was replicated into a 250:125 ratio, and this structure was mirrored 
about a central graphene oxide structure. Therefore, the final ration of EPON:DETDA was 500:250 totaling 20,750 polymer atoms. Each layer of graphene was again composed of 4200 carbon atoms, and the box size was kept the same. The focus of this chapter was the effect of oxidizing the graphene surface. In order to save time, graphene structures were created composed of only a single atomic layer and of 4 atomic layers. This would enable comparison to the optimal result from the previous chapter (single layer graphene) and the most realistic result from the previous chapter (4 layer graphene). The oxygen species chosen for this project were based on a study performed by D. W. Boukhvalov et. al., in which Density Functional Theory (DFT) was used in conjunction with the widely accepted models of graphite oxide (Figure 5.1) to identify the most stable attachment of oxygen species to graphite structures. ${ }^{61}$ From this research, The three most stable configurations for the attachment of oxygen species to a graphite surface were chosen to be implemented into the MD model. They can be seen in Figure 5.2. Models were created with epoxide molecules only (Fig. 5.2a), hydroxyl molecules only (Fig 5.2b), and a combination of epoxide and hydroxyl molecules (Figure 5.2c). The lowest energy configuration, as found by DFT, was the epoxide only model. The next lowest energy was that for the hydroxyl only model and the highest energy confirmation was the combination model. ${ }^{61}$ Each of these oxygen species would be attached to the single sheet models, along with the outer most layer of the 4 sheet models, in a regular pattern in varying amounts. A total of 12 models were created varying in: number of graphene layers ( 1 and 4 layered structures), the attached oxygen species (epoxide, hydroxyl, and a combination), and the amount of each oxygen species. The amounts of oxygen are described in this research as a percentage, and correspond to the number of carbon atoms that they are attached to. If oxygen species were attached to 420 of the 4200 carbon atoms, that would equate to $10 \%$. The amounts chosen for this work were $12 \%$ and $24 \%$, which are in good agreement with referenced data. ${ }^{55-64}$ Figure 5.3 shows the fully equilibrated graphene oxide structures of both the single and 4 atomic layers for the 3 possible combinations covering $12 \%$ of the carbon atoms. As shown in Figure 5.2, oxygen 
species protrude from both sides of the graphene surface. This is difficult to see from Figure 5.3, but the focus of the figure was the regular pattern in which oxygen species were bonded.

a)

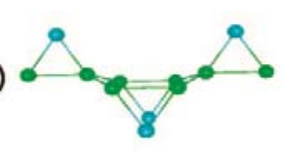

b)
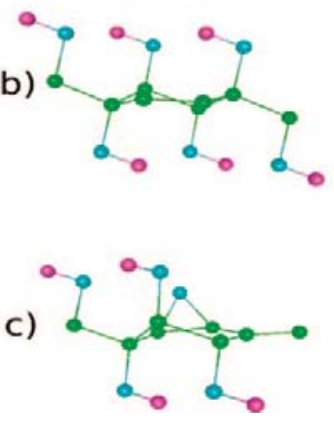
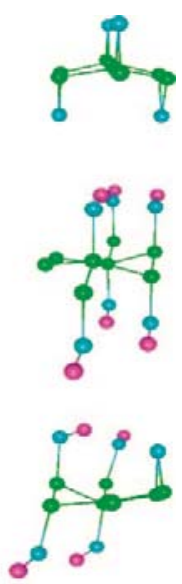

Figure 5.2 The most stable confirmations of graphene oxide as found by DFT for a) epoxide oxygen only, b) hydroxyl molecules only, and $\mathbf{c}$ ) a combination of epoxide and hydroxyl molecules. Carbon molecules are shown to be green, oxygen is blue, and hydrogen is pink.
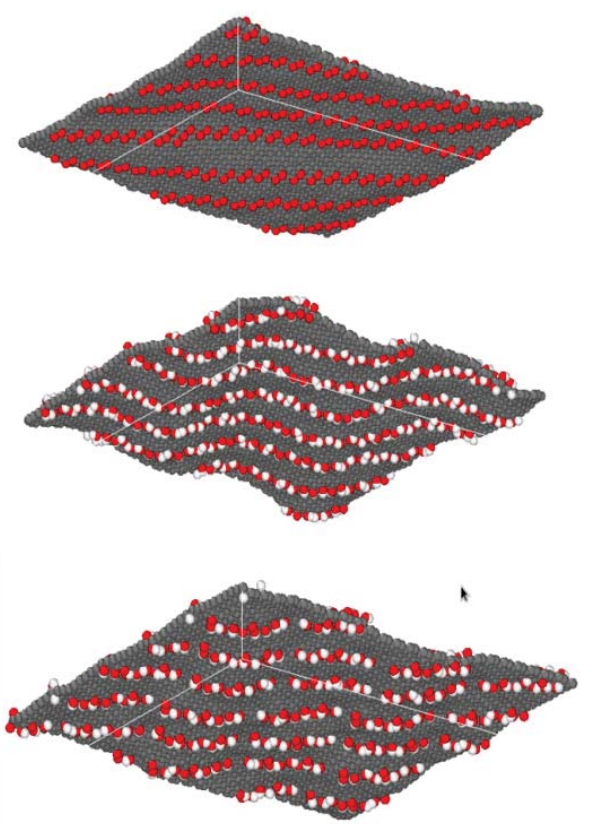

Hydroxyl

Epoxide

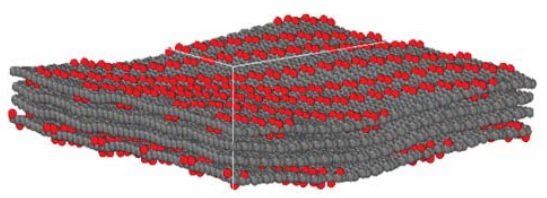

Combo
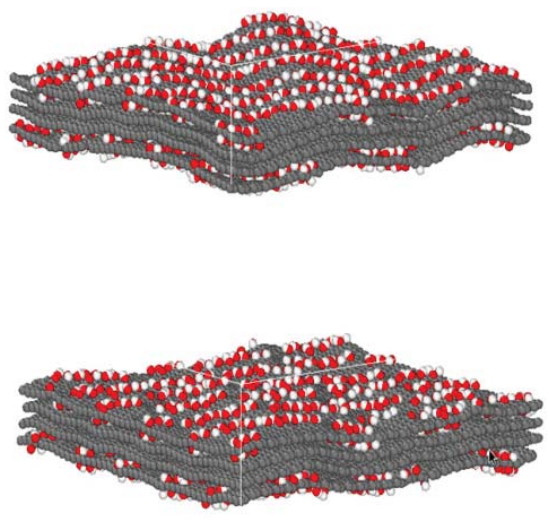

Figure 5.3 Single and 4 layer graphene oxide structures containing 12\% bonded oxygen species. Carbon atoms are gray, oxygen is red, and hydrogen is white. Oxygen atoms protrude from the top and bottom of the single layer, and the outermost layers of the 4 layer structures. 
After creating the models of graphene oxide and adding the polymer atoms as described above. The models were densified in the same manner as in Chapter 4. All models were condensed to very near the target density, and were then crosslinked. The crosslinking procedure was the same as in previous works and is described in detail in section 2.3 (page 12). All models were crosslinked to 75\% crosslink density, as results from the previous study for pristine graphite revealed little effect for varying the amount of crosslinks. Fully equilibrated crosslinked structures for 1 and 4 layer graphene with the 3 different oxygen species are shown in Figure 5.4 for $12 \%$ bonded oxygen. Before deforming the equilibrated models, the pressure was relived using an NPT simulation causing an increase in the simulation box size for all 3 dimensions. In order to keep the density near the target, a final MD densification was performed along the z-axis. Density values for each model can be seen in Table 5.1
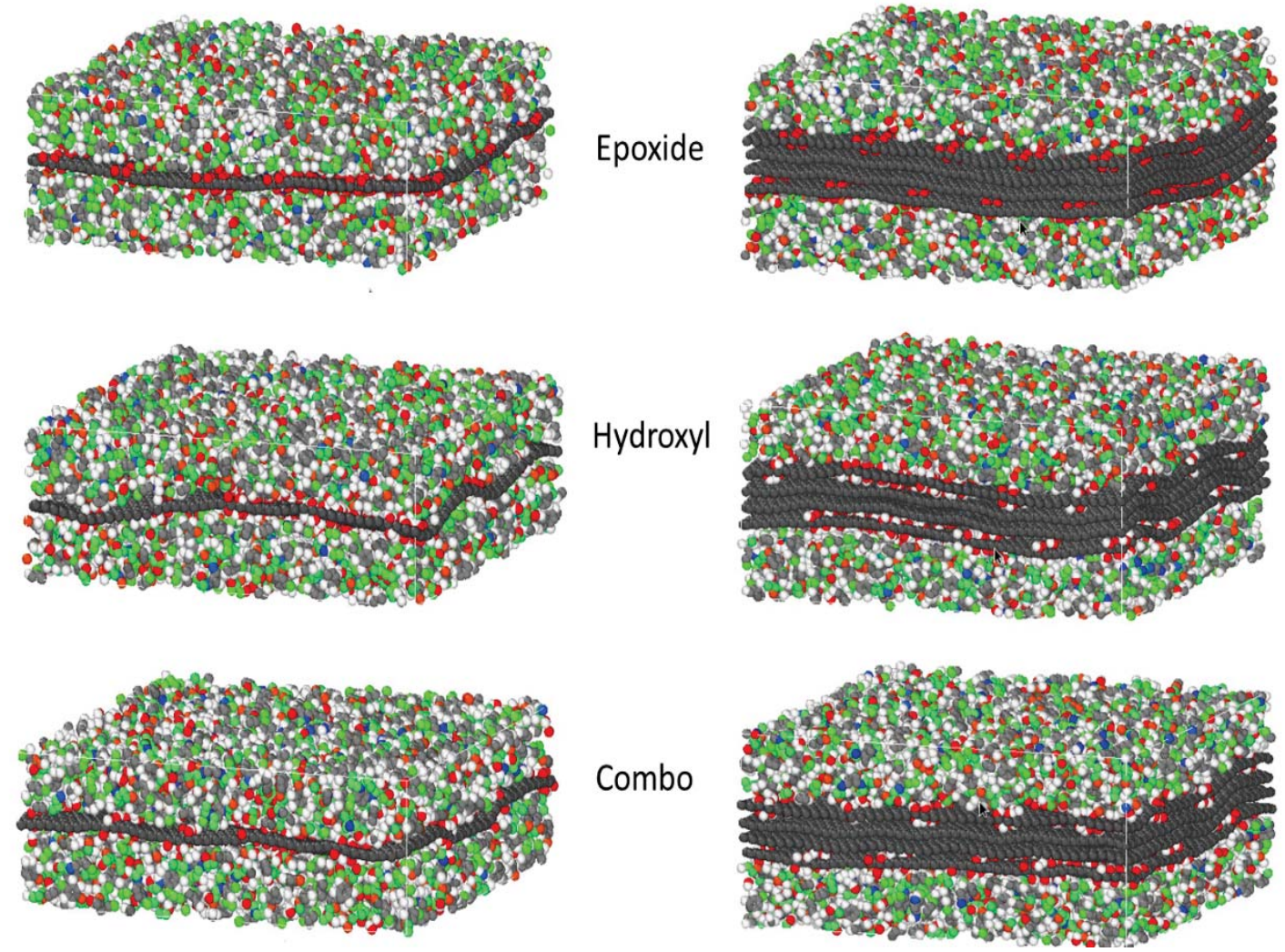

Figure 5.4 Fully equilibrated Single and 4 layer graphene oxide / epoxy structures containing $12 \%$ bonded oxygen species. 
Deformation simulations were then performed in both Tension and Shear, in the exact manner as the previous chapter. Models were deformed to 5\% strain, providing tensile and shear modulus data, as well as Poisson's ratio. Exact details are provided in section 4.2 (page 34). All mechanical properties derived from the MD models can be seen in Table 5.1 below. After deforming, results from the epoxide only models were implemented into MAC/GMC software and compared to the results for $75 \%$ crosslinked pristine GNP structures of 1 and 4 atomic thicknesses.

\subsection{Results and Discussion}

The interfacial polymer mass density profiles for the oxidized graphene show less fluctuation near the graphene surface, as shown in Figure 5.5. This is likely due to the fact that atoms are able to protrude between the strips of attached oxygen species, making them appear more even. When examining the oxidized graphene structures in Figure 5.2, each attached oxygen species causes a different amount of distortion to the graphene surface. The amount of distortion (waviness) seen in each of the models is related to the energy of the oxidized structure. As would be expected, the epoxide only models show the least amount of distortion and the peaks in mass density profile are easily visible for each layer. The hydroxyl models are very wavy, causing the layers to be less distinguishable in the figure. This wavy behavior is synonymous with buckling and proved to be a problem during deformation, highlighted later. 

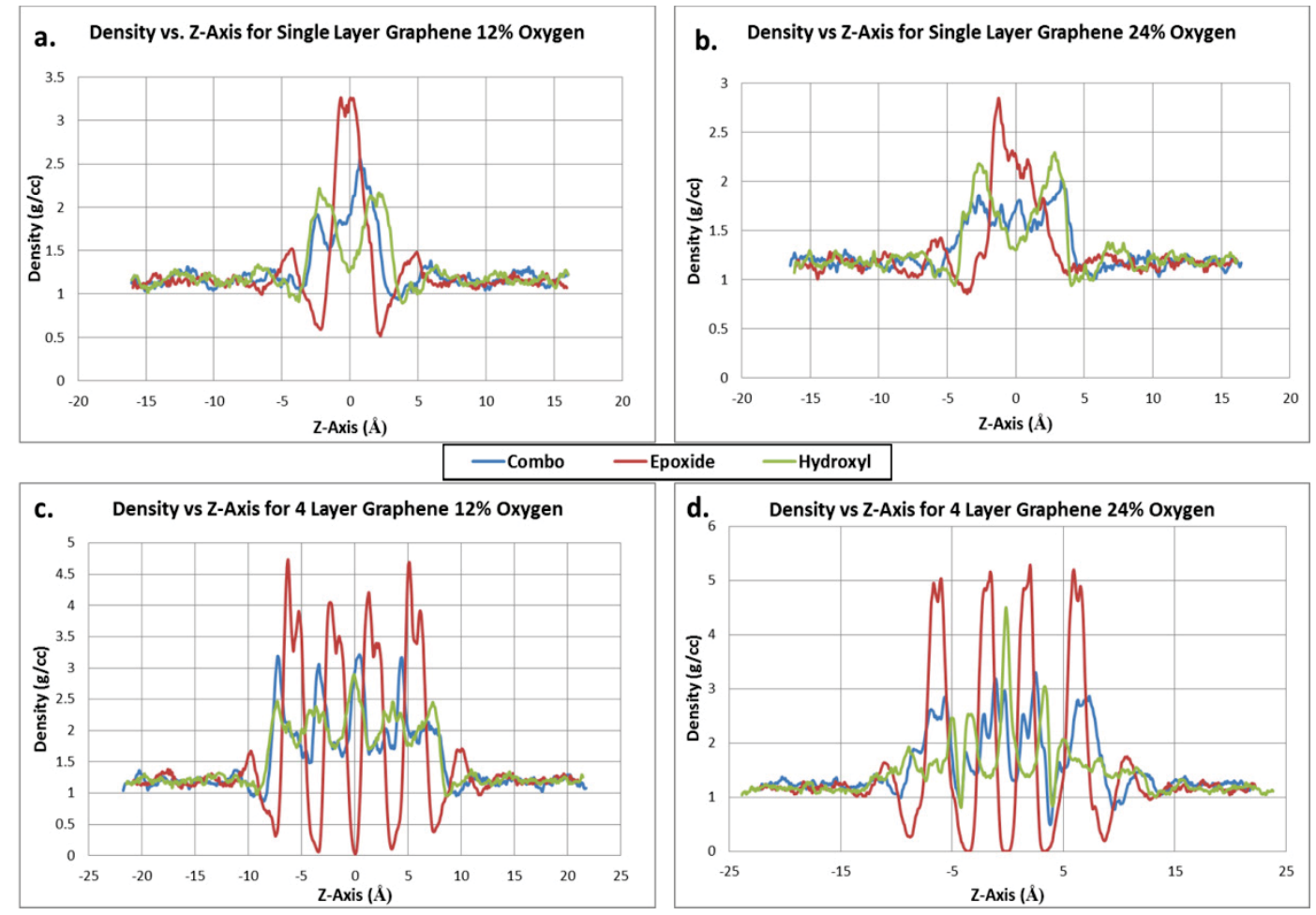

Figure 5.5 Polymer mass density profile viewed along the Z-Axis for single layer a) $12 \%$ bonded oxygen b) $24 \%$ bonded oxygen and 4 layer c) $12 \%$ bonded oxygen d) $24 \%$ bonded oxygen

Upon noticing the wavy behavior is the hydroxyl and combination models, the per atom potential energy values were investigated. Figure 5.6 shows the energy values for each atom within the graphene oxide structures for single and 4 layer graphene oxide structures with varying oxygen species at $12 \%$ oxygen bonding. The epoxide atoms seem to produce the highest energy values. This seems to go against the referenced values from DFT, though those were calculated for the graphene oxide as a whole rather than just the epoxide groups alone. In terms of bond energy, the epoxide bond is a high energy bond, which is why it is a common reaction site. Hydroxyl atoms cause the graphene to undulate, but are at a relatively low energy state. The highest energy contributions from the combination graphene oxide models are due to the epoxide molecules. The high energy bonding of the epoxide oxygen molecules appears to contribute to the more planar structure. The undulated behavior seen from the hydroxyl molecules results from the changing of the Carbon to Carbon bond distances in the aromatic rings to which these molecules are bonded. 

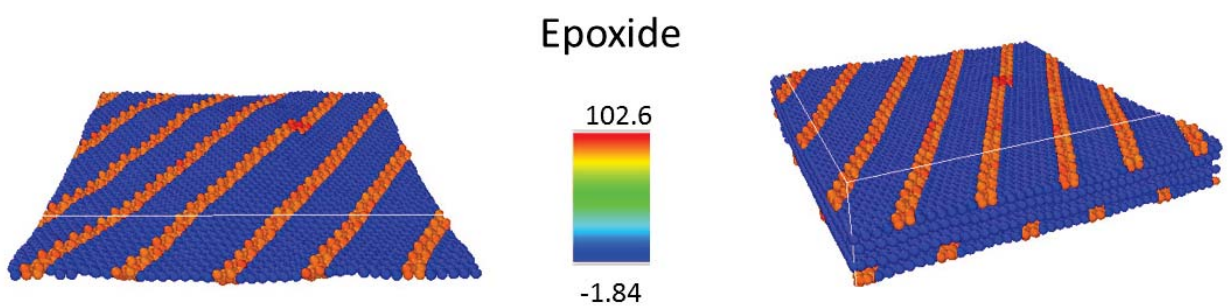

\section{Hydroxyl}
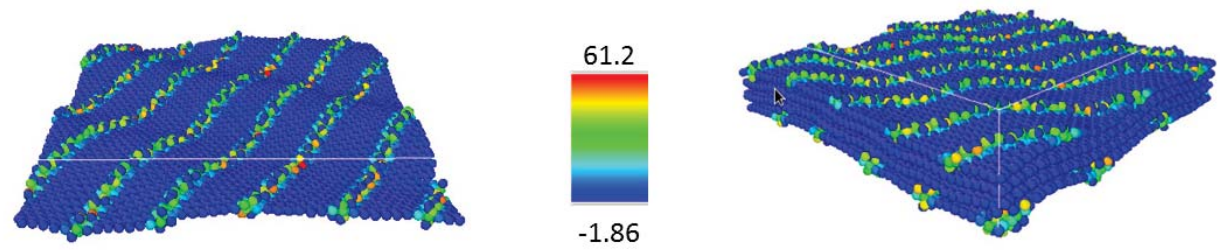

Combo
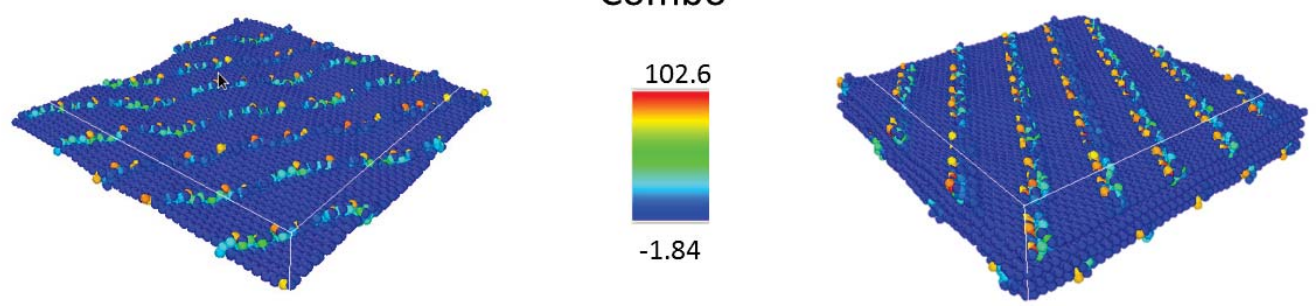

Figure 5.6 Visual representation of per-atom potential energy values (Kcal/mol-Angstrom) for single and 4 layer graphite oxide structures for varying oxygen species with $12 \%$ oxygen bonding.

Mechanical properties are derived from stress vs. strain curves that look very similar to those shown in Figure 4.5 from Chapter 4. All mechanical properties for oxidized graphene models are shown in Table 5.1. Data from the 75\% crosslinked pristine graphene structures from Chapter 4 are also shown for comparison. When allowing for Poisson's contractions during tensile deformation, the wavy hydroxyl group containing structures extended outward rather than contracting. This occurs even after relieving the pressure along the $\mathrm{x}$ and $\mathrm{y}$ axes with a 2 nanosecond NPT simulation. The Poisson's values for which this phenomenon occurred simply have a (+) value in the table, as the deformation of the oxidized graphene sheets in the axial direction was coupled by extension transverse direction. This may be attributed to the pattern in which the oxygen species were attached to the graphene surface. The addition of oxygen species reduces the $\mathrm{x}$ and $\mathrm{y}$ axis (axial) tensile modulus values when compared to that for pristine graphene structures. This effect is amplified by 
adding more oxygen. The $\mathrm{z}$ axis (transverse) modulus values show an increase for the oxygenated graphene structures when compared to pristine graphene, likely due to stronger atomic interactions between the attached oxygen species and the surrounding polymer atoms. Results for shear modulus along the xy plane are decreased for the hydroxyl and combination models, though the epoxide models produce slightly better results than that of pristine graphite. Unlike the results for pristine graphite, shear in the $\mathrm{xz}$ direction provided positive values for all of the models. The attached oxygen groups prevented the polymer atoms from easily sliding past the graphite surface. The models utilizing only epoxide oxygen species produced the best results for mechanical properties, including good results for Poisson's contractions and transverse isotropy, which would enable them to be implemented into the MAC/GMC software.

Table 5.1 Mechanical properties derived from MD for oxidized graphene models along with those for $75 \%$ crosslinked pristine graphene MD models from chapter 4 for comparison

\begin{tabular}{|c|c|c|c|c|c|c|c|c|c|c|c|c|}
\hline MD Models & Polymer p(g/cc) & $\operatorname{Exx}(\mathrm{Gpa})$ & $\mathrm{Eyy}(\mathrm{Gpa})$ & $\mathrm{Ezz}(\mathrm{Gpa})$ & $\mathrm{Gxy}(\mathrm{Gpa})$ & $\mathrm{Gxz}(\mathrm{Gpa})$ & $V_{x y}$ & $V_{y x}$ & $V_{x z}$ & $V_{y z}$ & $V_{z x}$ & $V_{z y}$ \\
\hline Pristine 1 Layer & 1.121 & 91.939 & 93.826 & 2.684 & $\mathbf{0 . 2 3 4}$ & $\mathbf{0 . 0 0 1}$ & $\mathbf{0 . 1 4 4}$ & $\mathbf{0 . 1 5 7}$ & $\mathbf{0 . 5 1 6}$ & $\mathbf{0 . 5 6 4}$ & $\mathbf{0 . 0 9 5}$ & $\mathbf{0 . 0 4 9}$ \\
\hline 12\% Combo 1 Layer & 1.16 & 78.6 & 68.7 & 5.29 & 0.173 & 0.021 & $(+)$ & 0.015 & 0.67 & 0.54 & 0.055 & 0.11 \\
\hline 12\% Epoxide 1 Layer & 1.15 & 92.1 & 93.1 & 4.67 & 0.244 & 0.013 & 0.133 & 0.13 & 0.48 & 0.37 & 0.042 & 0.041 \\
\hline 12\%Hydroxyl 1 Layer & 1.16 & 76.1 & 46.7 & 5.15 & 0.131 & 0.021 & $(+)$ & 0.018 & 0.75 & 0.53 & 0.064 & 0.11 \\
\hline 24\% Combo 1 Layer & 1.17 & 61.4 & 54.8 & 5.45 & 0.134 & 0.039 & $(+)$ & $(+)$ & 0.64 & 0.72 & 0.081 & 0.107 \\
\hline 24\% Epoxide 1 Layer & 1.16 & 85.5 & 91.7 & 5.44 & 0.29 & 0.023 & 0.102 & 0.14 & 0.48 & 0.47 & 0.068 & 0.034 \\
\hline 24\% Hydroxyl 1 Layer & 1.18 & 79.8 & 32.8 & 6.15 & 0.175 & 0.023 & $(+)$ & $(+)$ & 0.82 & 0.31 & 0.061 & 0.15 \\
\hline Pristine 4 Layers & 1.131 & 293.501 & 293.373 & 3.244 & 0.713 & 0.001 & 0.163 & 0.154 & 0.483 & 0.535 & 0.007 & 0.01 \\
\hline 12\% Combo 4 Layers & 1.17 & 238.3 & 239.7 & 8.07 & 0.477 & 0.003 & 0.086 & 0.104 & 0.39 & 0.53 & 0.045 & 0.051 \\
\hline 12\% Epoxide 4 Layers & 1.18 & 271.5 & 278.9 & 10.32 & 0.772 & 0.012 & 0.126 & 0.13 & 0.37 & 0.41 & 0.017 & 0.018 \\
\hline 12\% Hydroxyl 4 Layers & 1.19 & 227.3 & 201.9 & 8.97 & 0.38 & 0.015 & 0.074 & 0.109 & 0.49 & 0.72 & 0.038 & 0.082 \\
\hline 24\% Combo 4 Layers & 1.2 & 234.7 & 199.9 & 11.36 & 0.451 & 0.015 & 0.032 & 0.041 & 0.55 & 0.59 & 0.034 & 0.075 \\
\hline 24\% Epoxide 4 Layers & 1.15 & 264.1 & 262.6 & 7.4 & 0.742 & 0.005 & 0.136 & 0.13 & 0.364 & 0.49 & 0.016 & 0.018 \\
\hline 24\% Hydroxyl 4 Layers & 1.19 & 220.5 & 141.1 & 5.42 & 0.269 & 0.006 & 0.03 & 0.075 & 0.57 & 0.58 & 0.019 & 0.039 \\
\hline
\end{tabular}

MAC/GMC results for oxidized graphene composed of only epoxide species were very close to that for pristine graphite with $75 \%$ crosslinked epoxy, indicating little influence from increases in mechanical properties shown in Table 5.1.

MAC/GMC micromechanics results are shown for up to 5\% GNP concentration in Table 5.2. As seen from the table, pristine graphite structures produce best results. 
Table 5.2 MAC/GMC results for 75\% crosslinked pristine and epoxide oxidized graphite structures of 1 and 4 atomic layers.

\begin{tabular}{|c|c|c|c|c|c|c|}
\cline { 2 - 7 } & \multicolumn{3}{|c|}{ 1 Layer Graphene-Tensile Modulus(Gpa) } & \multicolumn{4}{|c|}{ 4 Layer Graphene-Tensile Modulus(Gpa) } \\
\hline Vf Graphene & Pristine & $12 \%$ Epoxide & 24\% Epoxide & Pristine & $12 \%$ Epoxide & $24 \%$ Epoxide \\
\hline 0 & 2.72 & 2.72 & 2.72 & 2.72 & 2.72 & 2.72 \\
\hline 0.005 & 2.941 & 2.938 & 2.938 & 2.781 & 2.781 & 2.781 \\
\hline 0.01 & 3.271 & 3.261 & 3.26 & 2.858 & 2.858 & 2.858 \\
\hline 0.015 & 3.704 & 3.684 & 3.68 & 2.949 & 2.948 & 2.948 \\
\hline 0.02 & 4.25 & 4.212 & 4.206 & 3.052 & 3.051 & 3.051 \\
\hline 0.025 & 4.92 & 4.858 & 4.848 & 3.167 & 3.166 & 3.166 \\
\hline 0.03 & 5.734 & 5.637 & 5.621 & 3.295 & 3.293 & 3.292 \\
\hline 0.035 & 6.712 & 6.567 & 6.542 & 3.435 & 3.433 & 3.432 \\
\hline 0.04 & 7.881 & 7.667 & 7.632 & 3.589 & 3.585 & 3.584 \\
\hline 0.045 & 9.271 & 8.964 & 8.914 & 3.755 & 3.751 & 3.75 \\
\hline 0.05 & 10.92 & 10.49 & 10.42 & 3.936 & 3.931 & 3.929 \\
\hline
\end{tabular}

\subsection{Conclusions}

This chapter investigates the addition of oxygen species to graphene to simulate graphene oxide in the presence of interfacial EPON 862-DETDA epoxy. Even after an extensive relaxation process, some models did not accurately reflect Poisson's contractions during tensile deformation. Perhaps attaching the oxygen in a different pattern would eliminate this problem. Interfacial characteristics agree with those for pristine graphene, though the oscillations in polymer mass density profiles are lessened due to the protruding hydroxyl molecules from the graphene surface. Per-atom potential energy values are highest in the epoxide molecules, due to the high energy of the epoxide bond. The addition of hydroxyl groups causes the graphene to become wavy, though this may be due to the pattern of which they were applied. Both elastic and shear modulus values are decreased with the addition of hydroxyl oxygen to the graphene structures, and more oxygen appears to amplify this effect. Epoxide only models are in best agreement with pristine graphene, followed by models with a combination of epoxide and hydroxyl molecules. Attaching only 
hydroxyl groups to the graphene surface produces the lowest values of tensile and shear modulus. The addition of oxygen species provides increases in values for $\mathrm{z}-$ axis tensile modulus and shear modulus in the $\mathrm{x}-\mathrm{z}$ plane, though these increases have little effect on the micromechanics results for a GNP doped composite.

\section{CHAPTER 6: SCOPE FOR FUTURE WORKS}

This chapter highlights the possibilities for future research in the realm of epoxy / carbon composites. Suggestions for improvements to the research in this report are also highlighted.

\subsection{Improving Modeling Procedures}

Throughout the research performed in this report, many possible changes to the modeling procedure were brought to attention. The area for the most improvement would be the densification equilibration and crosslinking procedures. Crosslinking in this work was performed after the material was brought very near to the target density of $1.17 \mathrm{~g} / \mathrm{cc}$. During the last research performed on the oxidized graphene, all the models were brought near to the target density before crosslinking. It was noticed that some models were able to achieve the target crosslink density of $75 \%$ much quicker than others. After investigating this, it was found that the models that were further away from the target density were able to crosslink much quicker. The reason for discrepancies in density from one model to another was that the oxygen groups attached to the graphene surfaces would produce different amounts of space between the graphene layers, along with the surrounding epoxy. More specifically, models were densified based strictly on the number of layers of graphene and the type of oxygen species attached to the surface was disregarded. 
This produced a different density in each model prior to crosslinking. When these materials crosslink, there is a small amount of volume shrinkage. Based on this, it could be proposed that models be brought very near the target and then crosslinked. The concept of volume shrinkage could be considered and employed during the densification/crosslinking procedure. With the LAMMPS software always improving, it is now possible to crosslink and densify at the same time all within the MD simulation. Performing these procedures statically outside of the actual MD simulations is very time consuming and if eliminated could vastly speed up the process. Not to mention, it is more representative of a realistic cross-linking and densification method. It is also worth mentioning that these materials are often cured at very high temperatures, which could easily be accounted for with MD. In summary, the crosslinking and densification process could be employed at the same time with temperature considerations specific to the material being studied. The overall effect on material characteristics would likely be minimal, but the suggested method would be less subject to question.

Another improvement to the models discussed in this report would be the consideration of atomic charges within the model. Atomic Charges would influence the crosslinking mechanics during the simulations, along with the behavior of the oxygen species attached to the oxidized graphene surfaces. Again, the overall influence on the end product would likely be minimal.

\subsection{Modeling Functionalized Graphene and Surface Treatments}

The existence of oxygen molecules on the surface of graphite structures enables for the bonding of larger molecules, which could then bond to the surrounding epoxy/hardener molecules. This process is known as functionalization,

and has been seen to produce significant improvements in composite properties. ${ }^{65-67}$ Molecular Dynamics models have been developed for a variety of functionalization 
methods. ${ }^{63,64,68-70}$ To fully understand the interactions between polymer matrix and carbon reinforcement, models should be developed for every possible scenario that can occur in nature. As the interfacial mechanisms become more understood, models can be developed that incorporate all of the interactions that may occur (nonbonded Van der Waals forces, charges from oxygen and nitrogen species, and bonding between carbon surface and surrounding polymer matrix). As the models become more accurate (realistic), they will become a more reliable tool for developing materials for very specific applications.

The use of MD could also be used to model various surface treatments that are performed during fabrication of polymer/carbon composites. Experimental research has revealed certain treatments to be beneficial to overall composite mechanical properties. These include: ultrasonic treatment ${ }^{71}$, plasma oxidation $^{72}$, electron beam irradiation ${ }^{73}$, and $\gamma$-ray irradiation ${ }^{74}$. The reason that these treatments work is because they alter the molecular behavior of the materials, something that can only be captured by MD.

\subsection{Modeling Aging in Composites}

Epoxy composites can often be subject to environments that promote both physical and chemical aging. Physical aging occurs when materials are exposed to temperatures below the glass transition for extended periods of time. This can cause a reduction in creep for composite materials. ${ }^{75}$ Chemical aging involves photochemical, oxidative, or thermal degradation which occurs due to formation or breaking of covalent bonds within a material. The most common form of chemical aging for composites is hygrothermal, which occurs in moist environments at high temperatures. ${ }^{76}$ Hygrothermal aging severs chemical bonds through hydrolysis and can result in loss of adhesion between epoxy and fiber. 
Molecular Dynamics has been used to model physical aging of the EPON 862/DETDA polymer system ${ }^{20}$, though it has never been investigated for a composite material. MD could be particularly useful in investigating the hygrothermal degradation process for an epoxy composite. It would be relatively easy to develop a composite model and then insert water molecules and increase the simulation temperature. This could be done for a variety of fiber/matrix systems.

The possibilities for research in the realm of MD are endless. Models will continue to become larger and more accurate as computational power increases and experimental techniques continue to reveal new information. 


\section{REFERENCES}

1. Drzal, L.T. and M. Madhukar, Fiber Matrix Adhesion and Its Relationship to Composite Mechanical-Properties. Journal of Materials Science, 1993. 28(3): p. 569-610.

2. Wagner, D.H. and R.A. Vaia, Nanocomposites: Issues at the Interface. Materials Today, 2004. 7(11): p. 38-42.

3. Potter, W. G., Epoxide Resins. In Springer-Verlag: New York, 1970.

4. Bascom, W.D. and Drzal, L.T., The Surface Properties of Carbon Fibers and Their Adhesion to Organic Polyers. NASA Contractor Report 4084, July 1987.

5. Drzal, L., The interphase in epoxy composites, in Epoxy Resins and Composites II, K. Dušek, Editor. 1986, Springer Berlin Heidelberg. p. 1-32.

6. Gojny, F.H., et al., Carbon nanotube-reinforced epoxy-composites: enhanced stiffness and fracture toughness at low nanotube content. Composites Science and Technology, 2004. 64(15): p. 2363-2371.

7. Gojny, F.H., et al., Influence of different carbon nanotubes on the mechanical properties of epoxy matrix composites - A comparative study. Composites Science and Technology, 2005. 65(15-16): p. 2300-2313.

8. RamanathanT, et al., Functionalized graphene sheets for polymer nanocomposites. Nat Nano, 2008. 3(6): p. 327-331.

9. Rafiee, M.A., et al., Enhanced Mechanical Properties of Nanocomposites at Low Graphene Content. ACS Nano, 2009. 3(12): p. 3884-3890.

10. King, J.A., et al., Mechanical properties of graphene nanoplatelet/epoxy composites. Journal of Applied Polymer Science, 2013. 128(6): p. 4217-4223.

11. Mäder, E. and E. Pisanova, Interfacial design in fiber reinforced polymers. Macromolecular Symposia, 2001. 163(1): p. 189-212.

12. LAMMPS website: http://lammps.sandia.gov

13. Stevens, M. J. Macromolecules 2001, 34, (8), 2710-2718.

14. Tsige, M.; Stevens, M. J. Macromolecules 2004, 37, (2), 630-637.

15. Carmesin, I.; Kremer, K. Macromolecules 1988, 21, (9), 2819-2823.

16. Jo, W. H.; Ko, M. B. Macromolecules 1994, 27, (26), 7815-7824.

17. Jo, W. H.; Ko, M. B. Macromolecules 1993, 26, (20), 5473-5478. 
18. Fan, H.B. and M.M.F. Yuen, Material properties of the cross-linked epoxy resin compound predicted by molecular dynamics simulation. Polymer, 2007. 48(7): p. 2174-2178.

19. Varshney, V., S. Patnaik, A. Roy, and B. Farmer, A Molecular Dynamics Study of Epoxy Based Networks: Cross-linking Procedure and Prediction of Molecular and Material Properties. Macromolecules, 2008. 41(18): p. 6837-6842

20. Bandyopadhyay, A., P.K. Valavala, T.C. Clancy, K.E. Wise, and G.M. Odegard, Molecular modeling of crosslinked epoxy polymers: The effect of crosslink density on thermomechanical properties. Polymer, 2011. 52(11): p. 2445-2452

21. Li, C.Y. and A. Strachan, Molecular dynamics predictions of thermal and mechanical properties of thermoset polymer EPON862/DETDA. Polymer, 2011. 52(13): p. 2920-2928

22. Frankland, S.J.V., A. Caglar, D.W. Brenner, and M. Griebel, Molecular Simulation of the Influence of Chemical Cross-Links on the Shear Strength of Carbon Nanotube-Polymer Interfaces. Journal of Physical Chemistry B, 2002. 106(12): p. 3046-3048

23. Odegard, G.M., T.S. Gates, K.E. Wise, C. Park, and E.J. Siochi, Constitutive modeling of nanotube-reinforced polymer composites. Composites Science and Technology, 2003. 63(11): p. 1671-1687.

24. Frankland, S.J.V., V.M. Harik, G.M. Odegard, D.W. Brenner, and T.S. Gates, The Stress-Strain Behavior of Polymer-Nanotube Composites from Molecular Dynamics Simulation. Composites Science and Technology, 2003. 63(11): p. 1655-1661.

25. Clancy, T.C. and T.S. Gates, Modeling of interfacial modification effects on thermal conductivity of carbon nanotube composites. Polymer, 2006. 47(16): p. 5990-5996.

26. Zhu, R., E. Pan, reinforced Epon 862 composites. Materials Science and Engineering a-Structural Materials Properties and A.K. Roy, Molecular dynamics study of the stress-strain behavior of carbon-nanotube Microstructure and Processing, 2007. 447(1-2): p. 51-57.

27. Nouranian, S., C. Jang, T.E. Lacy, S.R. Gwaltney, H. Toghiani, and C.U. Pittman, Molecular dynamics simulations of vinyl ester resin monomer interactions with a pristine vapor-grown carbon nanofiber and their implications for composite interphase formation. Carbon, 2011. 49(10): p. 3219-3232. 
28. Gou, J., et al., Computational and experimental study of interfacial bonding of single-walled nanotube reinforced composites. Computational Materials Science, 2004. 31(3-4): p. 225-236.

29. Odegard, G.M., T.C. Clancy, and T.S. Gates, Modeling of the mechanical properties of nanoparticle/polymer composites. Polymer, 2005. 46(2): p. 553-562.

30. Yu, S., S. Yang, and M. Cho, Multi-scale modeling of cross-linked epoxy nanocomposites. Polymer, 2009. 50(3): p. 945-952.

31. Jang, C., et al., Interfacial shear strength of cured vinyl ester resin-graphite nanoplatelet from molecular dynamics simulations. Polymer, 2013. 54(13): p. 32823289

32. Mansfield, K.F. and D.N. Theodorou, Atomistic Simulation of a Glassy Polymer Graphite Interface. Macromolecules, 1991. 24(15): p. 4295-4309

33. Stevens, M.J., Interfacial Fracture between Highly Cross-Linked Polymer Networks and a Solid Surface: Effect of Interfacial Bond Density. Macromolecules, 2001. 34: p. $2710-2718$.

34. Alkhateb, H., A. Al-Ostaz, and A. H. D. Cheng. "Molecular dynamics simulations of graphite-vinyl ester nanocomposites and their constituents. " Carbon Lett 11.4 (2010): 316-324.

35. Hadden, C.M., et al., Molecular modeling of EPON-862/graphite composites: Interfacial characteristics for multiple crosslink densities. Composites Science and Technology, 2013. 76(0): p. 92-99.

36. Gao, J.-S., S.-C. Shiu, and J.-L. Tsai, Mechanical properties of polymer near graphite sheet. Journal of Composite Materials, 2013. 47(4): p. 449-458.

37. Li, C., et al., Atomistic simulations on multilayer graphene reinforced epoxy composites. Composites Part A: Applied Science and Manufacturing, 2012. 43(8): p. 1293-1300.

38. Mittal, R., et al. Molecular modeling for calculation of mechanical properties of EPON862 / SWCNTs composites. in Nanoscience, Engineering and Technology (ICONSET), 2011 International Conference on. 2011.

39. Han, Y. and J. Elliott, Molecular dynamics simulations of the elastic properties of polymer/carbon nanotube composites. Computational Materials Science, 2007. 39(2): p. 315-323. 
40. Ionita, M., Multiscale molecular modeling of SWCNTs/epoxy resin composites mechanical behaviour. Composites Part B: Engineering, 2012. 43(8): p. 3491-3496.

41. Duffy, E. M.; Kowalczyk, P. J.; Jorgensen, W. L. Journal of the American Chemical Society 1993, 115, (20), 9271-9275.

42. Jorgensen, W. L.; Maxwell, D. S.; TiradoRives, J. Journal of the American Chemical Society 1996, 118, (45), 11225-11236.

43. Weiner, S. J.; Kollman, P. A.; Case, D. A.; Singh, U. C.; Ghio, C.; Alagona, G.; Profeta, S.; Weiner, P. Journal of the American Chemical Society 1984, 106, (3), 765-784.

44. Watkins, E. K.; Jorgensen, W. L. Journal of Physical Chemistry A 2001, 105, (16), 4118-4125.

45. Plimpton, S., Fast Parallel Algorithms for Short-Range Molecular-Dynamics Journal of Computational Physics, 1995. 117(1): p. 1-19.

46. Hoover, W.G., Canonical Dynamics - Equilibrium Phase-Space Distributions. Physical Review A, 1985. 31(3): 1695-1697.

47. Yarovsky, I. and E. Evans, Computer simulation of structure and properties of crosslinked polymers: application to epoxy resins. Polymer, 2002. 43(3): 963-969.

48. Littell, J.D., C.R. Ruggeri, R.K. Goldberg, G.D. Roberts, W.A. Arnold, and W.K. Binienda, Measurement of epoxy resin tension, compression, and shear stress-strain curves over a wide range of strain rates using small test specimens. Journal of Aerospace Engineering, 2008. 21(3): p. 162-173.

49. Kuilla, T., et al., Recent advances in graphene based polymer composites. Progress in Polymer Science, 2010. 35(11): p. 1350-1375.

50. Tang, L.-C., et al., The effect of graphene dispersion on the mechanical properties of graphene/epoxy composites. Carbon, 2013. 60(0): p. 16-27

51. Aboudi, J.; Arnold, S. M.; Bednarcyk, B. A. (2013): Micromechanics of Composite Materials: A Generalized Multiscale Analysis Approach. Elsevier, Inc.

52. Bednarcyk, B.A., J. Aboudi, and S.M. Arnold, The effect of general statistical fiber misalignment on predicted damage initiation in composites. Composites Part B: Engineering, 2014. 66(0): p. 97-108.

53. Mechanics of Materials, 1992. 14(2): p. 127-139.Paley, M. and J. Aboudi, Micromechanical analysis of composites by the generalized cells model. 
54. HexTow ${ }^{\circledR}$ Continuous Carbon Fiber Product Literature; Hexcel: Stamford, CT (2011)

55. Eda, G., et al., Partially oxidized graphene as a precursor to graphene. Journal of Materials Chemistry, 2011. 21(30): p. 11217-11223.

56. Szabó, T., et al., Evolution of Surface Functional Groups in a Series of

Progressively Oxidized Graphite Oxides. Chemistry of Materials, 2006. 18(11): p. 2740-2749

57. Dreyer, D.R., et al., The chemistry of graphene oxide. Chemical Society Reviews, 2010. 39(1): p. 228-240.

58. Allington, R.D., et al., A model of the surface of oxidatively treated carbon fibre based on calculations of adsorption interactions with small molecules. Composites Part A: Applied Science and Manufacturing, 1998. 29(9-10): p. 1283-1290.

59. Allington, R.D., et al., Developing improved models of oxidatively treated carbon fibre surfaces, using molecular simulation. Composites Part A: Applied Science and Manufacturing, 2004. 35(10): p. 1161-1173.

60. Kudin, K.N., et al., Raman Spectra of Graphite Oxide and Functionalized Graphene Sheets. Nano Letters, 2007. 8(1): p. 36-41.

61. Boukhvalov, D.W. and M.I. Katsnelson, Modeling of Graphite Oxide. Journal of the American Chemical Society, 2008. 130(32): p. 10697-10701.

62. Sadiq Awan, I., et al., Developing an approach to calculate carbon fiber surface energy using molecular simulation and its application to real carbon fibers. Journal of Composite Materials, 2011.

63. Xiaoqun, W., et al., Molecular dynamics simulations of interfacial adhesion between carbon fibers and various epoxies/hardeners and its calorimetric validation. Journal of Composite Materials, 2013. 47(8): p. 1011-1017.

64. Rahman, R. and A. Haque, Molecular modeling of crosslinked graphene-epoxy nanocomposites for characterization of elastic constants and interfacial properties. Composites Part B: Engineering, 2013. 54(0): p. 353-364.

65. Ganguli, S., A.K. Roy, and D.P. Anderson, Improved thermal conductivity for chemically functionalized exfoliated graphitelepoxy composites. Carbon, 2008. 46(5): p. 806-817. 
66. Miller, S.G., et al., Characterization of epoxy functionalized graphite nanoparticles and the physical properties of epoxy matrix nanocomposites. Composites Science and Technology, 2010. 70(7): p. 1120-1125.

67. Mäder, E. and E. Pisanova, Interfacial design in fiber reinforced polymers. Macromolecular Symposia, 2001. 163(1): p. 189-212

68. Attwood, D. and P.I. Marshall, Atomistic modelling of the adsorption of epoxy and amine molecules on the surface of carbon fibres. Composites Part A: Applied Science and Manufacturing, 1996. 27(9): p. 775-779.

69. Sanchez, F. and L. Zhang, Molecular dynamics modeling of the interface between surface functionalized graphitic structures and calcium-silicate-hydrate: Interaction energies, structure, and dynamics. Journal of Colloid and Interface Science, 2008. 323(2): p. 349-358.

70. Aparicio, S. and M. Atilhan, Choline-Based Ionic Liquids on Graphite Surfaces and Carbon Nanotubes Solvation: A Molecular Dynamics Study. The Journal of Physical Chemistry C, 2012. 116(22): p. 12055-12065.

71. Huang, Y.D., et al., Influence of ultrasonic treatment on the characteristics of epoxy resin and the interfacial property of its carbon fiber composites. Composites Science and Technology, 2002. 62(16): p. 2153-2159.

72. Montes-Morán, M.A., et al., Effects of plasma oxidation on the surface and interfacial properties of carbon fibres/polycarbonate composites. Carbon, 2001. 39(7): p. 1057-1068.

73. Zhang, Z., et al., The effect of carbon-fiber surface properties on the electron-beam curing of epoxy-resin composites. Composites Science and Technology, 2002. 62(3): p. 331-337.

74. Xu, Z., et al., Effect of $\gamma$-ray irradiation grafting on the carbon fibers and interfacial adhesion of epoxy composites. Composites Science and Technology, 2007. 67(1516): p. 3261-3270.

75. Sullivan, J.L., Creep and physical aging of composites. Composites Science and Technology, 1990. 39(3): p. 207-232

76. Ray, B.C., Temperature effect during humid ageing on interfaces of glass and carbon fibers reinforced epoxy composites. Journal of Colloid and Interface Science, 2006. 298(1): p. 111-117. 


\section{APPENDIX A}

\section{LAMMPS scripts for MD simulations, MM simulations, and deformation}

simulations

\section{A.1. Molecular Energy Minimization Script}

\#Energy minimisation input script

units real

dimension 3

boundary $\mathrm{p} \mathrm{p} \mathrm{p}$

atom_style molecular

neighbor 2.0 bin

neigh_modify one 25000 page 250000

echo screen

\#OPLS potentials

bond_style harmonic

angle_style harmonic

dihedral_style opls

pair_style lj/cut/opt 10.0

pair_modify mix arithmetic

read_data filename.xyz

\#GROUPS AND FIXES (FROM MODELS IN CHAPTER 3)

group plug id > 21120

group graphite type 14

fix 1 plug setforce 0.00 .00 .0

fix 2 graphite setforce 0.00 .00 .0

neigh_modify exclude group plug plug

neigh_modify exclude group graphite graphite

dump 1 all xyz 500 dump.filename.xyz

dump 2 all atom 500 dump.filename.lammpstrj

dump_modify 2 scale no

\#minimisation

min_style cg \#conjugate gradient

minimize 0.00 .010000100000

write_restart min.filename.restart 


\section{A.2. Molecular Dynamics NVT simulation script for equilibration \# NVT simulation for equilibration}

units real

dimension 3

boundary $\mathrm{p} \mathrm{p} \mathrm{p}$

atom_style molecular

neighbor 5.0 bin

neigh_modify every 1 delay 10 one 4000 page 250000

echo screen

\#-----------------OPLS potentials---------------------

bond_style harmonic

angle_style harmonic

dihedral_style opls

pair_style lj/cut/opt 10.0

pair_modify mix arithmetic

read_restart min.filename.restart

\#---------Define Groups for specific output---------

group graphite type 14161718

group benzene type 18

\#group xlink type 1617181920

\#------Commands for freezing atoms

\#neigh_modify exclude group plug plug

\#neigh_modify exclude group graphite graphite

\#----------------initial velocities---------------------------------

velocity all create 300.03094152

compute 1 all temp

compute 2 all pe

\#-----per-atom potential energy and virial contributions-------

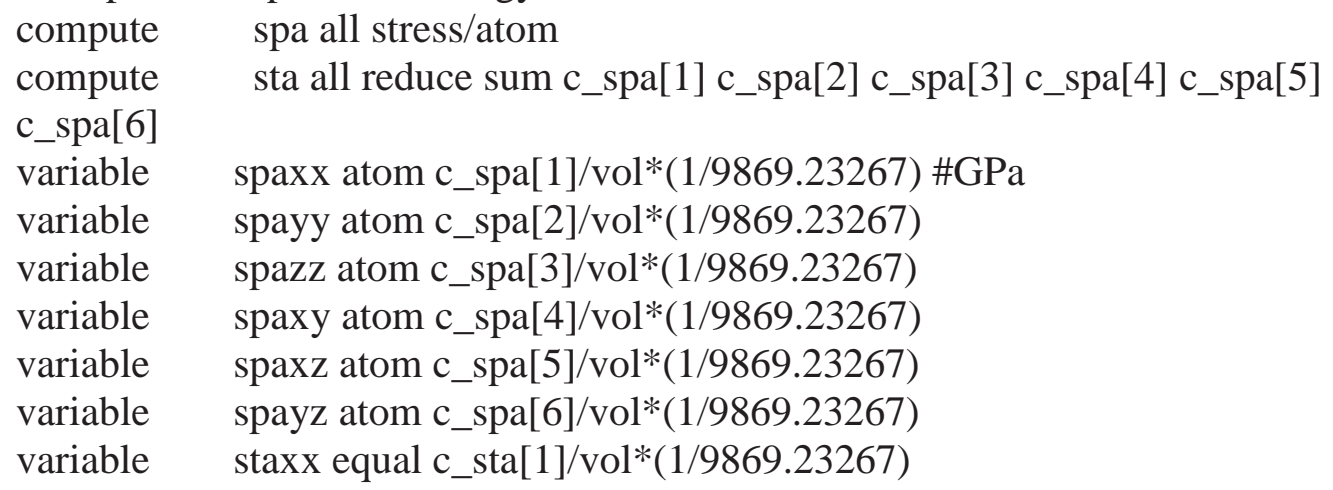


variable stayy equal c_sta[2]/vol*(1/9869.23267)

variable stazz equal c_sta[3]/vol*(1/9869.23267)

variable staxy equal c_sta[4]/vol*(1/9869.23267)

variable staxz equal c_sta[5]/vol*(1/9869.23267)

variable stayz equal c_sta[6]/vol*(1/9869.23267)

variable spaVM atom sqrt((v_spaxx-v_spayy $)^{\wedge} 2+\left(\mathrm{v} \_\right.$spayy-

v_spazz $\left.)^{\wedge} 2+\left(\mathrm{v} \_s p a x x-v \_s p a z z\right)^{\wedge} 2+6^{*}\left(\mathrm{v} \_s p a x y^{\wedge} 2+\mathrm{v} \_s p a x z^{\wedge} 2+\mathrm{v} \_s p a y z^{\wedge} 2\right)\right)$ \# von

Mises stress

compute ppa all pe/atom

compute pta all reduce ave c_ppa

variable ppa atom c_ppa

\#compute ppavdw all pe/atom pair

variable ppavdw atom c_ppavdw

\#compute ppabnd all pe/atom bond

\#compute ppaang all pe/atom angle

\#compute ppadih all pe/atom dihedral

\#-------fixes for calculating various denity, stress, and energy contributions------

fix 1 all nvt temp 300.0300 .010 .0

\#--------density calculations along $\mathrm{z}$-axis-----------------

fix 2 all ave/spatial $50050500000 \mathrm{z}$ lower 0.1 density/mass units box ave one file density1.504.c75.eq.txt

\#--------densification command

fix 3 all deform $1 \mathrm{z}$ final -16.1516 .15 units box

\#--------specific outputs - alter as desired----------------

\#fix 5 benzene ave/spatial 5005025000 z lower 0.2 density/mass units box ave one file density.filename.benzene.txt

\#fix 6 all ave/spatial $111 \mathrm{z}$ lower 0.1 c_ppa c_ppavdw c_ppabnd c_ppaang

c_ppadih units box ave one file energy.filename.txt

\#fix 6 all ave/spatial 5005025000 z lower 0.1 c_ppa c_ppavdw c_ppabnd c_ppaang

c_ppadih units box ave one file energy.filename.txt

\#fix 7 all ave/spatial $111 \mathrm{z}$ lower $0.1 \mathrm{v} \_s p a x x$ v_spayy v_spazz v_spaxz v_spaxy

v_spayz v_spalen units box ave one file stress.filename.txt

fix 4 all ave/spatial $5005050000 \mathrm{z}$ lower 0.1 v_spaxx v_spayy v_spazz v_spaxz

v_spaxy v_spayz v_spalen units box ave one file stress.filename.txt

\#fix 8 benzene ave/spatial 5005025000 z lower 0.1 v_spaxx v_spayy v_spazz

v_spaxz v_spaxy v_spayz v_spalen units box ave one file stress.filename.benzene.txt

\#----OVITO stress and energy dump files for visualization---

\#dump normal all custom 500000 stress.filename.lammpstrj id type x y z v_spaxx

v_spayy v_spazz v_spaVM

\#dump_modify normal sort id flush yes

\#dump shear all custom 5000 shear.filename.lammpstrj id type x y z v_spaxy

v_spaxz v_spayz

\#dump_modify shear sort id flush yes 
dump pe_st graphite custom 50000 Graphite.energy.lammpstrj id type x y z v_ppa dump_modify pe_st sort id flush yes

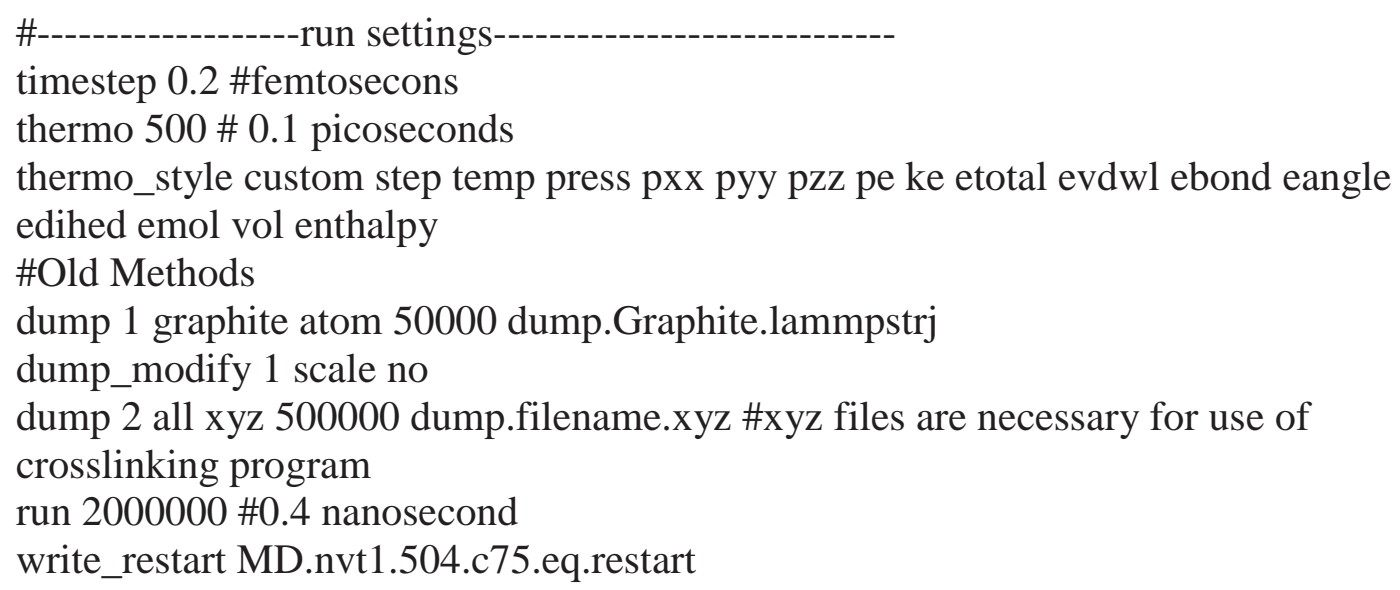




\section{A.3. Molecular Dynamics NPT simulation script for Tensile Deformation} \# NPT Simulation for Tensile Deformation

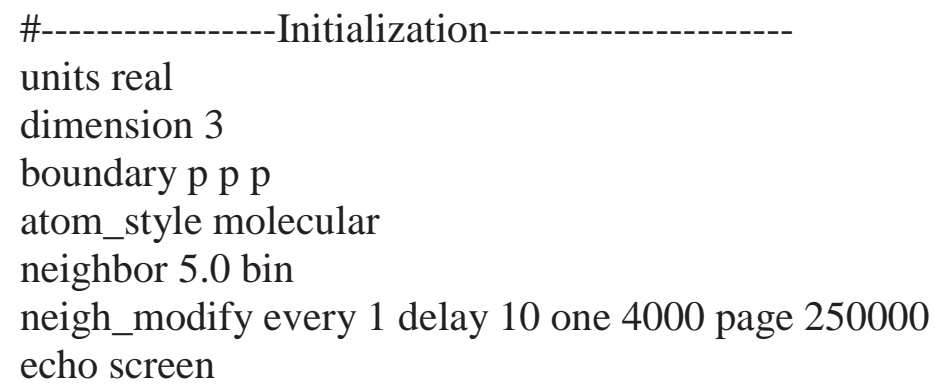




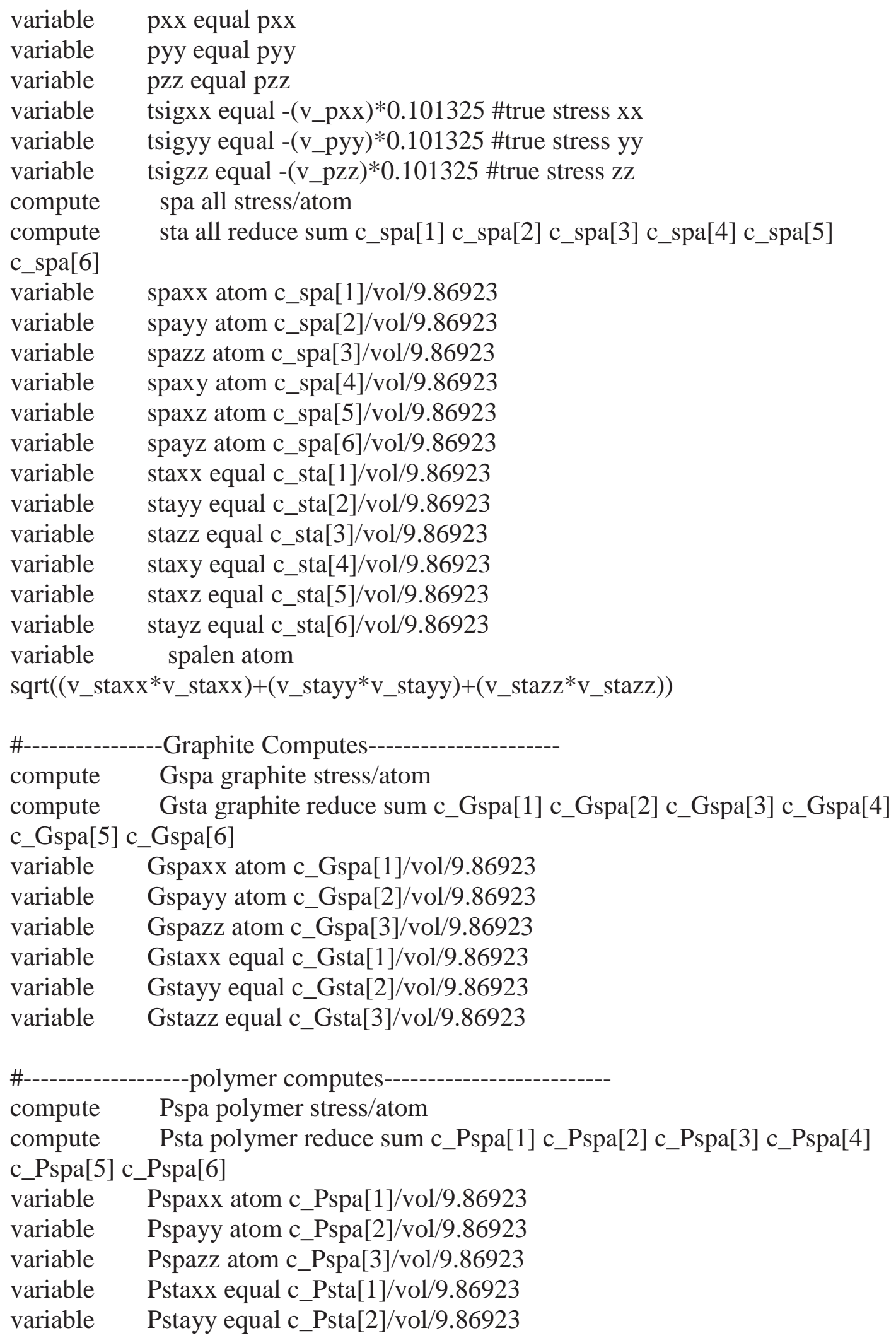


variable Pstazz equal c_Psta[3]/vol/9.86923

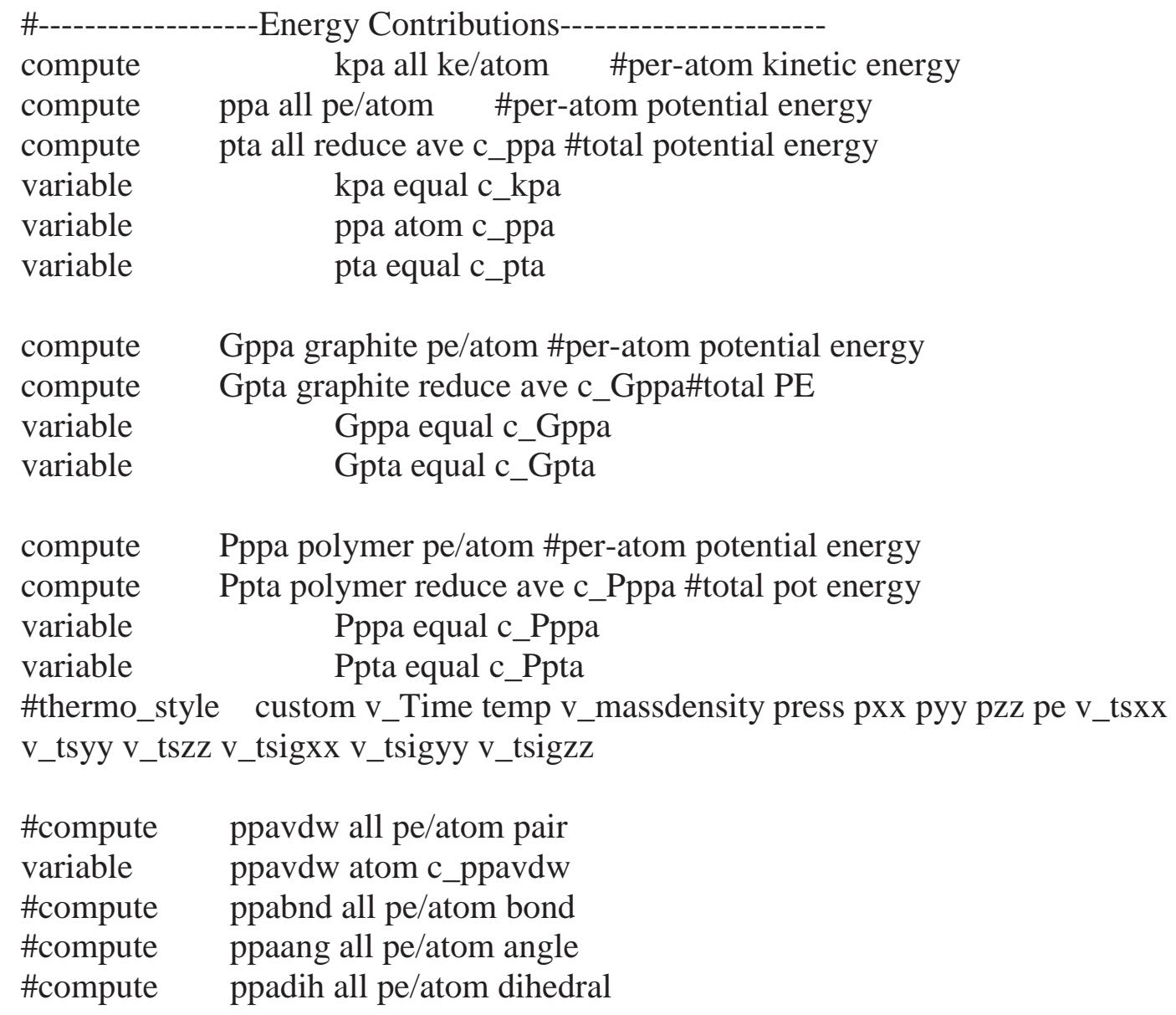


dump_modify pe_st sort id flush yes

\#-----------------run settings----------------------------

thermo_style custom step v_Time temp vol pzz pxx pyy ke pe etotal v_pta v_Gpta v_Ppta

timestep $0.2 \#$ femtosecons

thermo 1000 \# 0.2 picoseconds

\#thermo_style custom step temp press pxx pyy pzz pe ke etotal evdwl ebond eangle edihed emol vol enthalpy

\#-------------dump files for visualization---------

dump 1 all atom 500000 dump.nvt1.504.c75.Exx.lammpstrj

dump_modify 1 scale no

dump 2 all xyz 1000000 dump.nvt1.504.c75.Exx.xyz

run 5000000 \# 1 nanosecond

write_restart deformed1.504.c75.Exx.restart 


\section{A.4. Molecular Dynamics NVT simulation script for Shear Deformation}

\# NVT simulation for Shear Deformation

\#------------------Initialization-----------------------

Same as A.3

\#-----------------AMBER potentials---------------------

Same as A.3

\#--------------initial velocities--------------------------------

Same as A.3

\#-----per-atom potential energy and virial contributions-------

Same as A.3

\#------MAIN DEFORMATION COMMAND USE NVT FOR SHEAR---------------

fix 1 all nvt temp 300.0300 .010 .0

fix 5 all deform 1 xy final 5.0 remap none units box \#DEFORM 5 ANGSTROMS

$\sim 5 \%$ OF X AND Y BOX SIZE 100Angstroms

\#---SHEAR DATA OUTPUT COMMAND FOR STRESS STRAIN CURVES---

fix 7 all ave/time 11001000 v_xy v_yz v_xz v_staxy v_stayz v_staxz file

Gxy.shear.filename.txt

\#------OVITO stress and energy dump files

dump normal all custom 500000 Gxy.stress.lammpstrj id type x y z v_spaxx v_spayy

v_spazz v_spalen

dump_modify normal sort id flush yes

dump shear all custom 500000 Gxy.shear.lammpstrj id type x y z v_spaxy v_spaxz

v_spayz

dump_modify shear sort id flush yes

dump pe_st all custom 500000 Gxy.energy.lammpstrj id type x y z v_ppa

dump_modify pe_st sort id flush yes

\#--------------------run settings-----------------------------

thermo_style custom step v_Time temp vol pzz pxx pyy ke pe etotal v_pta v_Gpta

v_Ppta

timestep 0.2 \#femtosecons

thermo 1000 \# 0.2 picoseconds

\#thermo_style custom step temp press pxx pyy pzz pe ke etotal evdwl ebond eangle

edihed emol vol enthalpy

\#-------------------Dump commands for visualization-------------

dump 1 all atom 500000 dump.Gxy.filename.lammpstrj

dump_modify 1 scale no

dump 2 all xyz 1000000 dump.Gxy.filename.xyz

run 5000000 \# 1 nanosecond

write_restart Gxy.filename.restart 


\section{A.5. MD OPLS UA Parameters from Coordinates File \\ \#DATA FOR UNITED ATOM SYSTEM WITH 1 HYDROXYL AND EPOXIDE FUNCTIONALIZED GRAPHITE SHEET}

25857 atoms

23 atom types

29558 bonds

27 bond types

48419 angles

40 angle types

77086 dihedrals

56 dihedral types

-6.0599999999999998e-01 1.0053800000000000e+02 xlo xhi

-7.0999999999999996e-01 1.0356999999999999e+02 ylo yhi

$-1.6199999999999999 e+011.6199999999999999 e+01$ zlo zhi

Masses

atomid mass

1 12.011 \#benzene carbon

21.008 \#benzene hydrogen

314.027 \#CH2 bonded to ring

414.027 \#CH2 bonded to $\mathrm{O}$

515.9994 \#oxygen before $x$-link

6 13.017 \#CH ether

715.9994 \#oxygen bonded to ring

8 12.011 \#carbon bonded to $\mathrm{O}$

915.035 \#CH3

10 14.007 \#non-xlink nitrogen

111.008 \#hydrogen $x$-link

1212.0 \#carbon from $\mathrm{CH} 3$

131.008 \#hydrogen from $\mathrm{CH} 3$

14 12.0 \#graphene carbon

15 14.027 \#x-link CH2

1615.9994 \#functional hydroxyl O

171.008 \#functional hydroxyl $\mathrm{H}$

1815.9994 \#functional epoxide $\mathrm{O}$

19 14.007 \#nitrogen 1-xlink

2015.9994 \#crosslinked O

21 14.027 \#crosslinked CH2

221.008 \#crosslinked $\mathrm{H}$ 
23 14.007 \#nitrogen 2-xlinks

Bond Coeffs

bondtype $\mathrm{K} \mathrm{r0}$

16320.01 .425 \# $\mathrm{CH} 2$ bonded to $\mathrm{O}$ - oxygen bonded to ring

10317.01 .510 \#benzene carbon - $\mathrm{CH} 2$ bonded to ring

23434.01 .010 \#non-xlink nitrogen - hydrogen x-link

21320.01 .425 \#oxygen before $\mathrm{x}$-link - $\mathrm{CH}$ ether

2260.0 1.526 \#CH ether - crosslinked CH2

18434.01 .010 \#hydrogen x-link - nitrogen 1-xlink

8367.01 .080 \#benzene carbon - benzene hydrogen

6340.01 .090 \#carbon from CH3 - hydrogen from CH3

26450.01 .364 \#graphene carbon - functional epoxide $\mathrm{O}$

25469.0 1.400 \#graphene carbon - graphene carbon

22481.01 .340 \#benzene carbon - non-xlink nitrogen

20260.01 .526 \#CH ether - x-link CH2

7469.01 .400 \#benzene carbon - benzene carbon

17337.0 1.463 \#nitrogen 1-xlink - crosslinked CH2

24553.0 0.945 \#functional hydroxyl $\mathrm{O}$ - functional hydroxyl $\mathrm{H}$

5553.00 .945 \#crosslinked O - crosslinked H

9481.0 1.340 \#benzene carbon - nitrogen 2-xlinks

4337.0 1.463 \#crosslinked CH2 - nitrogen 2-xlinks

11481.01 .340 \#benzene carbon - nitrogen 1-xlink

27450.01 .364 \#graphene carbon - functional hydroxyl O

15260.01 .526 \#CH2 bonded to $\mathrm{O}-\mathrm{CH}$ ether

12469.01 .400 \#benzene carbon - carbon from $\mathrm{CH} 3$

3386.0 1.425 \#CH ether - crosslinked O

13 469.0 1.400 \#benzene carbon - carbon bonded to $\mathrm{O}$

1260.01 .526 \#CH2 bonded to ring - $\mathrm{CH} 3$

19320.01 .425 \# oxygen before x-link - x-link CH2

14450.01 .364 \#oxygen bonded to ring - carbon bonded to $\mathrm{O}$

Angle Coeffs

angletype $\mathrm{K}$ theta0

17 80.0 109.5 \#CH2 bonded to $\mathrm{O}$ - $\mathrm{CH}$ ether - crosslinked $\mathrm{O}$

37 35.0 118.4 \#hydrogen x-link - nitrogen 1-xlink - crosslinked CH2

14 63.0 112.4 \#benzene carbon - $\mathrm{CH} 2$ bonded to ring - benzene carbon

26 80.0 109.5 \#crosslinked O - CH ether - crosslinked CH2

1535.0 109.5 \#benzene carbon - carbon from CH3 - hydrogen from $\mathrm{CH} 3$

38 43.6 106.4 \#hydrogen x-link - non-xlink nitrogen - hydrogen x-link

2080.0 109.5 \#CH ether - $\mathrm{CH} 2$ bonded to $\mathrm{O}$ - oxygen bonded to ring

12 63.0 114.0 \#benzene carbon - $\mathrm{CH} 2$ bonded to ring - $\mathrm{CH} 3$

185.0120 .0 \#graphene carbon - graphene carbon - graphene carbon

3235.0111 .0 \#benzene carbon - nitrogen 1-xlink - hydrogen x-link

25 85.0 120.0 \#benzene carbon - benzene carbon - carbon bonded to $\mathrm{O}$ 
835.0120 .0 \#benzene hydrogen - benzene carbon - carbon bonded to O 2980.0 111.2 \#CH ether - crosslinked CH2 - nitrogen 2-xlinks 2363.0 111.1 \#CH2 bonded to $\mathrm{O}$ - $\mathrm{CH}$ ether - x-link CH2 4070.0 120.0 \#benzene carbon - benzene carbon - non-xlink nitrogen 5100.0111 .8 \#CH2 bonded to $\mathrm{O}$ - oxygen bonded to ring - carbon bonded to $\mathrm{O}$ 433.0107 .8 \#hydrogen from $\mathrm{CH} 3$ - carbon from $\mathrm{CH} 3$ - hydrogen from $\mathrm{CH} 3$ 1150.0 123.2 \#benzene carbon - nitrogen 2-xlinks - crosslinked CH2 2 85.0 120.0 \#benzene carbon - benzene carbon - carbon from $\mathrm{CH} 3$ 19 80.0 111.2 \#CH ether - crosslinked CH2 - nitrogen 1-xlink 2135.0111 .0 \#benzene carbon - non-xlink nitrogen - hydrogen x-link 3050.0118 .0 \#crosslinked $\mathrm{CH} 2$ - nitrogen 2-xlinks - crosslinked $\mathrm{CH} 2$ 1685.0 120.0 \#benzene carbon - carbon bonded to $\mathrm{O}$ - benzene carbon 27 70.0 120.0 \#benzene carbon - benzene carbon - nitrogen 1-xlink 28 80.0 109.5 \# oxygen before x-link - x-link CH2 - CH ether 13 85.0 120.0 \#benzene carbon - benzene carbon - benzene carbon 24 80.0 109.5 \# oxygen before x-link - CH ether - x-link CH2 670.0120 .0 \#graphene carbon - graphene carbon - functional hydroxyl O 3355.0 108.5 \#graphene carbon - functional hydroxyl $\mathrm{O}$ - functional hydroxyl $\mathrm{H}$ 1070.0120 .0 \#benzene carbon - carbon bonded to $\mathrm{O}$ - oxygen bonded to ring 3970.0 120.0 \#graphene carbon - graphene carbon - functional epoxide O 370.0120 .0 \#benzene carbon - benzene carbon - $\mathrm{CH} 2$ bonded to ring 34 55.0 108.5 \#CH ether - crosslinked O - crosslinked $\mathrm{H}$ 3180.0109 .5 \#CH2 bonded to $\mathrm{O}$ - $\mathrm{CH}$ ether - oxygen before x-link 9 35.0 120.0 \#benzene carbon - benzene carbon - benzene hydrogen 3650.0 123.2 \#benzene carbon - nitrogen 1-xlink - crosslinked CH2 2270.0120 .0 \#benzene carbon - benzene carbon - nitrogen 2-xlinks 35 100.0 111.8 \#graphene carbon - functional epoxide O - graphene carbon 18 100.0 111.8 \#CH ether - oxygen before x-link - x-link CH2 763.0111 .1 \#CH2 bonded to $\mathrm{O}$ - $\mathrm{CH}$ ether - crosslinked $\mathrm{CH} 2$

\author{
Dihedral Coeffs \\ dihedraltype K1 K2 K3 K4 \\ 310.00 .0000 .0000 .0 \#benzene carbon - nitrogen 2-xlinks - crosslinked $\mathrm{CH} 2$ - $\mathrm{CH}$ ether \\ 400.02 .6500 .0000 .0 \#carbon from CH3 - benzene carbon - benzene carbon - nitrogen 1-xlink \\ 250.00 .2001 .450 0.0 \#benzene carbon - benzene carbon - carbon bonded to $\mathrm{O}$ - oxygen bonded to ring \\ 380.0 0.000 0.000 0.0 \#CH ether - crosslinked CH2 - nitrogen 2-xlinks - crosslinked CH2 \\ 40.02 .6500 .000 0.0 \#graphene carbon - graphene carbon - graphene carbon - graphene carbon \\ 290.01 .8000 .0000 .0 \# oxygen bonded to ring - $\mathrm{CH} 2$ bonded to $\mathrm{O}-\mathrm{CH}$ ether - crosslinked $\mathrm{O}$ \\ 80.01 .0002 .000 0.0 \#benzene carbon - carbon bonded to $\mathrm{O}$ - oxygen bonded to ring - $\mathrm{CH} 2$ bonded to $\mathrm{O}$ \\ 70.02 .6500 .000 0.0 \#benzene carbon - benzene carbon - benzene carbon - benzene carbon \\ 410.02 .6500 .000 0.0 \#benzene carbon - benzene carbon - nitrogen 1-xlink - hydrogen x-link \\ 360.02 .6500 .000 0.0 \#benzene carbon - benzene carbon - benzene carbon - nitrogen 2-xlinks \\ 340.02 .6500 .0000 .0 \#oxygen bonded to ring - $\mathrm{CH} 2$ bonded to $\mathrm{O}-\mathrm{CH}$ ether - crosslinked $\mathrm{CH} 2$ \\ 10.00 .2001 .4500 .0 \#CH ether - $\mathrm{CH} 2$ bonded to $\mathrm{O}$ - oxygen bonded to ring - carbon bonded to $\mathrm{O}$ \\ 100.02 .6500 .000 0.0 \#benzene carbon - benzene carbon - $\mathrm{CH} 2$ bonded to ring - benzene carbon \\ 60.00 .2001 .450 0.0 \#graphene carbon - graphene carbon - functional epoxide $\mathrm{O}$ - graphene carbon \\ 140.01 .8000 .0000 .0 \#benzene hydrogen - benzene carbon - carbon bonded to $\mathrm{O}$ - oxygen bonded to ring \\ 120.02 .6500 .0000 .0 \#benzene hydrogen - benzene carbon - benzene carbon - $\mathrm{CH} 2$ bonded to ring \\ 480.01 .8000 .000 0.0 \# oxygen before $\mathrm{x}$-link - $\mathrm{CH}$ ether - $\mathrm{CH} 2$ bonded to $\mathrm{O}$ - oxygen bonded to ring \\ 50.01 .0002 .000 0.0 \#graphene carbon - graphene carbon - graphene carbon - functional hydroxyl O
}


460.00 .001 .0000 .0 \#CH2 bonded to $\mathrm{O}$ - $\mathrm{CH}$ ether - crosslinked $\mathrm{O}$ - crosslinked $\mathrm{H}$ 550.00 .0000 .000 0.0 \#benzene carbon - nitrogen 1-xlink - crosslinked CH2 - CH ether 350.02 .6500 .0000 .0 \#carbon from $\mathrm{CH} 3$ - benzene carbon - benzene carbon - nitrogen 2-xlinks 430.00 .2001 .4500 .0 \#CH2 bonded to $\mathrm{O}$ - $\mathrm{CH}$ ether - oxygen before $\mathrm{x}$-link - $\mathrm{x}$-link $\mathrm{CH} 2$ 150.02 .6500 .0000 .0 \#benzene carbon - benzene carbon - benzene carbon - carbon bonded to $\mathrm{O}$ 220.02 .6500 .0000 .0 \#benzene hydrogen - benzene carbon - benzene carbon - carbon bonded to $\mathrm{O}$ 90.02 .6500 .000 0.0 \# oxygen bonded to ring - $\mathrm{CH} 2$ bonded to $\mathrm{O}-\mathrm{CH}$ ether - $\mathrm{x}$-link $\mathrm{CH} 2$ 20.01 .8000 .000 0.0 \#functional hydroxyl O - graphene carbon - graphene carbon - functional hydroxyl $\mathrm{O}$ 540.00 .2001 .4500 .0 \#graphene carbon - functional epoxide $\mathrm{O}$ - graphene carbon - graphene carbon 190.02 .6500 .000 0.0 \#benzene carbon - benzene carbon - benzene carbon - benzene hydrogen 440.00 .0000 .9240 .0 \#benzene carbon - benzene carbon - carbon from $\mathrm{CH} 3$ - hydrogen from $\mathrm{CH} 3$ 170.01 .0002 .000 0.0 \#benzene carbon - carbon bonded to $\mathrm{O}$ - benzene carbon - benzene hydrogen 30.02 .6500 .000 0.0 \#benzene hydrogen - benzene carbon - benzene carbon - benzene hydrogen 130.02 .6500 .0000 .0 \#benzene carbon - carbon bonded to $\mathrm{O}$ - benzene carbon - benzene carbon 560.02 .6500 .0000 .0 \# $\mathrm{CH} 2$ bonded to ring - benzene carbon - benzene carbon - nitrogen 1-xlink 370.01 .0002 .0000 .0 \#CH2 bonded to $\mathrm{O}$ - $\mathrm{CH}$ ether - $\mathrm{x}$-link $\mathrm{CH} 2$ - oxygen before $\mathrm{x}$-link 210.02 .6500 .0000 .0 \#benzene carbon - benzene carbon - benzene carbon - carbon from $\mathrm{CH} 3$ 510.00 .004 .000 .0 \#CH2 bonded to $\mathrm{O}$ - $\mathrm{CH}$ ether - crosslinked $\mathrm{CH} 2$ - nitrogen 1-xlink 180.00 .001 .0000 .0 \#graphene carbon - graphene carbon - functional hydroxyl $\mathrm{O}$ - functional hydroxyl $\mathrm{H}$ 270.02 .6500 .0000 .0 \#benzene carbon - $\mathrm{CH} 2$ bonded to ring - benzene carbon - benzene carbon 520.02 .6500 .000 0.0 \#benzene carbon - benzene carbon - non-xlink nitrogen - hydrogen x-link 490.02 .6500 .000 0.0 \#non-xlink nitrogen - benzene carbon - benzene carbon - carbon from $\mathrm{CH} 3$ 500.02 .6500 .0000 .0 \#benzene carbon - benzene carbon - $\mathrm{CH} 2$ bonded to ring - $\mathrm{CH} 3$

160.02 .6500 .0000 .0 \#benzene carbon - benzene carbon - benzene carbon - $\mathrm{CH} 2$ bonded to ring 530.01 .8000 .0000 .0 \#functional hydroxyl O - graphene carbon - graphene carbon - functional epoxide $\mathrm{O}$ 330.013 .60 .0000 .0 \#benzene carbon - benzene carbon - nitrogen 1-xlink - crosslinked CH2 30 0.0 2.650 0.000 0.0 \#benzene carbon - benzene carbon - carbon bonded to $\mathrm{O}$ - benzene carbon $320.02 .6500 .0000 .0 \# \mathrm{CH} 2$ bonded to ring - benzene carbon - benzene carbon - non-xlink nitrogen 230.00 .001 .000 0.0 \#crosslinked $\mathrm{CH} 2$ - $\mathrm{CH}$ ether - crosslinked $\mathrm{O}$ - crosslinked $\mathrm{H}$ 420.0 0.00 4.00 0.0 \#nitrogen 1-xlink - crosslinked CH2 - CH ether - crosslinked O 200.00 .004 .000 .0 \#crosslinked $\mathrm{O}$ - $\mathrm{CH}$ ether - crosslinked $\mathrm{CH} 2$ - nitrogen 2-xlinks 470.02 .6500 .0000 .0 \#CH2 bonded to ring - benzene carbon - benzene carbon - nitrogen 2-xlinks 110.01 .0002 .000 0.0 \#graphene carbon - graphene carbon - graphene carbon - functional epoxide $\mathrm{O}$ 390.02 .650 0.000 0.0 \#benzene carbon - benzene carbon - benzene carbon - nitrogen 1-xlink 260.00 .004 .000 .0 \#CH2 bonded to $\mathrm{O}-\mathrm{CH}$ ether - crosslinked $\mathrm{CH} 2$ - nitrogen 2-xlinks 280.00 .0000 .0000 .0 \#CH ether - crosslinked CH2 - nitrogen 1-xlink - hydrogen x-link 450.02 .6500 .0000 .0 \#benzene carbon - benzene carbon - benzene carbon - non-xlink nitrogen 240.013 .60 .000 0.0 \#benzene carbon - benzene carbon - nitrogen 2-xlinks - crosslinked $\mathrm{CH} 2$

Pair Coeffs

epsilon sigma

10.07003 .5500 \#benzene carbon

20.03002 .4200 \#benzene hydrogen

30.1803 .9050 \#CH2 bonded to ring

40.11803 .8000 \#CH2 bonded to $\mathrm{O}$

50.17003 .8000 \#oxygen before $\mathrm{x}$-link

60.08003 .8000 \#CH ether

70.17003 .0700 \# oxygen bonded to ring

80.07003 .5500 \#carbon bonded to $\mathrm{O}$

90.17503 .9050 \#CH3

100.17003 .2500 \#non-xlink nitrogen

110.00000 .0000 \#hydrogen $x$-link

120.06603 .5000 \#carbon from $\mathrm{CH} 3$

130.03002 .5000 \#hydrogen from $\mathrm{CH} 3$

140.07003 .5500 \#graphene carbon 
150.11803 .8000 \#x-link CH2

160.17003 .8000 \#functional hydroxyl $\mathrm{O}$

170.00000 .0000 \#functional hydroxyl $\mathrm{H}$

180.17003 .8000 \#functional epoxide $\mathrm{O}$

190.17003 .2500 \#nitrogen 1-xlink

200.17003 .8000 \#crosslinked O

210.11803 .8000 \#crosslinked CH2

220.00000 .0000 \#crosslinked $\mathrm{H}$

230.17003 .2500 \#nitrogen 2-xlinks

Atoms

id molid type $\mathrm{x}$ y $\mathrm{z}$

11319.822945446396 .75103770114 .0837248078

21121.368965164796 .82157340854 .26562421574

31121.953016572598 .08918814344 .4079375841

.....FILE INCLUDES ALL ATOM POSITIONS, BONDS, ANGLES, AND

DIHEDRALS. 


\section{APPENDIX B}

\section{Copyright Agreements and Permissions for Figures}

\section{B.1. Permission to use Figure 1.2}

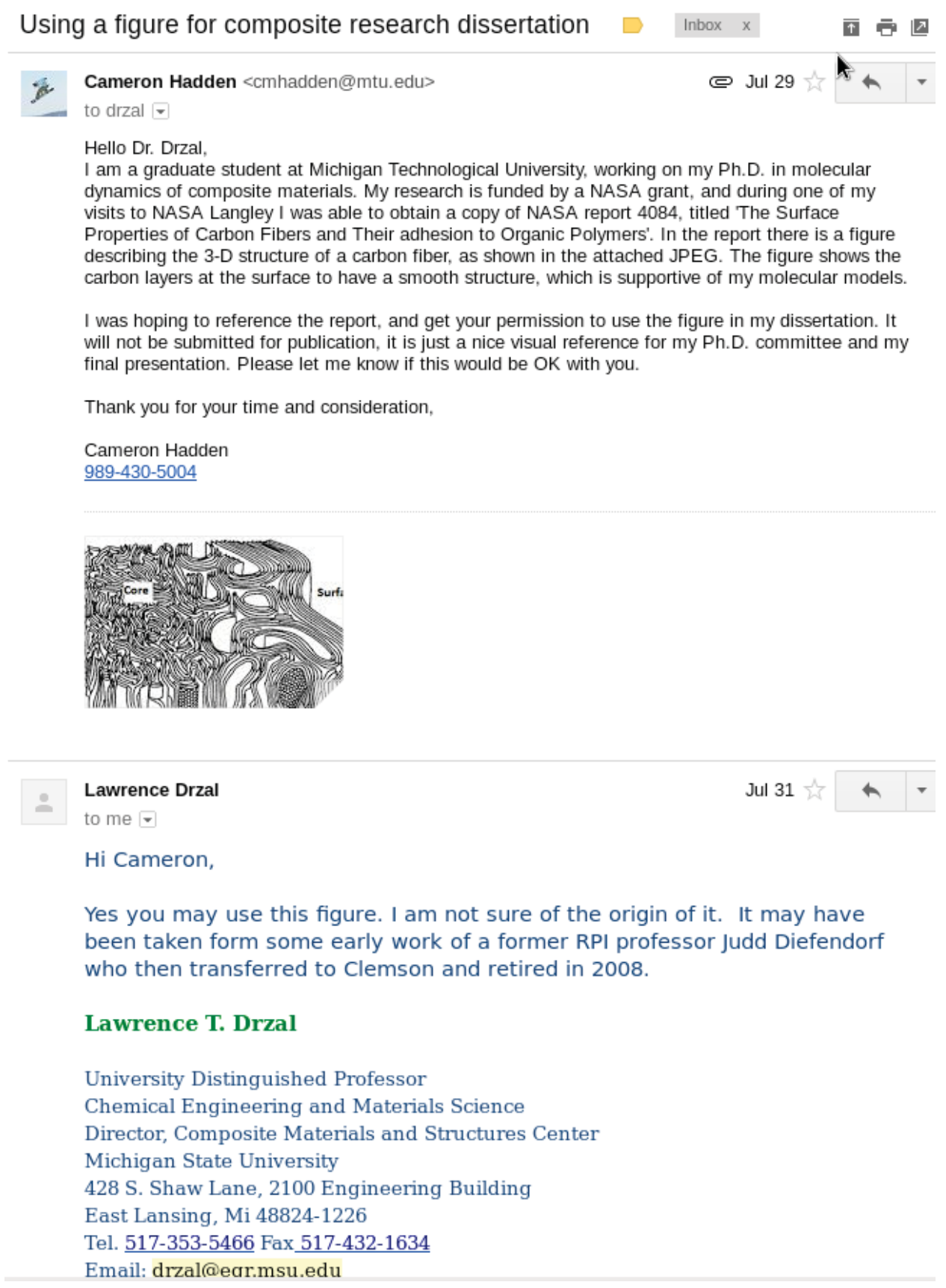




\section{B.2 Copyright Agreement for Text and Figures in Chapter 2}

ELSEVIER LICENSE

TERMS AND CONDITIONS

Aug 20, 2014

This is a License Agreement between Cameron M Hadden ("You") and Elsevier ("Elsevier") provided by Copyright Clearance Center ("CCC"). The license consists of your order details, the terms and conditions provided by Elsevier, and the payment terms and conditions.

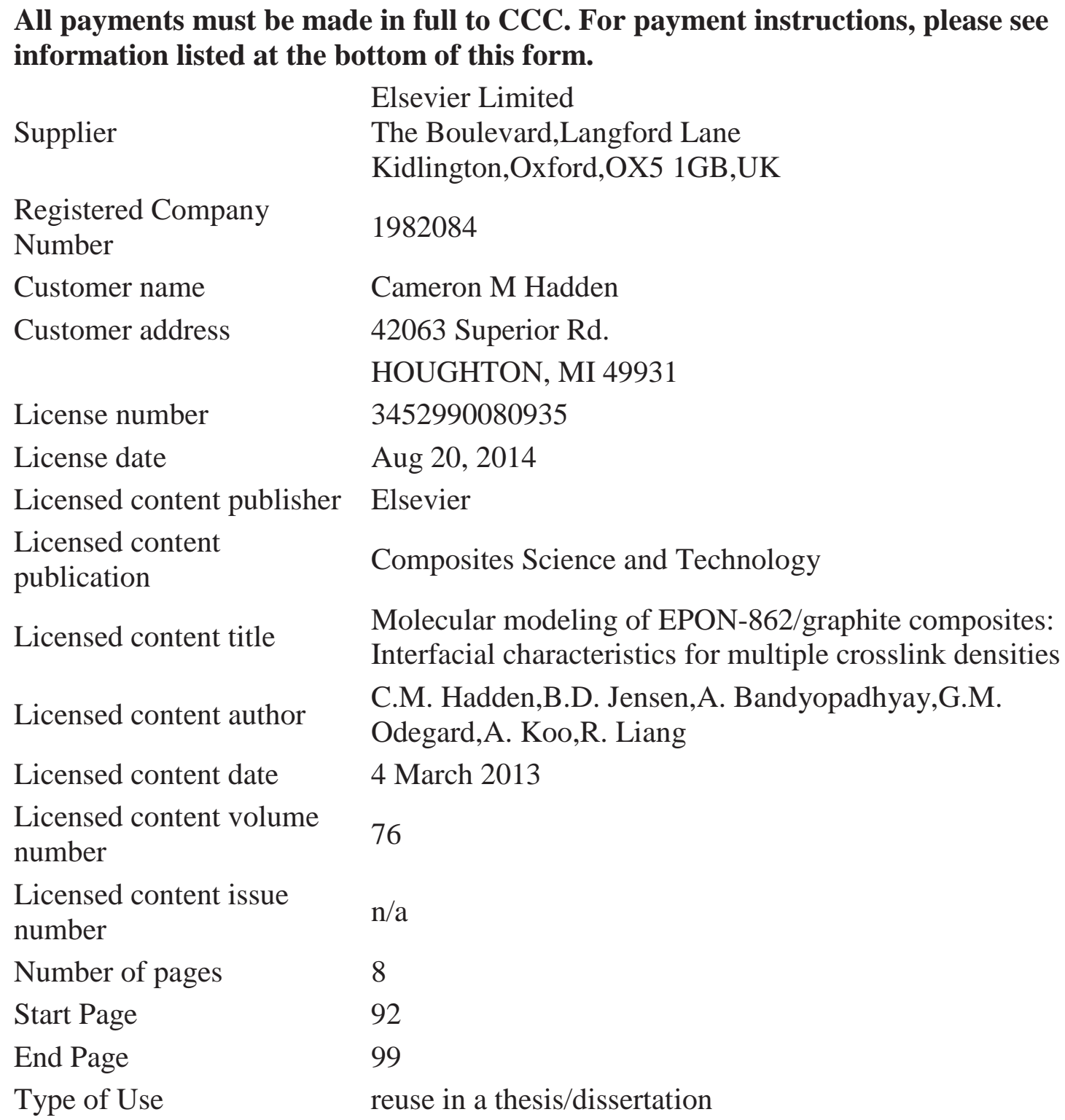


Portion

Format

Are you the author of this

Elsevier article?

Will you be translating?

Title of your

thesis/dissertation

Expected completion date

Estimated size (number of pages)

Elsevier VAT number

Permissions price

VAT/Local Sales Tax

Total

Terms and Conditions full article

both print and electronic

Yes

No

Molecular Modeling of EPON 862-DETDA / Carbon

Composites

Aug 2014

90

GB 494627212

0.00 USD

0.00 USD / 0.00 GBP

$0.00 \mathrm{USD}$ 


\section{INTRODUCTION}

1. The publisher for this copyrighted material is Elsevier. By clicking "accept" in connection with completing this licensing transaction, you agree that the following terms and conditions apply to this transaction (along with the Billing and Payment terms and conditions established by Copyright Clearance Center, Inc. ("CCC"), at the time that you opened your Rightslink account and that are available at any time at http://myaccount.copyright.com).

\section{GENERAL TERMS}

2. Elsevier hereby grants you permission to reproduce the aforementioned material subject to the terms and conditions indicated.

3. Acknowledgement: If any part of the material to be used (for example, figures) has appeared in our publication with credit or acknowledgement to another source, permission must also be sought from that source. If such permission is not obtained then that material may not be included in your publication/copies. Suitable acknowledgement to the source must be made, either as a footnote or in a reference list at the end of your publication, as follows:

"Reprinted from Publication title, Vol /edition number, Author(s), Title of article / title of chapter, Pages No., Copyright (Year), with permission from Elsevier [OR APPLICABLE SOCIETY COPYRIGHT OWNER].” Also Lancet special credit "Reprinted from The Lancet, Vol. number, Author(s), Title of article, Pages No., Copyright (Year), with permission from Elsevier."

4. Reproduction of this material is confined to the purpose and/or media for which permission is hereby given.

5. Altering/Modifying Material: Not Permitted. However figures and illustrations may be altered/adapted minimally to serve your work. Any other abbreviations, additions, deletions and/or any other alterations shall be made only with prior written authorization of Elsevier Ltd. (Please contact Elsevier at permissions @elsevier.com)

6. If the permission fee for the requested use of our material is waived in this instance, please be advised that your future requests for Elsevier materials may attract a fee.

7. Reservation of Rights: Publisher reserves all rights not specifically granted in the combination of (i) the license details provided by you and accepted in the course of this licensing transaction, (ii) these terms and conditions and (iii) CCC's Billing and Payment terms and conditions.

8. License Contingent Upon Payment: While you may exercise the rights licensed immediately upon issuance of the license at the end of the licensing process for the 
transaction, provided that you have disclosed complete and accurate details of your proposed use, no license is finally effective unless and until full payment is received from you (either by publisher or by CCC) as provided in CCC's Billing and Payment terms and conditions. If full payment is not received on a timely basis, then any license preliminarily granted shall be deemed automatically revoked and shall be void as if never granted. Further, in the event that you breach any of these terms and conditions or any of CCC's Billing and Payment terms and conditions, the license is automatically revoked and shall be void as if never granted. Use of materials as described in a revoked license, as well as any use of the materials beyond the scope of an unrevoked license, may constitute copyright infringement and publisher reserves the right to take any and all action to protect its copyright in the materials.

9. Warranties: Publisher makes no representations or warranties with respect to the licensed material.

10. Indemnity: You hereby indemnify and agree to hold harmless publisher and CCC, and their respective officers, directors, employees and agents, from and against any and all claims arising out of your use of the licensed material other than as specifically authorized pursuant to this license.

11. No Transfer of License: This license is personal to you and may not be sublicensed, assigned, or transferred by you to any other person without publisher's written permission.

12. No Amendment Except in Writing: This license may not be amended except in a writing signed by both parties (or, in the case of publisher, by CCC on publisher's behalf).

13. Objection to Contrary Terms: Publisher hereby objects to any terms contained in any purchase order, acknowledgment, check endorsement or other writing prepared by you, which terms are inconsistent with these terms and conditions or CCC's Billing and Payment terms and conditions. These terms and conditions, together with CCC's Billing and Payment terms and conditions (which are incorporated herein), comprise the entire agreement between you and publisher (and CCC) concerning this licensing transaction. In the event of any conflict between your obligations established by these terms and conditions and those established by CCC's Billing and Payment terms and conditions, these terms and conditions shall control.

14. Revocation: Elsevier or Copyright Clearance Center may deny the permissions described in this License at their sole discretion, for any reason or no reason, with a full refund payable to you. Notice of such denial will be made using the contact information provided by you. Failure to receive such notice will not alter or invalidate the denial. In no event will Elsevier or Copyright Clearance Center be responsible or liable for any costs, expenses or damage incurred by you as a result of a denial of your 
permission request, other than a refund of the amount(s) paid by you to Elsevier and/or Copyright Clearance Center for denied permissions.

\section{LIMITED LICENSE}

The following terms and conditions apply only to specific license types:

15. Translation: This permission is granted for non-exclusive world English rights only unless your license was granted for translation rights. If you licensed translation rights you may only translate this content into the languages you requested. A professional translator must perform all translations and reproduce the content word for word preserving the integrity of the article. If this license is to re-use 1 or 2 figures then permission is granted for non-exclusive world rights in all languages.

16. Posting licensed content on any Website: The following terms and conditions apply as follows: Licensing material from an Elsevier journal: All content posted to the web site must maintain the copyright information line on the bottom of each image; A hyper-text must be included to the Homepage of the journal from which you are licensing at http://www.sciencedirect.com/science/journal/xxxxx or the Elsevier homepage for books at http://www.elsevier.com; Central Storage: This license does not include permission for a scanned version of the material to be stored in a central repository such as that provided by Heron/XanEdu.

Licensing material from an Elsevier book: A hyper-text link must be included to the Elsevier homepage at http://www.elsevier.com . All content posted to the web site must maintain the copyright information line on the bottom of each image.

Posting licensed content on Electronic reserve: In addition to the above the following clauses are applicable: The web site must be password-protected and made available only to bona fide students registered on a relevant course. This permission is granted for 1 year only. You may obtain a new license for future website posting.

For journal authors: the following clauses are applicable in addition to the above: Permission granted is limited to the author accepted manuscript version* of your paper.

*Accepted Author Manuscript (AAM) Definition: An accepted author manuscript (AAM) is the author's version of the manuscript of an article that has been accepted for publication and which may include any author-incorporated changes suggested through the processes of submission processing, peer review, and editor-author communications. AAMs do not include other publisher value-added contributions such as copy-editing, formatting, technical enhancements and (if relevant) pagination.

You are not allowed to download and post the published journal article (whether PDF or HTML, proof or final version), nor may you scan the printed edition to create an 
electronic version. A hyper-text must be included to the Homepage of the journal from which you are licensing at http://www.sciencedirect.com/science/journal/xxxxx. As part of our normal production process, you will receive an e-mail notice when your article appears on Elsevier's online service ScienceDirect (www.sciencedirect.com). That e-mail will include the article's Digital Object Identifier (DOI). This number provides the electronic link to the published article and should be included in the posting of your personal version. We ask that you wait until you receive this e-mail and have the DOI to do any posting.

Posting to a repository: Authors may post their AAM immediately to their employer's institutional repository for internal use only and may make their manuscript publically available after the journal-specific embargo period has ended.

Please also refer to Elsevier's Article Posting Policy for further information.

18. For book authors the following clauses are applicable in addition to the above: Authors are permitted to place a brief summary of their work online only.. You are not allowed to download and post the published electronic version of your chapter, nor may you scan the printed edition to create an electronic version. Posting to a repository: Authors are permitted to post a summary of their chapter only in their institution's repository.

20. Thesis/Dissertation: If your license is for use in a thesis/dissertation your thesis may be submitted to your institution in either print or electronic form. Should your thesis be published commercially, please reapply for permission. These requirements include permission for the Library and Archives of Canada to supply single copies, on demand, of the complete thesis and include permission for UMI to supply single copies, on demand, of the complete thesis. Should your thesis be published commercially, please reapply for permission.

\section{$\underline{\text { Elsevier Open Access Terms and Conditions }}$}

Elsevier publishes Open Access articles in both its Open Access journals and via its Open Access articles option in subscription journals.

Authors publishing in an Open Access journal or who choose to make their article Open Access in an Elsevier subscription journal select one of the following Creative Commons user licenses, which define how a reader may reuse their work: Creative Commons Attribution License (CC BY), Creative Commons Attribution - Non Commercial - ShareAlike (CC BY NC SA) and Creative Commons Attribution - Non Commercial - No Derivatives (CC BY NC ND)

\section{Terms \& Conditions applicable to all Elsevier Open Access articles:}


Any reuse of the article must not represent the author as endorsing the adaptation of the article nor should the article be modified in such a way as to damage the author's honour or reputation.

The author(s) must be appropriately credited.

If any part of the material to be used (for example, figures) has appeared in our publication with credit or acknowledgement to another source it is the responsibility of the user to ensure their reuse complies with the terms and conditions determined by the rights holder.

\section{Additional Terms \& Conditions applicable to each Creative Commons user license:}

CC BY: You may distribute and copy the article, create extracts, abstracts, and other revised versions, adaptations or derivative works of or from an article (such as a translation), to include in a collective work (such as an anthology), to text or data mine the article, including for commercial purposes without permission from Elsevier

CC BY NC SA: For non-commercial purposes you may distribute and copy the article, create extracts, abstracts and other revised versions, adaptations or derivative works of or from an article (such as a translation), to include in a collective work (such as an anthology), to text and data mine the article and license new adaptations or creations under identical terms without permission from Elsevier

CC BY NC ND: For non-commercial purposes you may distribute and copy the article and include it in a collective work (such as an anthology), provided you do not alter or modify the article, without permission from Elsevier

Any commercial reuse of Open Access articles published with a CC BY NC SA or CC BY NC ND license requires permission from Elsevier and will be subject to a fee.

Commercial reuse includes:

Promotional purposes (advertising or marketing)

Commercial exploitation ( e.g. a product for sale or loan)

Systematic distribution (for a fee or free of charge)

Please refer to Elsevier's Open Access Policy for further information.

\section{Other Conditions:}

v1.6 
You will be invoiced within 48 hours of this transaction date. You may pay your invoice by credit card upon receipt of the invoice for this transaction. Please follow instructions provided at that time.

To pay for this transaction now; please remit a copy of this document along with your payment. Payment should be in the form of a check or money order referencing your account number and this invoice number RLNK501381820.

Make payments to "COPYRIGHT CLEARANCE CENTER" and send to:

\section{Copyright Clearance Center}

Dept 001

P.O. Box 843006

Boston, MA 02284-3006

Please disregard electronic and mailed copies if you remit payment in advance. Questions? customercare@ copyright.com or +1-855-239-3415 (toll free in the US) or +1-978-646-2777.

Gratis licenses (referencing \$0 in the Total field) are free. Please retain this printable license for your reference. No payment is required. 